듬 SCHOOL of GRADUATE STUDIES

EAST TENNESSEE STATE UNIVERSITY
East Tennessee State University Digital Commons@ East Tennessee State University

$8-2018$

\title{
Preparing for a National Weightlifting Championship: A Case Series
}

Spencer K. Travis

East Tennessee State University

Follow this and additional works at: https://dc.etsu.edu/etd

Part of the Sports Sciences Commons

\section{Recommended Citation}

Travis, Spencer K., "Preparing for a National Weightlifting Championship: A Case Series" (2018). Electronic Theses and Dissertations. Paper 3435. https://dc.etsu.edu/etd/3435

This Thesis - Open Access is brought to you for free and open access by the Student Works at Digital Commons @ East Tennessee State University. It has been accepted for inclusion in Electronic Theses and Dissertations by an authorized administrator of Digital Commons@ East Tennessee State

University. For more information, please contact digilib@etsu.edu. 
Preparing for a National Weightlifting Championship: A Case Series

\author{
A thesis \\ presented to \\ the faculty of the Department of Exercise and Sport Sciences \\ East Tennessee State University \\ In partial fulfillment \\ of the requirements for the degree \\ Master of Science in Applied Sport Science and Coach Education \\ by \\ Spencer Kyle Travis
}

August 2018

Caleb D. Bazyler, Ph.D., Chair

Satoshi Mizuguchi, Ph.D.

Michael H. Stone, Ph.D.

William A. Sands, Ph.D.

Keywords: Taper, Peaking, Weightlifting, Jump Height, Snatch, Clean and Jerk 


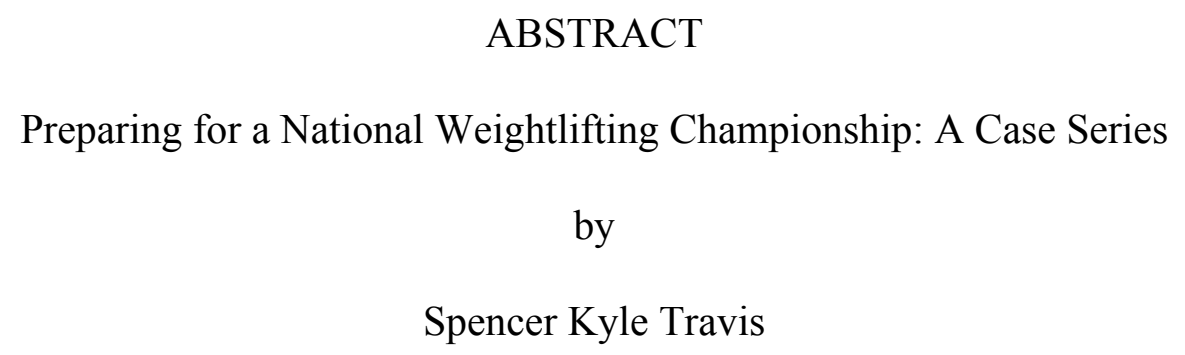

The purpose of this thesis was to observe psychological, physiological, and performance changes to determine when two high-level weightlifters were peaked for a major competition. We addressed this purpose by conducting a two-part case study series with one USA international level female and one USA national level male weightlifter. Both athletes were considered to be peaked on competition day. The results support our hypothesis that jumping performance would be peaked on competition day corresponding with an increased recovery and decreased stress state. However, contrary to our hypothesis, each athlete exhibited small decreases in muscle size leading into the competition relative to baseline values. Further, changes in inflammatory markers were inconsistent for each athlete and were not reduced as hypothesized. Based on our findings, this investigation supports the use of overreaching and tapering for individual strengthpower athletes providing insight into underlying psychological and physiological basis for observed changes in performance. 
Copyright 2018 by Spencer Kyle Travis

All Rights Reserved 


\section{DEDICATION}

I dedicate this thesis to my father and my grandfather. Both of you will be forever missed. Throughout the process of writing this manuscript, I never stopped whenever I was tired; I only stopped whenever I was done. Thank you both for teaching me the meaning of work, to never settle for good, and to leave my mark on this world before the Valkyries summon me home. 


\section{ACKNOWLEDGEMENTS}

I would like to thank the following people:

Dr. Caleb D. Bazyler for being the best scientific mentor I could have ever asked for and leading me down the path of righteous success.

Dr. Satoshi Mizuguchi for forever and always asking me 'why' and making me question my own existence.

Dr. Michael H. Stone for your insights and guidance throughout this process, as well as your pursuit for excellence in sport science research.

Dr. William A. Sands for giving me the most thorough insights and edits one could possibly receive and extending my library by a few hundred papers.

I wanted to put together one of the toughest committees I possibly could and I can say without hesitation that is indeed what I accomplished. Thank you all. 


\section{TABLE OF CONTENTS}

Page

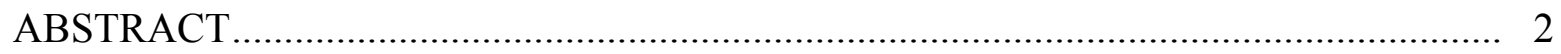

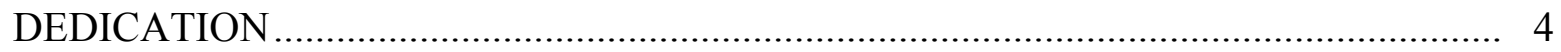

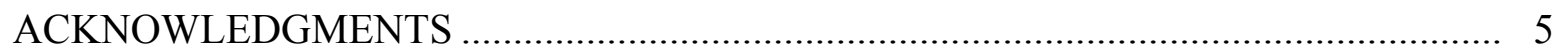

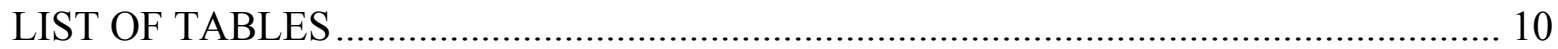

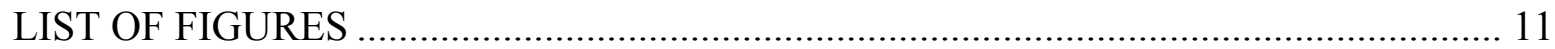

Chapter

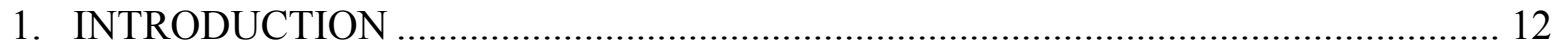

2. COMPREHENSIVE REVIEW OF THE LITERARURE........................................ 15

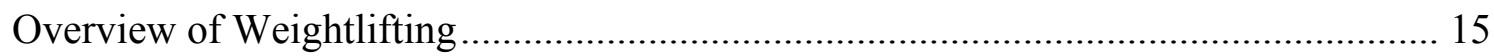

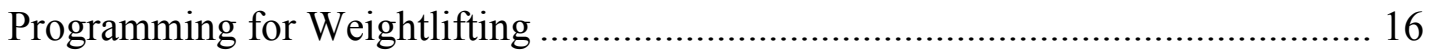

Current Practice and Training Principles ......................................................... 17

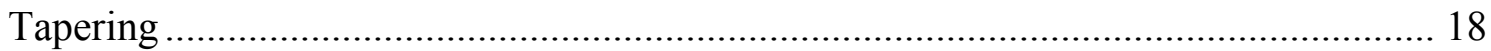

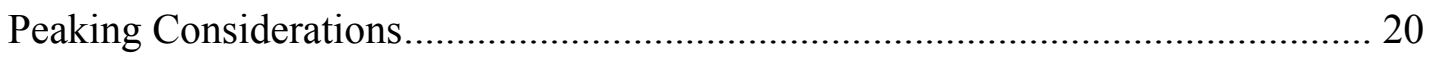

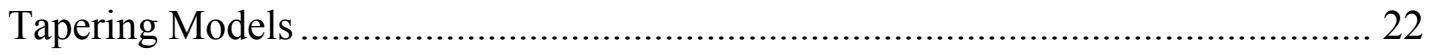

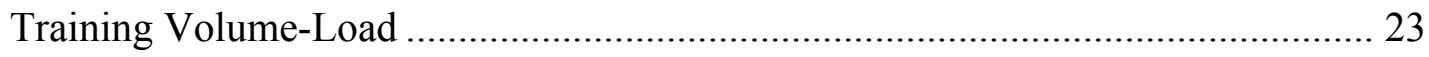

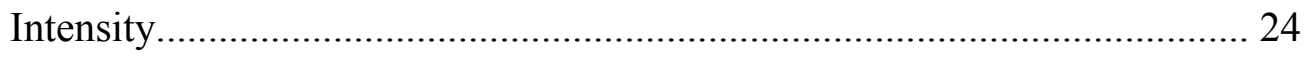

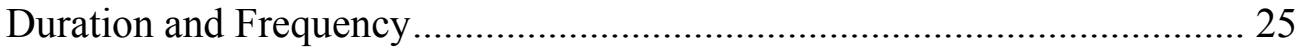

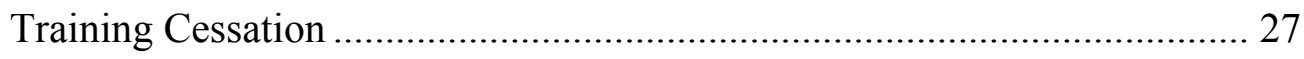

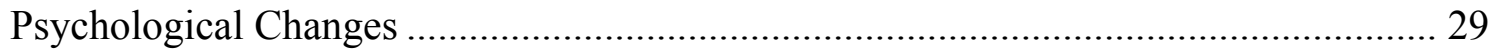




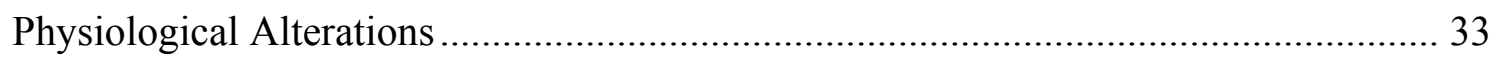

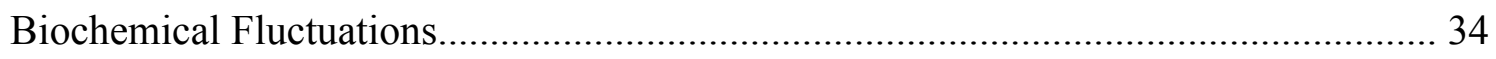

Endogenous Steroid Hormones........................................................................... 34

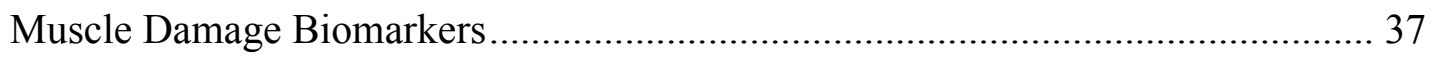

Considerations for the Female Athlete ………………………………………...... 39

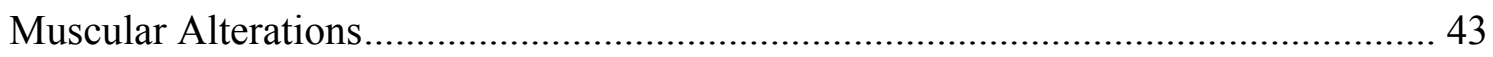

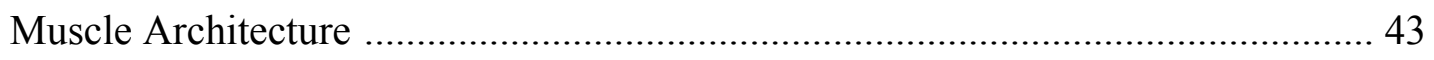

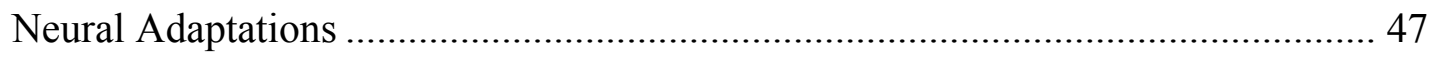

Performance Adaptation ....................................................................................... 51

Secondary Measure of Performance ……………………..................................... 52

Tapering Effects on Competition Performance ……………………....................... 53

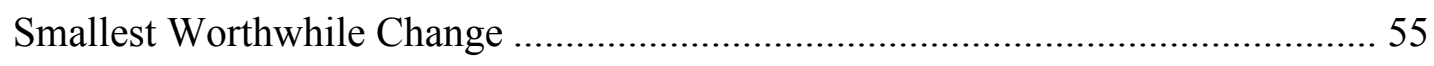

Tapering Practices for Weightlifting: A Brief Review ……………............................... 58

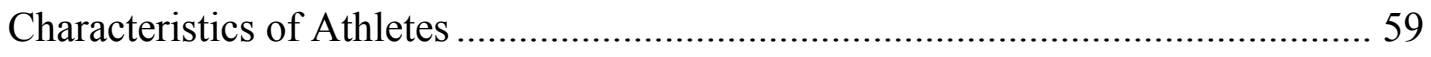

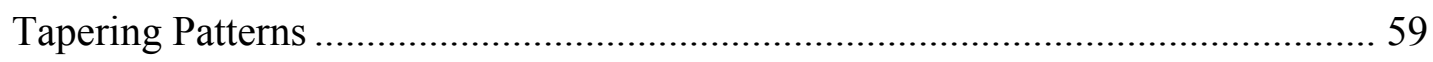

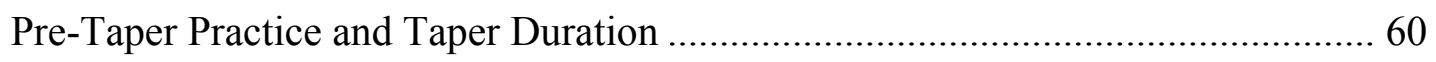

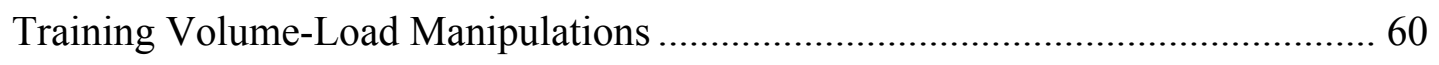

Performance Change Expectations ....................................................................... 61

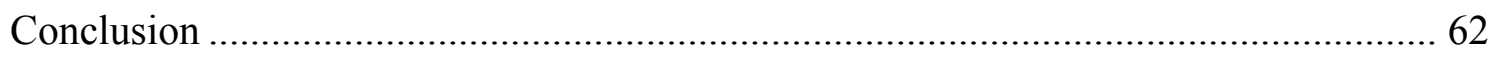

3. PREPARING FOR A NATIONAL WEIGHTLIFTING CHAMPIONSHIP: A CASE

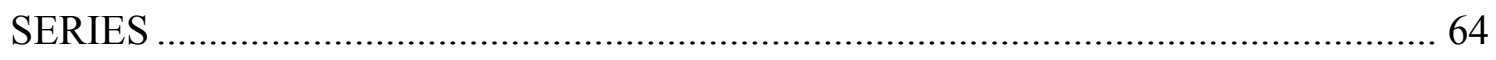

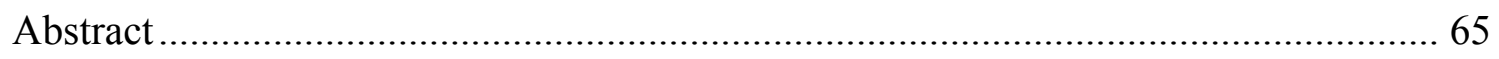

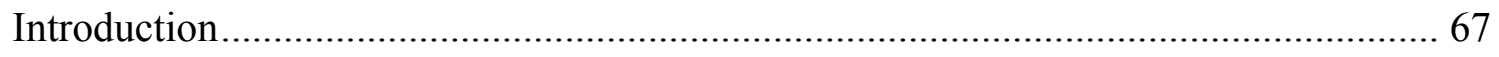


Methods.

Experimental Approach to the Problem.......................................................... 71

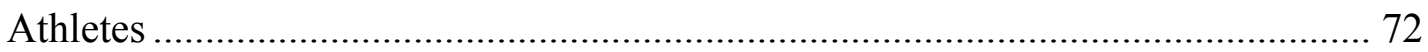

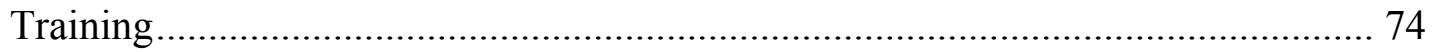

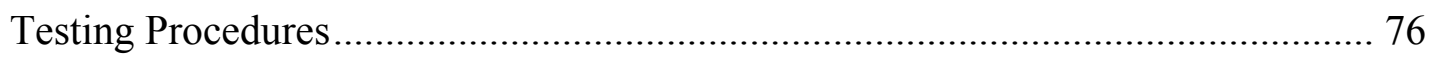

Anthropometrics and Hydration ....................................................... 76

Short Recovery and Stress Scale........................................................ 77

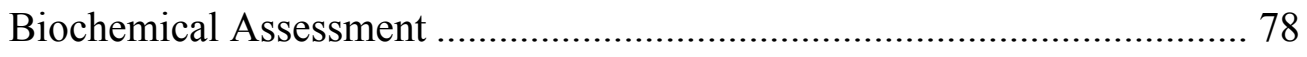

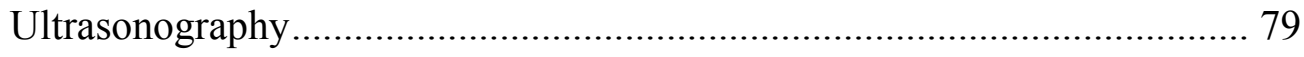

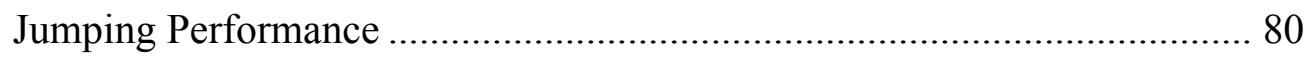

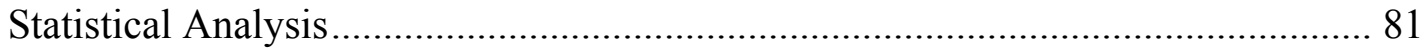

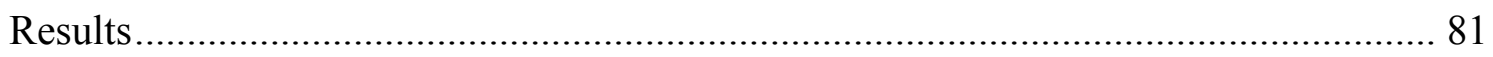

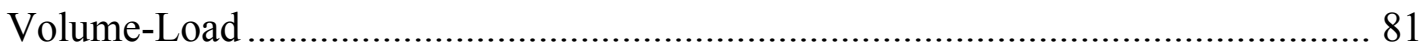

Weightlifting Performance, Anthropometrics, and Muscle Architecture ............... 83

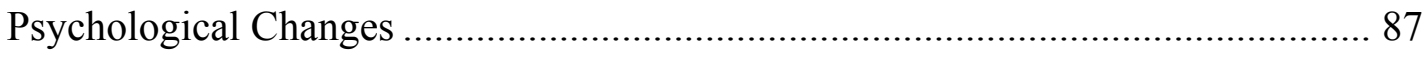

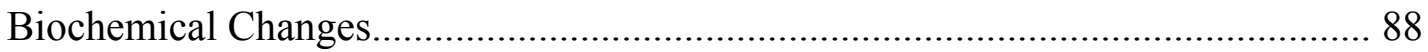

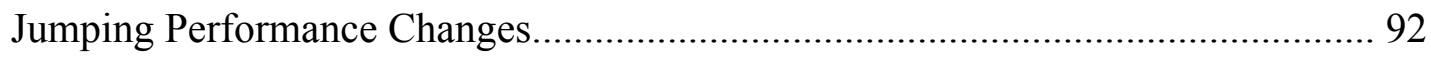

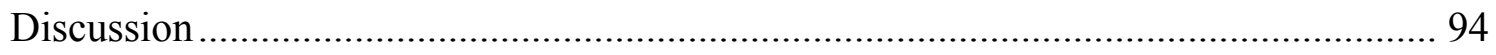

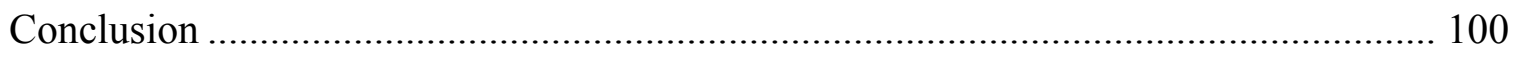

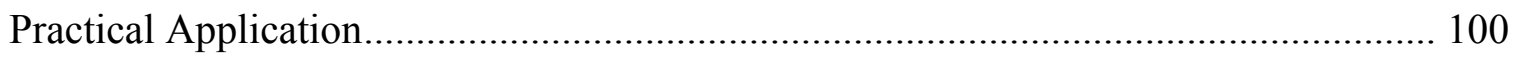

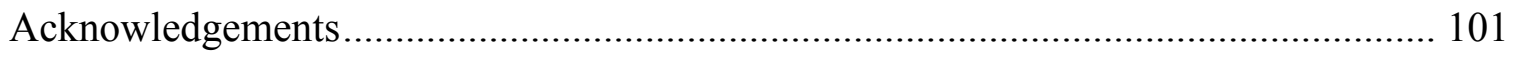

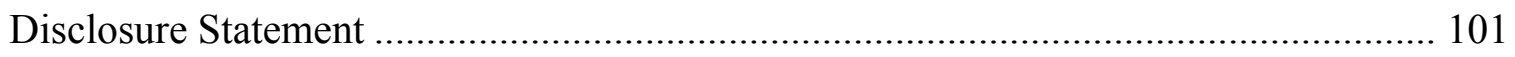

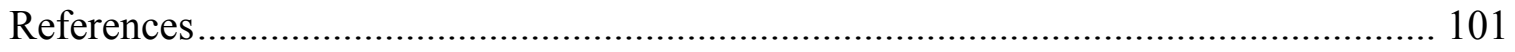


4. SUMMARY AND FUTURE INVESTIGATIONS ............................................. 110

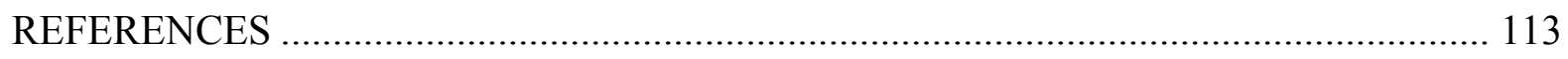

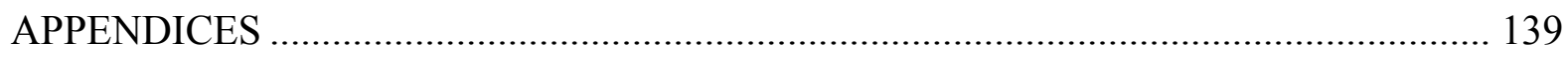

Appendix A: Potential Conflict of Interest Form.................................................. 139

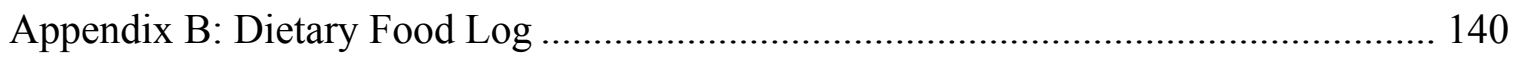

Appendix C: Sample of Short Recovery-Stress Scale ........................................... 142

Appendix D: Ultrasonography Imaging for Muscle Thickness ................................. 144

Appendix E: Ultrasonography Imaging for Muscle Cross-Sectional Area ................... 145

Appendix F: Sample Data of Squat Jump Performance Collection Program................ 146

Appendix G: Sample Data of Squat Jump Performance Analysis Program.................. 147

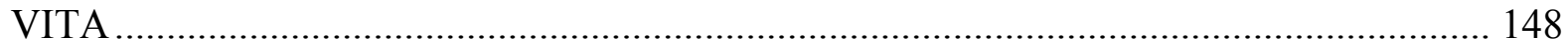




\section{LIST OF TABLES}

Table

Page

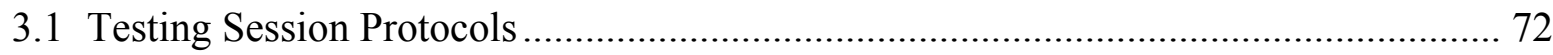

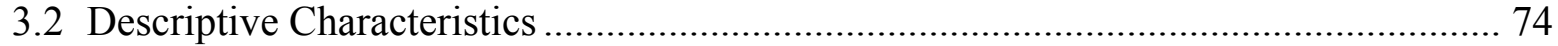

3.3 Final Training Block Leading into Competition for Case 1: Female Weightlifter....... 75

3.4 Final Training Block Leading into Competition for Case 2: Male Weightlifter ........... 76 


\section{LIST OF FIGURES}

Figure

Page

3.1 Training Volume Load during the Competition Preparation Phase 11 weeks out from competition date for the female weightlifter

3.2 Training Volume Load during the Competition Preparation Phase 11 weeks out from competition date for the male weightlifter 83

3.3 Body Mass changes during Competition Preparation Phase (female) 84

3.4 Muscle Thickness and Cross-Sectional Area changes during Competition Preparation Phase (female) 85

3.5 Body Mass changes during Competition Preparation Phase (male)..... 86

3.6 Muscle Thickness and Cross-Sectional Area changes during Competition Preparation Phase (male) 86

3.7 Short Recovery Stress Scale scores during Competition Preparation Phase (female).. 87

3.8 Short Recovery Stress Scale scores during Competition Preparation Phase (male) ..... 88

3.9 Endogenous Steroid Hormone changes during Competition Preparation Phase (female) 89

3.10 Inflammatory response during Competition Preparation Phase (female) 90

3.11 Endogenous Steroid Hormone changes during Competition Preparation Phase (male). 91

3.12 Inflammatory response during Competition Preparation Phase (male) 92

3.13 Jumping Performance during Competition Preparation Phase (female) 93

3.14 Jumping Performance during Competition Preparation Phase (male). 94 


\section{CHAPTER 1}

\section{INTRODUCTION}

Throughout the training process, it is vital to monitor psychological and physiological strain while subsequently enhancing recovery from training-induced stress and other stressors. External stressors outside of training are presumably erratic, therefore, understanding how to manipulate training-induced stress within the training macrocycle is vital to optimize performance. Manipulating stress at a specific point in time prior to a competition (COMP) is important when attempting to peak an athlete for a major COMP. Understanding how, when, and why to manage fatigue for high-level athletes is critical in order for the athlete to express a positive performance adaptation on the day of COMP (Halson, 2014). In order to achieve a positive performance gain, a peaking phase must be structured at the right time so accumulated fatigue can dissipate at an appropriate rate relative to the major COMP. Peaking high-level athletes (e.g., elite international, sub-elite - national level) at the appropriate time can be a difficult process causing acute psychological and physiological alterations while the sport scientists and coach's intent is to push the limits in training in order to perform at a higher level later on. Studies on high-level weightlifters have employed such peaking phases that include an overreach week (e.g., short term period of intensified training) with a subsequent taper (e.g., reduced training) where training volume is reduced, intensity maintained or increased, and largely frequency sustained prior to the major COMP (Bazyler et al., 2018; Busso, Häkkinen, Pakarinen, Kauhanen, Komi, Lacour, 1992; Mujika, 2009). Thus, knowing how and when to implement these principles appropriately may allow the athlete to peak so that preparedness can be expressed relative to the day of COMP. 
Success in the sport of weightlifting depends heavily upon leg and hip strength, which are important for the athlete to generate large ground reaction forces in a short time frame (Stoessel, Stone, Keith, Marple, Johnson, 1991). Training for weightlifting throughout the literature suggests that there is a high demand for incorporating the COMP lifts (i.e., snatch and clean and jerk) at high-intensities (i.e., $\geq 90 \%$ of 1-repetition maximum) repeatedly in order to mimic a COMP setting. However, an imbalance between stress and recovery could result in nonfunctional overreaching leading to maladaptation (Meeusen et al., 2013). Thus, providing a time period that promotes adequate recovery is required in order to achieve positive performance changes. The taper is a period of reduced training prior to the COMP that aims at achieving peak performance at the right time (Mujika \& Padilla, 2003). However, tapering is implemented after an appropriately applied overreach, which has been shown to aid in enhancing performance in weightlifters (Pistilli, Kaminsky, Totten, Miller, 2008; Stone \& Fry, 1998). The taper is the final necessary component for completing the training program prior to an important COMP.

An effective training program is constructed through a proper periodized plan that allows the right balance of volume, intense training, and recovery cycles to take place at appropriate times. Throughout the literature, proper COMP strategies within the training program that implement tapering has demonstrated performance improvements between 0.5 to $6.0 \%$ in high-level athletes but varies according to sporting demands and between each individual athlete (Mujika \& Padilla, 2003). Male and female weightlifters competing in the 2000 Olympic Games have shown performance variations for the COMP lifts and COMP totals averaging 2.5\% relative to the athlete's previous best lifts (McGuigan \& Kane, 2004). McGuigan and Kane (2004) suggests that the top 5 athletes in each weight class have an even narrower performance variation and the sport 
scientists and coach should aim at enhancing performance as little as $1.2 \%$ to ensure a top 5 finish. Mujika, Padilla, and Pyne (2002), suggest that by incorporating a tapering period the athlete is in an advantageous standing to receive a medal as opposed to an athlete not using a taper prior to competing. According to Mujika et al. (2002), the taper may also allow the sport scientist or coach to determine the level of the athlete (i.e., highly-trained, moderately trained, intermediate), and which tapering method should be used for future COMP. Unfortunately, tapering practices in elite sports for strength-power athletes are almost exclusively based on the coaches experience with little to no input from sport scientists (Mujika et al., 2002).

Although several studies have established optimal tapering strategies for endurance sport athletes (Mujika, 2009) there is still a paucity of tapering literature for strength-power athletes. Therefore, the purpose of this thesis was to characterize the psychological, physiological and performance changes of two high-level weightlifters preparing for a major national championship. The primary focus was to determine when peak jumping performance was achieved and whether psychological or physiological variables could explain any jumping performance changes. The investigators examined changes during the athlete's training preceding, the day of, and following the COMP to determine when peak jumping performance was achieved. We expected jumping performance to be peaked the day of COMP corresponding with improved recovery-stress scores, reduced serum inflammatory markers, and preserved muscle size relative to baseline values. 


\section{CHAPTER 2}

\section{COMPREHENSIVE REVIEW OF THE LITERATURE}

A well-constructed program for any high-level athlete is aimed at reaching a peak and attaining the highest performance level possible at a major competition (COMP) within the training macrocycle. Throughout the training process, the athlete must adapt to new training stimuli in order to drive positive performance gain later on. By properly planning successive and sequential training blocks at appropriate times, the sport scientist and coach increase the athlete's chances of success. It is likely that positive performance gain will be expressed if and when psychological and physiological variables are in synergy that will directly influence performance. Often, this synergy is accomplished by applying an overreaching stimulus followed by a strategic tapered training period prior to the COMP.

Unfortunately, the current literature is limited to predominately endurance athletes. Thus, there is a paucity of literature dealing with proper peaking and tapering strategies for strengthpower athletes such as weightlifters. Therefore, the purposes of this review are to define and characterize tapering, examine psychological and physiological changes that may explain performance changes from tapering, and outline performance changes, specifically for weightlifters, using a taper after an overreach during a COMP period.

\section{Overview of Weightlifting}

Weight lifting, weightlifting, or Olympic weightlifting? These terms are often used interchangeably; however, by the conclusion of this section, the reader will be able to differentiate between each term, respectively. Weight lifting is the act of an individual or an 
athlete lifting weights which are also referenced to as resistance training (Stone, Pierce, Sands, \& Stone, 2006). Weightlifting is a strength-power sport where two lifts are performed with a barbell contested amongst men and women (Stone, Pierce, et al., 2006). Olympic weightlifting should be referred to properly as the sport of weightlifting being contested at the Olympic games (Stone, Pierce, et al., 2006). In the sport of weightlifting, male and female athletes in their respective weight categories contest two lifts: the snatch and the clean and jerk $(\mathrm{C} \& \mathrm{~J})$. The snatch is a single movement where the lifter takes the bar from the floor overhead in one single motion. The C\&J is a two-part movement where the lifter pulls the bar from the floor to the shoulders (i.e., clean). The bar is then moved from the shoulder position to the overhead position by the lifter bending the knees and driving the bar upward and catching the weight overhead (i.e., jerk). Each athlete is given three attempts beginning with the snatch followed with the C\&J. Weightlifting is divided into weight classes, with the highest winning each class. The heaviest successful attempt (i.e., quantified in kilograms [kg]) for each lift are then summed together in order to determine the best lifter. Each lifter's weightlifting total is then adjusted accordingly to the polynomial Sinclair formula which gives each athlete a Sinclair total (Stone, Pierce, et al., 2006). Theoretically, the heaviest athlete should have the heaviest total and would win the COMP (Beckham et al., 2013). However, the Sinclair total largely obviates differences in body mass for males and females, respectively, and identifies the best overall lifter by scaling body mass to the actual performance of the total mass lifted (Hornsby et al., 2017).

\section{Programming for Weightlifting}

Training programs for weightlifters are designed with a high degree of specificity for the snatch and C\&J. However, there is very limited evidence comparing training adaptations with 
performance outcomes due to the high variance in program styles depending on the origin of countries who participate in weightlifting as well as the number of weightlifters available for such research (Drechsler, 1998; Garhammer \& Takano, 2003; González-Badillo, Izquierdo, \& Gorostiaga, 2006; Stone, Pierce E, Sands, \& Stone, 2006; Storey \& Smith, 2012). In the 1970s, the Soviet Union and Eastern Bloc lifters (i.e., Bulgarians) were extremely successful in the sport of weightlifting, which has been attributed to steroid use (Storey \& Smith, 2012). Popular weightlifting programs in recent years have stemmed from the notorious Soviet Union and Eastern Bloc lifters from the 1970s despite the programs designed to encompass known usage of anabolic steroids (Fair, 1988; Franke \& Berendonk, 1997; Stone, Pierce, et al., 2006). In general, overreaching, overtraining, and fatigue are often closely monitored in weightlifting programs, especially at the highest level (Fry et al., 1994; Pistilli et al., 2008; Stone, Pierce, et al., 2006). In order to ensure adequate recovery and reductions in training stress for non-anabolic using weightlifters, Western coaches have to make significant modifications to the training programs (Stone, Pierce, et al., 2006). Most weightlifting programs divide the daily volume into two training sessions to ensure maximal adaptations (Häkkinen \& Kallinen, 1994; Hartman, Clark, Bembens, Kilgore, \& Bemben, 2007). It has been suggested that dividing volume into multiple training sessions allows faster recovery thus, improving training and COMP results.

\section{Current Practice and Training Principles}

In a recent review (Storey \& Smith, 2012), the authors point out some of the similarities and differences between the former Soviet Union, Bulgarians, and modern-day training methods. Although each of these methods varies greatly between preparatory phases and COMP phases, each method incorporated adjustments in training intensity and total volume load prior to a 
COMP (Stone, Pierce E, et al., 2006; Takano, 1989; Zatsiorsky, 1992). Historically, weightlifting training programs include 1-3 weeks of increased loading (i.e., overreach) followed by 1-3 weeks of reduced loading (i.e., taper) prior to the COMP (Pistilli et al., 2008). Within the body of literature, appropriately applying overreach periods prior to COMP is suggested to be a major contributory factor that improves weightlifting performance, espically for sequential COMP throughout the macrocycle (Bazyler et al., 2018; T. Busso, Häkkinen, et al., 1990, 1992; Fry, Kraemer, Stone et al., 1994; Fry \& Kraemer, 1997; Fry, Kraemer, Stone et al. 2000; Fry et al., 2006; González-Badillo, Izquierdo, Gorostiaga, 2006; Haff et al., 2005, 2008; Häkkinen, Komi, \& Kauhanen, 1986; Häkkinen, Pakarinen, Alén, Kauhanen, \& Komi, 1987; Hornsby et al., 2017; Kraemer et al., 1992; Pistilli et al., 2008; Stoessel et al., 1991; Stone \& Fry, 1998; Stone et al., 1996; Wu, Hung, Wang, \& Chang, 2008). However, it is important to note the majority of these studies primarily focus on the overreaching stimulus and pay little to no attention to the tapering period thereafter. Only a few of these studies have focused, to some degree, on post-overreach tapering effects psychologically, physiologically, or on performance changes in weightlifters (Bazyler et al., 2018; Hornsby et al., 2017; Stone \& Fry, 1998; Stone et al., 1996; Wu et al., 2008).

\section{Tapering}

In weightlifting, as well as in other strength-power sports, high-level athletes competing at the national and international level undergo rigorous training protocols attempting to peak for a major COMP. However, regardless of COMP level, every athlete has a baseline performance level that is indicative of the athlete's general fitness characteristics (Chiu \& Barnes, 2003). Whenever a high training stimulus is applied, the athlete's response typically results in a 
performance decrement simultaneously diminishing preparedness as explained by the fitnessfatigue paradigm (Thierry Busso, Candau, \& Lacour, 1994). However, depending on the magnitude and duration of the training stimulus, physiological and psychological disturbances from accumulated fatigue can remain elevated until the training stimulus is reduced or removed. In order to reduce accumulated fatigue which will, theoretically, unmask preparedness, rigorous training must be coupled with a reduced training period so that negative training effects dissipate. Thus, to negate fatigue tapering is used as a training strategy that reduces the training volume-load (VL). Using the theoretical infrastructure of the fitness-fatigue paradigm, reducing the training VL at the appropriate time and rate expounds on the relationship between applying a stimulus and receiving the resulting response (Chiu \& Barnes, 2003).

In sports such as rugby, soccer, and tennis, total training load is characterized by not only the training volume from resistance training but also practices, conditioning sessions, scrimmaging, matches, etc. With sports like weightlifting, as well as powerlifting or strongman, using a loaded barbell is part of training, practice, and COMP, therefore, training load and VL can be used interchangeably. For the sake of this review, the appropriate and accurate terminology to use will be $\mathrm{VL}$ which refers to the overall estimation of work being done by the athlete. A reduction in VL is typically implemented into the program immediately after a deliberate high-intensity, high-stress acute stimulus referred to as an overreach.

The purpose of a taper encompasses the underlying training methodology by manipulating training variables used to prescribe training in the final weeks leading up to the COMP (Pritchard, Keogh, Barnes, \& McGuigan, 2015). A taper can be defined as a progressive linear or 
non-linear reduction in VL during a variable period of time, to reduce the physiological and psychological stress of daily training to optimize performance (Mujika, 2010; Mujika, 2009). In a recent review by Murach and Bagley (2015), these authors have expressed concern that literature dealing with tapering is primarily based on endurance sport athletes and not so much strength-power athletes. Still, tapering is one of the most overlooked and misunderstood components of the training process when trying to reach a level of peak performance for a COMP. Even within the current literature, a caveat to using tapers for strength-power athletes is that most tapering designs tend to follow tapering parameters that have been established for endurance sport athletes (Pritchard et al., 2015; Pyne, Mujika, \& Reilly, 2009).

For example, in the recent review by Pritchard et al., (2015), it is recommended that tapering for maximal strength should consist of a taper duration lasting 1 to 4 weeks. This review also suggested reducing VL by $30-70 \%$ with maintaining or slightly reducing training frequency to attain VL reductions. However, also vitally important for tapering in endurance sports, maintaining or slightly increasing training intensity relative to the overreach is also recommended. Consequently, using the aforementioned recommendations could potentially prohibit strength-power athletes from expressing true levels of peak performance on COMP day. Thus, a more thorough understanding of how, when, and why to implement the taper after the overreach for strength-power athletes is needed.

\section{Peaking Considerations}

The overreach can be defined as an acute accumulation of training and/or non-training stress resulting in short-term decrements in performance stemming from a deliberate high volume, 
high-stress training environment (Pistilli et al., 2008; Stone et al., 1996). Training success in high-level athletes is often attributed to a planned 1-2 week overreach period prior to a COMP. However, if an excessive overreach is applied resulting in failure to recover then a negative performance outcome is likely. The recent position statement from the American College of Sports Medicine and the European College of Sport Science states that planned overreaching is not functional overreaching unless it results in performance supercompensation during a subsequent recovery period (Meeusen et al., 2013). However, current literature still suggests that there is a significant difference between a non-functional overreach and a functional overreach prior to COMP and may be planned accordingly (DeWeese, Hornsby, Stone, \& Stone, 2015a, 2015b). While a planned functional overreach promotes performance gain, a non-functional overreach is a type of involution that occurs resulting in maladaptation from accumulated fatigue (DeWeese et al., 2015a). This is a state where the athlete is still experiencing negative training effects with significant psychological and/or physiological disruptions.

However, a timeframe when the athlete experiences a rebound effect (i.e., supercompensation) from overreaching allows acute performance improvements to be expressed assuming that the athlete is fully recovered following the reduced training period. (Stone, O'Bryant, \& Garhammer, 1981). Due to the lack of evidence for supercompensation, the overreach should not be referred to as functional or non-functional during the training process until the subsequent performance outcome is determined and expressed in a positive or negative manner. Thus, planned overreaching would be more appropriate being functional or nonfunctional overreaching could take place with or without psychological and physiological signs and symptoms related to negative or positive performance trends that may take days or weeks for 
the athlete to recover from. Planned overreaching for a less important COMP using different tapering models may be needed in order for the sport scientist and coach to assess how the athlete responds to various manipulations in VL.

\section{$\underline{\text { Tapering Models }}$}

Several patterns of tapering have been thoroughly investigated to establish an optimal tapering model to improve performance repeatedly. Currently, there are four tapering models that are used for athletes when preparing for COMP. The four tapering models that have been outlined in the literature include the linear taper, step-taper, and two exponential tapers involving a fast or slow constant decay (i.e., reduction) in VL (Mujika, 2009; Pyne, Mujika, \& Reilly, 2009). The linear taper is considered to be a theoretical reference but does not actually exist in practice due to too many undulations in training duration, frequency, and intensity. A step-taper involves a sudden reduction (e.g., 50\%) in VL by a constant amount as opposed to the more progressive tapering models where gradual manipulation in VL can be used. An exponential taper can contain a fast constant decay (i.e., sudden reduction every 4 days) or a slow constant decay (i.e., gradual reduction every 8 days) in the VL (Mujika, 2009). The fast decaying exponential taper, theoretically, accelerates recoverability from accumulated fatigue at the same rate as reductions in VL compared to using a slow decay (Banister, Carter, \& Zarkadas, 1999). Monitoring psychological, physiological and performance changes across different taper models by manipulating VL in various ways can give insight into how to taper the athlete appropriately. 


\section{$\underline{\text { Training Volume-Load }}$}

Each one of the aforementioned tapering models utilizes a reduction in VL relative to the amount of work the athlete is completing. Specifically, for weightlifting, a simple and practical way to calculate VL can be described by this formula:

$$
V L=\text { load lifted }(\mathrm{kg}) \times \text { number of reps } \times \text { number of sets }
$$

For a weightlifter, all warm-ups, working sets, and down-sets, if programmed accordingly should be documented to accurately represent VL. This information should be recorded for all training sessions to gauge the effectiveness of the program for each athlete, respectively and to quantify the amount of overall work accomplished. Theoretically, by accurately calculating normal VL, reductions in VL during the taper will allow for adequate recovery to take place from previous successive training sessions so performance adaptations can be expressed later on (Bosquet, Montpetit, Arvisais, \& Mujika, 2007; Pritchard et al., 2015; Pyne et al., 2009). Calculating VL as described is the most practical and time efficient means when accounting for the amount of work an athlete is doing. However, although it is not necessary, more robust methods of calculating VL with displacement using this formula:

$$
V L w D=V L x \text { displacement }(\mathrm{cm})
$$

should be used when possible to get a more accurate estimation of total work being accomplished (Hornsby, 2013).

The taper is the product of VL manipulations that includes all variables (i.e., training intensity, duration, and frequency) in a systematic and progressive or gradual manner as opposed to just a reduction in one variable or the other (Houmard, 1991). For weightlifters and other strength-power athletes, Pritchard et al., (2016) note that the most effective taper shown to 
increase maximal strength has been with a step taper or fast decaying exponential taper. Thus, an effective taper for strength-power athletes requires the VL to typically reduce by approximately $30-70 \%$ of the pre-taper VL (i.e., overreach) amount while keeping the training intensity high using one of the two the tapering models (Murach \& Bagley, 2015). The aim of the taper is to reduce the negative physiological and psychological impact of daily training (i.e., accumulated fatigue) rather than achieving further improvements with positive adaptions from training (i.e., fitness gains) (Mujika, 2010). Therefore, this reduction in VL is necessary for promoting adequate recovery so that the athlete can perform at a higher level relative to the day of COMP. However, manipulating training variables to reduce VL should be planned appropriately with emphasis on maintaining training intensity being a reduction in intensity could be detrimental for training-induced adaptations (Mujika, 2010) and the exercise selection may become more specific to the COMP lifts.

Intensity. Training intensity can be defined by the percentage of maximal performance an athlete gives or is prescribed during normal training periods relative to the athlete's best performance. Mujika (2010) suggest that maintaining training intensity throughout the taper is vital in order to express positive training adaptations during COMP. The review by Pritchard et al. (2016) also suggests that maintaining or increasing training intensity with reductions in VL has greater benefits compared to reducing training intensity and VL together. For example, a recent tapering study using track and field throwers examined the effects of a 2-week taper manipulating intensity with heavy versus light loading (Zaras et al., 2014). The throwers who used higher training intensities ( $>85 \%$ 1-repetition maximum (RM)) during the taper resulted in greater performance improvements on leg press 1RM, rate of force development (RFD), vertical 
jump power output, and shot throw distance compared to the throwers tapering with lower training intensities (30\% 1RM). Therefore, the pre-taper intensity used during the overreach should be at least maintained or slightly increased during the reduced training period for strength-power athletes so fitness gains are retained (Pistilli et al., 2008). Maintaining or slightly increasing intensity will also allow the athlete to emulate the intensity needed at the COMP which is important for sports such as weightlifting where efforts at or above $100 \%$ are required. The athlete's ability to recover is a product of VL manipulations (i.e., total volume, training frequency, training duration) that should not be reduced at the cost of decreasing training intensity (Mujika, 2010). Thus, if the training intensity is reduced or removed (i.e., training cessation) too soon this could lead to a loss of newly gained adaptations from a lack of training stimulus (Mujika, 2010). Therefore, according to Mujika (2010), arguably, training intensity is key to eliciting a positive performance change throughout the duration of the taper with an emphasis on mimicking COMP performance (i.e., snatch and C\&J).

Duration and Frequency. Based on the majority of tapering literature dealing with endurance sport activities, tapering periods of 8-14 days appear to be the optimal timeframe to elicit a positive performance outcome (Bosquet et al., 2007) whereas tapering strategies lasting from 414 days (Mujika \& Padilla, 2003) have also shown to induce enhanced performance, psychological, and physiological states. It has also been suggested that male athletes may require longer tapering periods than female athletes due to factors such as overall VL differences, hormone changes, and stress coping factors (Mujika, 2009). Specifically for weightlifters, short and long taper lengths have shown to be effective for elite males preparing for a national championship (Stone et al., 1996). In this study, a group of 5 weightlifters used a short taper 
lasting 7 days and the second group of 5 used a long taper lasting 28 days. A positive performance outcome was observed in both groups eliciting a COMP total increase of $8 \mathrm{~kg}$ and $17.5 \mathrm{~kg}$, respectively. Although the long taper duration showed more improvement, taper durations for weightlifters lasting 7-28 days still agree with the current recommendations based on endurance training that range from 1-4 weeks (Mujika, 2009). However, observing when the highest intensities are prescribed during the reduced VL for each taper length may give more insight into which duration is most effective relative to the intensity being used. This could also be indicative of how training frequency should be adjusted for strength-power athletes so that positive performance levels are expressed at a peaked state.

Coinciding with the idea of manipulating VL and intensity over the right duration, training frequency is also an important factor when considering training duration. Training frequency during the taper can be described by the total number of training sessions that take place during the duration of the taper timeline (Wilson \& Wilson, 2008). It has been suggested that training frequency can be reduced up to $20 \%$ during the taper in order to realize performance improvements in high-level aerobic athletes (Mujika, Padilla, \& Pyne, 2002). However, decreasing training frequency $>20 \%$ could lead to minimal performance gain, performance decrements, or no change in performance at all. This suggests that training frequency should not be substantially manipulated as an alternative method for reducing VL, especially for sports such as weightlifting that is technique oriented which requires the athlete to practice the COMP movements frequently. Therefore, at the end of a taper, the duration of training cessation prior to the COMP should be planned carefully to prevent loss of adaptation considering that training frequency will be inevitably reduced when traveling to a COMP. 
Training Cessation. Although manipulating training frequency is not suggested, an acute period of complete rest (i.e., cessation from training) in the final stages of the taper are important to ensure the athlete is recovered and prepared to compete on COMP day. Aerobic athletes can sustain maximum performance levels with training cessation lasting from 10 days up to 5 weeks (Mujika \& Padilla, 2000), but this is likely due to aerobic adaptations decaying at a slower rate than strength-power adaptations once the training stimulus is completely removed (Mujika \& Padilla, 2000). For example, in strength trained males, during 4 weeks of training cessation 1RM back squat, bench press, and half squat declined by $10 \%, 9 \%$, and 6\%, respectively (Izquierdo et al., 2007). In elite level male kayakers, training cessation over a 5-week period resulted in markedly large reductions in muscle power and maximal strength (García-Pallarés, SánchezMedina, Pérez, Izquierdo-Gabarren, \& Izquierdo, 2010). Based on these findings, it can be speculated that maximal muscle power levels are lost more rapidly than maximal strength in high-level athletes, which are two important fitness characteristics to perform optimally for weightlifting.

Beginning an acute period of training cessation too early (i.e., $>1$ week), or too late (i.e., $<24$ hours before COMP) for strength-power athletes, could cause negative psychological and physiological changes resulting in negative performance outcome. It has been recently suggested that more efficient neuromuscular function (i.e., less neuromuscular fatigue) allows for better expression of maximal strength as shown in strength athletes with training cessation lasting between 2 days up to 1 week and optimal performance expressed during 4 days of complete training cessation (Pritchard, Barnes, Stewart, Keogh, \& McGuigan, 2018). However, with the tapering strategy aimed at balancing recovery and stress at the right time allowing training 
cessation to take place accordingly days prior to COMP may help the athlete supercompensate so positive performance changes are expressed relative to the COMP. For strength-power athletes, training may cease at least 2 days before the targeted COMP but no more than 1-week prior to the COMP (Pritchard et al., 2018).

Tapers are widely used by athletes from various sports to gain a performance edge over their competitors. It is evident that there is a trend of reducing VL, increasing or maintaining intensity, and a giving the athlete a brief cessation from training before COMP throughout the literature (Aubry, Hausswirth, Louis, Coutts, \& LE Meur, 2014; Banister, Carter, \& Zarkadas, 1999; Bosquet et al., 2007; Brännström, Rova, \& Yu, 2013; Busso et al., 1990, 1992; Busso et al., 1994; Coutts \& Murphy, 2007; Fry et al., 1994; Fullagar, McCunn, \& Murray, 2017; Gleeson et al., 2000; Häkkinen et al., 1987; Halson et al., 2002; Izquierdo et al., 2007; Kellmann \& Günther, 2000; Mujika, 2010; Mujika, Chatard, \& Geyssant, 1996; Mujika et al., 2002b; Mujika et al., 2000; Mujika \& Padilla, 2000, 2003b; Murach et al., 2014; Neary, Martin, \& Quinney, 2003; Painter et al., 2012; Pistilli et al., 2008; Pritchard et al., 2016; Pritchard et al., 2015; Pyne et al., 2009; Santos \& Janeira, 2009; Tønnessen et al., 2014; Trinity, Pahnke, Sterkel, \& Coyle, 2008; Trinity, Pahnke, Reese, \& Coyle, 2006; Vacher, Nicolas, Martinent, \& Mourot, 2017; Wilson \& Wilson, 2008; Wu et al., 2008; Zaras et al., 2014). A deep knowledge and understanding of tapering models and how to manipulate training variables are necessary for optimizing peak performance, especially when important COMP are contiguous in sports such as weightlifting. The key to a successful taper is to reduce fatigue so that adaptations can be expressed but to also avoid detraining ( partial or complete loss of training-induced physiological adaptations due to decreased of total VL) (Myers, McKillop, Fraser, Abel, \& Wells, 2017). By using the parameters 
for tapering that have been outlined in this review, acute training cessation at the end of the taper may not only give the athlete a physiological break from training but also a psychological break in order to prepare for the COMP.

\section{Psychological Changes}

When an athlete is preparing for a major COMP, not only can physiological and performance changes take place, but the athlete may also experience psychological changes due to traininginduced fatigue, outside stressors, and recovery from training. Biddle (1983) identified two psychological factors that highly influence performance in elite level weightlifters: 1) the uncertainty of COMP outcomes and 2) one's perception of COMP importance. Biddle suggests that anxiety rises to a peak at the time of the first lift (i.e., first snatch attempt) and quickly subsides afterward regardless of a successful or missed lift. This idea stems from the weightlifter being concerned if their training and preparation were "enough" for the competition despite the COMP importance. Other conclusions can be drawn from Biddle's study where sudden changes in motivation, sensitivity, or prowess could severely impact the athlete's performance in a negative or positive manner.

Mahoney (1989) investigated elite weightlifters (i.e., officially ranked as $1^{\text {st }}, 2^{\text {nd }}$, or $3^{\text {rd }}$ in the country in any of the 10 weight classes) and non-elite weightlifters (i.e., officially ranked as $11^{\text {th }}$ place or lower, or being unranked) preparing for important COMP. Mahoney found that elite weightlifters scored significantly lower than non-elite weightlifters on negative psychosomatic components associated with intrapersonal sensitivity, depression, psychoticism, global sensitivity, and other sub-scale symptoms. Elite weightlifters have a tendency to show positive 
psychosomatic components being more motivated than non-elite weightlifters, especially female weightlifters, and showing moderate to high levels of self-esteem, vigor, and confidence (Mahoney, 1989). Mahoney also pointed out that elite weightlifters had high variance on specific scales pertaining to anxiety management, concentration, confidence, intrapersonal sensitivity, anger, and stiffness (i.e., feeling less recovered). Specifically, in Mahoney's subsample of elite female weightlifters, anxiety and motivation showed to be positive covariates to performance levels with lower variability compared to male weightlifters. The findings by Mahoney are congruent with the mental health model (Morgan, Brown, Raglin, O’Connor, \& Ellickson, 1987) suggesting that elite weightlifters tend to score more psychologically healthier during normal training periods as opposed to less successful weightlifters within the sport. The mental health model designed by Morgan and associates (1987) along with the findings by Mahoney (1989) indicate that there is a strong correlation to performance based on negative and positive psychopathology, especially when approaching a COMP and during a COMP.

Monitoring an athlete's psychological changes over time can allow the sport scientist and coach to ensure that the athlete is not only physically but psychologically prepared for any COMP. The ability to produce appropriate emotional feelings before a major COMP has been recognized as an important factor contributing to positive or negative performances. Many studies suggest that questionnaires (i.e., Profiles of Mood States [POMS], Recovery-Stress Questionnaire [REST-Q], Daily Analysis of Life Demands for Athletes [DALDA]) can expose subjective alterations in levels of anxiety, feelings of fatigue, stress levels and mood state. Using a questionnaire as a monitoring tool could allow appropriate VL adjustments to be made leading 
up to a COMP (Cockerill, Nevill, \& Lyons, 1991; Mujika et al., 2002; Mujika, 2009; Mujika \& Padilla, 2000, 2003; Mujika, Padilla, Pyne, \& Busso, 2004).

A recent study observing 11 international level weightlifters by Durguerian et al. (2016) observed significant reductions in global recovery scores with higher scores for general wellbeing and emotional stress using the REST-Q-52 questionnaire. This took place during two simulated COMP over a 6 -day period. One group maintained body mass $(n=5)$ whereas the other group was instructed to cut body mass $(n=6)$ between the two COMP. Fatigue scores associated with overall feelings of recovery and vigor was significantly higher in the body mass maintenance group compared to the body mass reduction group. Countermovement jump (CMJ) was used as a secondary performance measurement to psychological changes and actual weightlifting performance. The weightlifters who maintained body mass increased jump height $(\mathrm{JH})$ by $3.9 \%$ while the reduction group reduced body mass by $3.3 \pm 0.6 \mathrm{~kg}$ and saw a decrease in $\mathrm{JH}$ of $3.2 \%$. Although the body mass reduction group showed lower JH and improved psychological inventories, absolute weightlifting performance improved by $1.4 \%$ and scaled COMP performance improved by 3.5\%. However, it is important to note that both groups improved psychological scores overall, while the body mass reduction group had stronger feelings of being fatigued. Large body mass reductions $(>3 \mathrm{~kg})$ attempted close to COMP $(<1$ week) have been shown to be detrimental to performance (Bazyler et al., 2018) and the aforementioned findings suggest that rapid body mass reductions close to COMP can cause the athlete to feel fatigued which will, in turn, impact performance. Thus, while psychological measures provide an indication of COMP preparedness, careful attention also needs to be given 
to dietary strategies along with subjective fatigue measures by weightlifters preparing for COMP.

Sport scientist and coaches should strive to be more aware of their athlete's mood, recovery, and stress state before training and more importantly before or at a COMP. Traveling, different sleeping environments, cutting weight, elevated stress levels, and precompetition anxiety can potentially alter an athletes performance ability (Blumert et al., 2007). Blumert et al. (2007) showed that mood disturbances are highly associated with physiological changes in weightlifters when observing biomarkers such as testosterone (T), cortisol (C), and testosterone-to-cortisol $(\mathrm{T}: \mathrm{C})$ ratio. These biomarkers are typically associated with not only psychological states (i.e., aggression and $\mathrm{T}$, stress and $\mathrm{C}$ ), but induced changes from VL that can influence performance outcomes to a great extent (Siart, Nimmerichter, Vidotto, \& Wallner, 2017). If a negative mood disturbance is reported, focusing on shifting the mood disturbance in a positive manner by relaxing the athlete, using imagery of post-competition success, and reassuring the athlete of their ability would be beneficial.

Outside stressors causing an athlete to feel pressured to compete could potentially pose a serious threat to express performance at an important COMP (Siart et al., 2017). For instance, in a major COMP among a cohort of ballroom dancers, when the dancers were under highly focused conditions that threatened overall placing, significant increases in the physiological stress hormone $\mathrm{C}$ was associated with performance decrements (Rohleder, Beulen, Chen, Wolf, \& Kirschbaum, 2007). However, a recent study observed increased C associated with negative psychological responses and increased $\mathrm{T}$ associated with anger and self-esteem 24 hours and 
immediately before COMP resulting in decreased performance at a national track and field championship (Siart et al., 2017). In weightlifting, a recent study suggests that rises in serum $\mathrm{T}$ before a COMP are influenced by $\mathrm{C}$ activity and could explain a COMP outcome which is dependent on the task, (i.e., first attempt), situational conflicts (i.e., overall placing, attmept selection), and environmental needs (i.e., warm-up area, start number) of the athlete (Crewther, Obmiński, \& Cook, 2018). Thus, weightlifting is a sport that requires the athlete to be focused, have mental acuity and be physically prepared to compete. A weightlifter's motivation and desire to compete could override any physiological phenomenon taking place that would typically be indicative of a positive performance outcome and should be monitored accordingly leading up to and at a major COMP.

\section{$\underline{\text { Physiological Alternations }}$}

A vast amount of literature has purported endocrine system alterations through biochemical and hormonal adaptations induced by short-term and long-term training (Mujika et al., 2004; Viru \& Viru, 2001). Mujika and Padilla (2000) suggest that monitoring biochemical changes and hormonal adaptations indicate if training is being carried out appropriately along with a proper tapering protocol where performance changes can be recognized before a major COMP (Coutts \& Murphy, 2007; Maynar et al., 2010). In weightlifters and other athletes alike, indications of physiological stress, recoverability, and adaptability have been associated with potential blood borne biomarkers such as T, C, T:C ratio, sex-hormone-binding-globulin (SHBG), T:SHBG ratio, interleukins, creatine kinase (CK), high-sensitivity C-reactive protein (hsCRP), decorin, myostatin, and insulin-like growth factor-1 (Bazyler et al., 2018; Maynar et al., 2010; Mujika, 2009; Mujika et al., 2004; Storey \& Smith, 2012; Viru \& Viru, 2001). 


\section{$\underline{\text { Biochemical Fluctuations }}$}

Throughout the literature, endocrine fluctuations along with the response to changes and adaptation to training in weightlifters are limited to anabolic and catabolic hormones along with cytokines and immune responses. However, it should be noted that most of the literature dealing with endocrine responses and adaptations for weightlifters majorly pertains to male weightlifters (Storey \& Smith, 2012). In this section of the review, the authors highlight the most relevant endocrine alterations relative to male and female weightlifters that are most indicative of negative and positive performance changes.

\section{Endogenous Steroid Hormones}

Hormones such as T, C, T:C ratio, and SHBG have received a lot of attention throughout the literature dealing with weightlifters which have shown strong relations with performance fluctuations and observed trends (Storey \& Smith, 2012; Viru \& Viru, 2001). The T:C ratio has been suggested to be the strongest indication of physiological stress imposed by the training programs and shows an inverse relationship to volume-load in both male and female weightlifters (Haff et al., 2008; Storey \& Smith, 2012). This relationship can be used to provide indications of an athlete's anabolic-catabolic state before and during the taper in order to produce corresponding positive performance outcomes (Storey \& Smith, 2012; Tønnessen et al., 2014). However, although biochemical measurements provide valuable information on physiological responses to training, sometimes physiological phenomena in elite athletes do not adhere to the evidence provided within the literature (Murach \& Bagley, 2015; Wu et al., 2008). 
In general, approximately $2 \%$ of plasma testosterone exist in a free state form with the remaining $98 \%$ being bound to albumin and SHBG. In turn, increases in SHBG reduces the excretion rate of androgenic-anabolic activity in an attempt to preserve $\mathrm{T}$ availability for muscle tissue (Hooper et al., 2017). Because increases in T are considered important during recovery periods (i.e., tapering, active recovery), SHBG will increase as a compensatory response to the increased demand for $\mathrm{T}$ at the muscular level. For example, T, C, and SHBG were observed in 19 national team weightlifters over a 20-week period (Maynar et al., 2010). Contrary to the literature, $\mathrm{T}$ and $\mathrm{C}$ were unaltered, but $\mathrm{SHBG}$ showed a significant rise that was explained due to an increase in protein synthesis and served as a compensatory mechanism to facilitate the constant androgenic-anabolic activity. However, the methods and statistical analysis of this study may have caused the serum levels to disagree with previous literature. Another study showed no significant changes in T:C or SHBG in elite male weightlifters after either a 1 or 4-week taper, yet performance still improved by 8.0 and $17.5 \mathrm{kgs}$ during COMP (Mujika, 2009; Stone et al., 1996). Even in other strength-trained athletes, Izquierdo et al. (2007) observed no significant changes in total-testosterone (TT), free-testosterone (FT), and C concentrations after 16 weeks of training that included a 4-week taper. Therefore, based on these equivocal findings, biochemical markers are extremely diverse and unpredictable but monitoring changes within each athlete may help explain potential performance outcomes for both male and female weightlifters.

Busso and Häkkinen et al. $(1990,1992)$ observed elite weightlifters over a 2-year period using mathematical modeling that was developed to predict and explain performance changes over time with experimental data and actual serum markers. Statistical changes were observed in $\mathrm{T}: \mathrm{C}$ ratio and $\mathrm{SHBG}$ at specific points throughout the first year and percent change correlated 
well with actual performance for each individual lifter. In the second year, changes in the same serum markers were not as clearly defined as the previous year using the same protocol. This information provides insight into the adaptability in well-trained high-level athletes and suggests that athletes competing at a high-level can acquire higher training thresholds to improve performance later on over a year of training. If the same stimulus is applied with the aim of receiving a performance gain, then the stimulus will be insufficient resulting in maintained or decrements in performance. In fact, recent evidence suggests a sustained, acute hormonal response ( $\mathrm{T}, \mathrm{C}$, Growth Hormone) is needed to promote further adaptations in hypertrophy and maximal strength (Hooper et al., 2017; Walker, Häkkinen, Haff, Blazevich, \& Newton, 2017).

Fry et al. (1994) completed a study similar to Busso and Häkkinen et al. $(1990,1992)$ and suggest that the exercise-induced changes in T concentrations during a 1-week overreach were augmented after 1-year of continuous training. The athletes did not respond to the same degree after one year of training with the same stimulus prescribed again. Thus, the authors suggest that well-trained athletes need more physiological disturbance and higher rigorous training stress (i.e., overreach microcycles) in order to receive a greater rebound effect (i.e., supercompensation). Without the appropriate heightened training stimulus, biochemical changes cannot take place in order for the athlete to rebound later on. Determining and distinguishing $\mathrm{T}$, $\mathrm{C}, \mathrm{T}: \mathrm{C}$ ratio, and SHBG concentrations during normal training periods along with psychological state, muscle tissue adaptation, strength levels and explosiveness should be quantified in order to potentially forecast performance outcomes. It is likely that the aforementioned studies showed inconsistent changes in T, C, T:C ratio, and SHBG due to the adaptability of elite level weightlifters. Therefore, these steroid hormones from an individual perspective may provide the 
sport scientist and coach with information that explains performance alterations even if substantial changes in serum hormone levels do not occur. With the unique genetics, physiological differences, and training practices in high-level weightlifters, determining a biomarker to express individual recovery, fatigue, and preparedness that is indicative of performance alterations would be invaluable for monitoring an athlete's long-term development.

\section{$\underline{\text { Muscle Damage Biomarkers }}$}

Resistance exercise is known to cause myofibril disruptions, especially during eccentric muscle actions resulting in inflammatory responses modulated by cytokines (Izquierdo et al., 2009). Gentles et al. (2017) state that weightlifters rarely abstain from training or exercise for several days and doing such may allow markers of muscular damage and stress such as hsCRP and $\mathrm{CK}$ to remain elevated for a period of time post-training. Therefore, if either of these markers is elevated post-training then the return to baseline will occur exceedingly fast (Viru \& Viru, 2001). Perhaps, due to the type of training and adaptability high-level athletes possess, this may explain why the inflammatory response is less pronounced in high-level weightlifters when compared to other athletic populations and inactive groups throughout the literature.

Stewart et al. (2007), along with several other researchers, have shown trends in physical activity levels and hsCRP. The authors indicated that other large cross-sectional studies have used recreationally trained groups along with older and younger inactive groups and found, regardless of age, indications that inactive individuals have significantly higher levels of hsCRP. Stewart et al. (2007) also pointed out that low body fat percentage is strongly associated with serum hsCRP concentrations. In regards to weightlifting, high-level male and female athletes are 
typically leaner and very active (Stoessel et al., 1991; Stone \& Kirksey, 2000); therefore, it is expected that hsCRP would be low in this athletic population. Given these findings, it is possible that changes in weightlifter's body fat could partly explain training-induced changes of hsCRP considering the markers sensitivity to fat accumulation associated with chronic inflammation.

Bloodborne markers such as hsCRP and $\mathrm{CK}$ have been shown to reflect acute and chronic levels of stress and fatigue (Hecksteden et al., 2016). Elevated acute phase proteins and cytokines such as CK from training-induced stress can be measured dependably, especially hsCRP immediately after an intense training cycle. High constant hsCRP levels and cytokines are not typically found in the literature for athletic populations; however, if an athlete starts experiencing chronically high levels of hsCRP and/or CK then training adjustments should be made immediately to prevent overtraining (Halson et al., 2002; Meeusen et al., 2013). Gentles et al. (2017) also observed that percent change of CK in weightlifters was related to weekly training stress, but the actual CK concentration was not representative of the most recent measurement of VL or training intensity. Although CK did not appear to respond to VL acutely, it showed a very strong correlation $(\mathrm{r}=0.86)$ with the amount of work completed over a four-week training block. It was also noted that hsCRP concentrations appeared to rise and fall more synchronously with VL bi-weekly and may have been influenced by pronounced elevations observed in CK concentrations. For strength-power athletes using strength training exercises, together hsCRP and CK are fatigue-dependent markers that can potentially alter an athlete's recoverability (Hecksteden et al., 2016). Hecksteden et al. (2016) note that these fatigue-dependent markers can plausibly confirm the amount of training strain an athlete has undergone from the previous training blocks. 
Based on the previous sections, it is evident that observing psychological state and steroid hormones could possibly reveal important information acutely and chronically in individual weightlifters. If an athlete's performance levels are increasing but self-reported training stress is high perhaps inflammatory markers or cytokines are responsible for the athlete's current psychological state. Understanding which markers are disrupted most frequently within each athlete may aid in reducing stress and improving recovery, especially for weightlifters preparing for a major COMP. Therefore, special considerations should be taken into account for not only individual weightlifters, but specifically for female weightlifters due to menstrual cycle variability and resulting hormonal and performance changes.

Considerations for the Female Athlete

During certain phases of the menstrual cycle, female athletes can be extremely sensitive to training stressors physically, hormonally, and psychologically (Hamilton, 2012; Sarwar, Niclos, \& Rutherford, 1996). Hamilton (2012) notes that some female athletes do not experience significant changes in performance whereas others have extreme difficulty performing, recovering, and mitigating fatigue. The athlete's dysmenorrhea could be severe enough to disrupt training and performance. Therefore, it is important to monitor and track hormonal changes and psychological states of female athletes relative to their cycle when preparing for a COMP.

The menstrual cycle lasts anywhere from 31 to 25 days and on average of 28 days for most female athletes (Hamilton, 2012). The main component that affects the female athlete during menstruation is changing in hormone activity that influences other psychological and physiological phenomena (Kenney, Wilmore, \& Costill, 2015). The menstrual cycle is divided 
into two main phases of the follicular phase and the luteal phase. The follicular phase is comprised of the flow phase followed by the proliferative phase. Subsequently, the luteal or secretory phase takes place which begins with the ovulation period. During the first phase, high levels of follicle-stimulating hormone (FSH) are typically noticed. During the second phase peak concentrations of luteinizing hormone ( $\mathrm{LH})$ is noticed followed by a second rise in FSH (Hamilton, 2012). The balance of FSH and LH dictate the regularity of the menstrual cycle (Cook, Crewther, \& Smith, 2012; Sarwar et al., 1996). The release of FSH and LH have a secondary effect on other hormones such as estrogens, endorphins, dopamine, catecholamines, C, and T concentrations (Cook et al., 2012; Llewellyn, 2010). The differences amongst men and women in athletics are obvious when observing serum concentrations of hormones such as $\mathrm{T}$ (Llewellyn, 2010). When considering $\mathrm{T}$ during the menstrual cycle, peak concentrations coincide with other sex hormones as the female goes into ovulation (Hamilton, 2012). Therefore, monitoring fluctuations of $\mathrm{T}$ according to the female athlete's menstrual cycle could allow COMP preparation to be carried out appropriately (Stone, Stone, \& Sands, 2007).

Strength and power training for female athletes should be an area of important consideration for the sport scientist and coach and should consider different phases of the menstrual cycle influence the athlete's ability to a great extent (Holloway \& Baechle, 1990; Stone, Plisk, \& Collins, 2002). During ovulation, serum concentrations of testosterone have been shown to be significantly elevated relative to normal levels (Phillips, Sanderson, Birch, Bruce, \& Woledge, 1996; Sarwar et al., 1996). A recent study showed serum T, C, and T:C was considerably higher in elite female athletes compared to non-elite female athletes during ovulation (Cardinale \& Stone, 2006; Cook et al., 2012). Strength levels during ovulation are high coinciding with higher 
force generation capabilities and power outputs compared to normal weeks of training (Kishali, Imamoglu, Katkat, Atan, \& Akyol, 2006). Another study showed that during ovulation, female athletes showed an $11 \%$ increase in quadriceps and handgrip strength, which are highly-relevant in weightlifting (Sarwar et al., 1996). However, although performance may be high, so is the risk of injury due to increased estrogen levels.

Performance differences between men and women in sports are substantial, partly due to the different physiological and endocrinological characteristics of both sexes. In a study by Bosco (1997), it was reported that altered performance responses induced by a maximum strength training session were completely different between the men and women. However, Bosco's study lacked indication of what portion of the menstrual cycle the female athletes were in. A study by Haff et al. (2008) observed biochemical and performance changes in female weightlifters coinciding with other reports that resting $\mathrm{T}$ concentrations did not significantly change based on the menstrual cycle. However, it has been also noted that serum $\mathrm{T}$ concentrations have a very large inter-individual difference in high-level women athletes (Cook et al., 2012; Haff et al., 2008).

A more recent study by Caporale (2017) observed four Fiamme Azzurre national team weightlifters over a 10-month period which ended with a major COMP (i.e., Italian Championships 2013 in Modena). The female athletes were observed on their psychological, biochemical, and physical performance during training and COMP. Throughout the study, several important variables were noted which influenced the performance of each lifter such as body temperature, subjective breathing ability, hematological parameters such as iron levels, 
ability to produce power (i.e., nervous system as a modulator of responses to workloads and recovery index) central regulation of perceived effort, and psychological aspects. Cyclic performance decrements were noticed during the most fertile 5-day period prior to ovulating and performance improvements were noticed 1-2 days before and during the ovulation window.

Psychological inventories and biochemical evaluations showed high variations relative to each female's cycle. Once inter-individual variability is determined, tapering strategies designed to peak during a female's ovulation window would be ideal. Due to the reductions in VL and elevated T concentrations, this could generate a higher capacity to express performance which is commensurate with the demands for elite sports such as weightlifting. Evaluations and performance results of female weightlifters during the menstrual cycle seems to be contrasting throughout the literature much like the previous discussion of the $\mathrm{T}: \mathrm{C}$ ratio in male weightlifters. Regardless, training and performance differences still remain a subject of discussion even though variability is high amongst men and women. It is advised to follow an athlete's changes more than once to determine if variations are due to the menstrual cycle or other confounding factors. These differences are clear in performance outcomes but are a product of differences in muscular, hormonal, and psychological characteristics pertaining to absolute muscular size, power, strength, and speed. Determining each lifters inter-individual changes and trends would be advised in order to allow each athlete to achieve their highest performance potential regardless of sex. 


\section{$\underline{\text { Muscular Alterations }}$}

Some of the most important changes that take place in the weightlifting athlete are muscular and neuromuscular adaptations that can have positive or negative effects on performance. Observing muscle fiber type in weightlifters would be beneficial and contribute to the trivial body of literature that currently exists. Conversely, such observations are typically considered to be too invasive for the athlete which can interfere with the training process. Therefore, observing whole muscle size alterations and neuromuscular function is vital for monitoring weightlifting, especially those competing at the international and national level.

\section{Muscle Architecture}

The vastus lateralis is generally considered to be the largest and most powerful section of the quadriceps musculature particularly in athletes (Secomb, Nimphius, Farley, Lundgren, Tran, Sheppard, 2015). Monitoring muscle architecture changes in weightlifters can be used to infer changes in muscle function, force production capability, contraction velocity, and hypertrophic responses from training. Magnetic resonance imaging is considered to be the "gold "standard for observing muscle architecture in humans. However, in order to avoid disrupting the training process, a fast non-invasive cost-efficient, reliable, and valid alternative is using ultrasonography (Scanlon et al., 2014).

Male and female weightlifters, bodybuilders, and powerlifters display an extraordinary amount of muscular development compared to non-athletic persons (Katch, Katch, Moffatt, \& Gittleson, 1980). Thus, observing muscle architecture through ultrasonography can be carried out with male and female athletes being physiological and performance characteristics have been 
shown to be relatively similar between sexes (Lewis, Kamon, \& Hodgson, 1986). Although possessing greater muscle mass and size can differentiate weightlifters from normal control subjects, it does not appear to differentiate well between elite and sub-elite weightlifters (Funato et al. 2008; Di Naso et al. 2012). However, vastus lateralis muscle cross-sectional area (CSA) and muscle thickness (MT) have been shown to be strong indicators of movements often used by weightlifters such as vertical jumps, isometric-mid thigh pull (IMTP), back squat, deadlift, and power clean (Bazyler et al., 2018; Brechue \& Abe, 2002; McMahon, Turner, \& Comfort, 2015; Secomb et al., 2015; Zaras et al., 2016).

It is common in high-level male and female weightlifters to be relatively short in stature and limb length while possessing relatively lean and muscular physiques corresponding with predominant mesomorph-endomorph somatotype (Stoessel et al., 1991; Stone \& Kirksey, 2000). The vastus lateralis muscle is typically observed in strength-power athletes that strongly considerers $53 \%$ to $65 \%$ of the tissue to be predominately fast-twitch fibers (Clarkson, Kroll, \& McBride, 1980). Specifically, in American national class weightlifters, the cross-sectional area of type II muscle fibers is considerably larger when compared to other strength-power athletes such as powerlifters and throwers (Fry et al., 2003; Gollnick, Armstrong, Saubert, Piehl, \& Saltin, 1972). This difference is advantageous for producing high levels of force quickly because type II fibers possess a greater capacity to generate power per unit of muscle CSA when compared to type I fibers (Andrew C. Fry et al., 2003; Gollnick et al., 1972). Because the vastus lateralis in weightlifters has been shown to be predominately type II muscle fibers, this gives insight into the relationship between performance and changes (i.e., increase in muscle CSA and MT) in lower extremity musculature (Fry et al., 2003). It has been suggested that as muscle CSA 
and MT increases maximum strength increase linearly with these changes which are vital characteristics to have for strength-power athlete's COMP demands (Scanlon, Fragala, Stout, Emerson, Beyer, Oliveira, Hoffman, 2014).

In addition, weightlifting performance (i.e., snatch and C\&J) has been strongly correlated to type IIA percent content ( $\mathrm{r}=0.94)$ along with type IIA muscle CSA ( $\mathrm{r}=0.83)$ (Fry et al., 2003). Following a taper prior to a major COMP, a restoration of type IIX fiber content has been shown to occur in other athletes (i.e., swimmers, runners, and cyclists) which is attributed to the reduction in VL while maintaining relatively high intensities (Fry, 2004; Staron et al., 1990). However, observing this tapering-induced shift from type IIA to type IIX within fiber content has not been explored in weightlifters. Monitoring fiber type shifts would be extremely beneficial, especially during a taper, but in order to carry this procedure out repeatedly over a short period of time would likely interfere with the athlete's training and peaking strategies (Storey \& Smith, 2012). Therefore, observing structural components of the tissue via MRI or ultrasonography with an understanding of changes taking place that are dependent on molecular phenomenon will provide the most reliable and valid observation to discern changes that are taking place during the taper. Coupling observed changes in muscle architecture with neuromuscular alterations through performance testing would provide insight into the athlete's preparedness level prior to a COMP.

Maximal voluntary isometric peak force (IPF) is strongly related to weightlifting performance and other dynamic muscle actions (Stone et al., 2005). Isometric peak force (IPF), isometric rate of force development (IRFD) between 200-250ms, and contractile impulse (CI) 
are influenced by a number of morphological factors including muscle fiber type, tendon properties, pennation angle, and muscle thickness (Haff et al., 2005; Kauhanen, Garhammer, \& Häkkinen, 2000; Stone et al., 2005). A large anatomical pennation angle allows more sarcomeres to be arranged in parallel (Stebbings, 2015), thus increasing muscle CSA and MT which are both strongly associated with an athlete's ability to express maximum strength and power (McMahon, Turner, \& Comfort, 2015). Theoretically, an increase in pennation angle results in a large muscle CSA thereby further improving contractile IRFD and CI (Storey, Wong, Smith, \& Marshall, 2012). Storey et al. (2012) suggest that maintaining muscle size does not necessarily change the athlete's ability to express maximum strength. A recent study observing a high-level female weightlifter found that large reductions in body mass $(>3 \mathrm{~kg})$ resulted in decreased muscle CSA which may explain underperformance at a national championship (Bazyler et al., 2018). Therefore, during a taper leading to a major COMP, it is important to monitor changes in strength levels, muscle architecture, and surrogate performance simultaneously to ensure that the athlete's performance ability is not diminishing below baseline levels.

Scanlon et al. (2014) observed increases in strength corresponding to increased vastus lateralis muscle CSA, but CSA increases of vastus lateralis did not correspond to changes in MT. This study was only carried out for 6 weeks and the authors noted that other observations have shown MT increases of $14.8 \%$ in men and women over 12 weeks suggesting that longer studies of $\geq 12$ weeks are needed to determine this relationship. Other conclusions from this study indicate that MT seems to change more acutely ( $\leq 6$ weeks) as compared to muscle CSA being a more chronic muscular measurement. The authors also suggest that muscle CSA provides a more sensitive assessment of total muscle hypertrophy as it has been reported to be better correlated 
with hypertrophic and force producing characteristics (i.e., RFD) associated with muscle size and production ability. A noticeable increase or decrease in MT alone may be indicative of the athlete's level of training stress and could correspond with a concurrent inflammatory response. In a recent study by Raeder et al. (2017) the authors observed suppressed countermovement jumps $(\mathrm{CMJ})$ during training and an active recovery period in German national weightlifters. This study indicated the importance of not only lower extremity muscular fatigue, but also how easily neuromuscular fatigue is accumulated throughout training. Nevertheless, while the ability to express maximum strength and power is closely associated with overall muscle mass, muscle CSA, and MT, there is still a significant neural component that should not be overlooked (Brechue \& Abe, 2002).

\section{$\underline{\text { Neural Adaptations }}$}

Increases in muscle CSA following strength training is a general adaptation coinciding with neural adaptation in high-level strength and power athletes (Castro, McCann, Shaffrath, \& Adams, 1995; Häkkinen, Pakarinen, Alen, Kauhanen, \& Komi, 1988). If further neural adaptations do not take place the ability to increase force production will likely not match changes, if any, in muscle CSA measurements and will remain the same (Brechue \& Abe, 2002; Häkkinen et al., 1988; Sale, 1988). As previously mentioned, COMP performance and ability are strongly correlated to type IIA muscle fiber content and muscle CSA in weightlifters (Fry et al., 2003). Strong relationships between maximal strength (i.e., IPF), peak power (PP), and jump height $(\mathrm{JH})$ have concurrent strong relationships to weightlifting performance (Carlock, Smith, Hartman, Morris, Ciroslan, Pierce, Newton, Harman, Sands, Stone, 2004; Haff et al., 2005; Stone, Sands, Pierce, Carlock, Cardinale, Newton, 2005). In order to express these performance 
characteristics to the greatest capacity fatigue must be unmasked through tapering in order to see performance changes. After a 3-week taper, Trappe et al. (2001) observed 30\% higher IPF, 67\% faster shortening velocity, and $250 \%$ higher power output in single Type IIA muscle fibers in highly trained collegiate swimmers. On average, Type II fibers produce twice as much peak power as Type I fibers before tapering and five times as much peak power after the taper, indicating that neuromuscular adaptations at the single-fiber level are paramount for performance (Mujika, 2009; Trappe et al., 2001). However, these findings have not been well studied in strength-power athletes. A review by Malisoux, Francaux, and Theisen (2007) concluded that tapering has relatively little influence on the size and force characteristics of Type I muscle fibers, whereas Type IIA and IIX fibers seem to be more responsive to performance improvements after the taper. Assuming these single fiber alterations are expressed at the whole muscle level, increased muscle CSA during the taper could manifest itself in greater force production and power output after fatigue dissipates.

Andersen and Aagaard, (2000) demonstrate strength training induces a myosin isoform shift from type IIX to IIA, whereas the reduction in training VL can cause an overshoot phenomenon from type IIA back to type IIX in sedentary males. However, this overshoot was observed over a 3-month detraining period and performance measures (i.e., isometric strength) returned to baseline despite the fiber type shift. Being there has been strong evidence indicating the predominance of type IIA fiber content in weightlifters, this theoretical overshoot phenomenon and a shift in myosin isoforms could be vital to express peak performance (Fry et al., 2003). However, alterations in Type II fiber morphology, enzymatic activity, and contractile properties 
help explain increases in performance after the taper beyond the overshoot phenomenon (Andersen \& Aagaard, 2000; Murach et al., 2014; Neary et al., 2003; Trappe et al., 2001).

In a recent review by Maffiuletti et al., (2016) the authors indicate that the surrogate measure of neuromuscular function is RFD. The authors suggest that RFD is strongly related to sportspecific performance based task and is extremely sensitive to detecting acute and chronic neuromuscular function. Performing maximal isometric strength task is not only less taxing on the athlete compared to maximal dynamic task (i.e., 1RM back squat) but also indicative of weightlifting performance (Beckham et al., 2013; Haff et al., 2005). During the IMTP, IRFD and IPF have been shown to be beneficial metrics for monitoring neuromuscular function and changes in strength levels for male and female weightlifters (Hornsby et al., 2017). In turn, studies using trained weightlifters have revealed relationships exist between muscle CSA and maximum strength (i.e., IPF) (Ikai \& Fukunaga, 1968; Kawakami, Abe, \& Fukunaga, 1993). Another study observed the vastus lateralis MT of high-level powerlifters and found very strong relationships between squat performance $(\mathrm{r}=0.91)$ and deadlift performance $(\mathrm{r}=0.90)$, which are two movements often used in normal training regimes for weightlifters. If neuromuscular fatigue is present, IRFD and IPF will likely be suppressed resulting in decreased motor output. If a weightlifter's motor output is compromised from accumulated fatigue, technique will begin to change early with often marked increases in horizontal bar displacement (e.g., bar drifting away from the body) (Stone, 1993). Increases in horizontal bar displacement for weightlifters can be explained by residual fatigue from an overreaching phase indicating that the athlete is not recovering and adapting to the stimulus and a taper is needed. Observations in well trained Finnish powerlifters showed greater muscle activity in the quadriceps for higher-level 
powerlifters compared to the less successful powerlifters after a 1-week taper (Häkkinen, Alén, \& Komi, 1984). Although weightlifters and powerlifters are comparable, discrepancies between the athletes lie between the rate at which each athlete generates maximum force in order to be successful in COMP. In preparation for a major COMP, if a weightlifter is experiencing suppressed RFD and increases horizontal bar displacement, then beginning the taper is encouraged prior to COMP.

Male and female weightlifters have both shown significant improvements in both PF and RFD abilities that can be attributed to training programs designed to meet COMP demands (González-Badillo et al., 2006; Haff et al., 2005, 2008; Stone et al., 2005). These findings suggest that frequent high-intensity training used by weightlifters effectively increase muscular strength and power concurrently. Generally, IPF and RFD in male weightlifters are $15-20 \%$ and 13-16\% greater when compared to other strength-power athletes (Funato, Kanehisa, \& Fukunaga, 2000; J. Garhammer, 1980; Garhammer \& Gregor, 1992; Storey \& Smith, 2012). This improved muscular function may arise due to an enhanced voluntary and/or reflex-induced neural activation of motor units and a selective recruitment of fast twitch motor units (Storey \& Smith, 2012) which may be attributed to the maintenance of muscle CSA and MT once the athlete is properly recovered.

Throughout training for weightlifters, the COMP lifts and derivatives have demonstrated some of the highest absolute and relative PP reported in the literature (Garhammer, 1980). Further, performance tests such as vertical jump tests have purported that PP and JH are significantly higher in weightlifters when compared to other athletes (Funato, Kanehisa, \& 
Fukunaga, 2000; Garhammer, 1980). This performance adaptation can likely be explained by training specificity. However, if a weightlifter begins to produce lower PF, high fluctuating RFD, and suppressed PP along with maintained muscle CSA and MT during monitoring and performance testing, then the athlete's training program should be monitored accordingly to look for signs of training beyond an overreached state. These findings demonstrate and highlight the importance of monitoring morphological, neuromuscular and performance changes during a taper in high-level weightlifters preparing for a major COMP.

\section{$\underline{\text { Performance Adaptation }}$}

Maximal strength and power testing has been used extensively throughout the literature to indicate an athlete's preparedness (Beckham et al., 2013; Carlock et al., 2004; Haff et al., 1997, 2005, 2008; Häkkinen et al., 1986, 1987; Mcbride, Triplett-Mcbride, Davie, \& Newton, 1999; Nuzzo, McBride, Cormie, \& McCaulley, 2008; Stone \& Kirksey, 2000; Vizcaya, Viana, del

Olmo, \& Acero, 2009). The body of literature shows that weightlifters are superior athletes when it comes to performance testing such as squat jump (SJ), CMJ, and IMTP testing. These superior results may be due to genetics, but it is also likely due to specific training programs and COMP demands (Stone \& Kirksey, 2000). Vertical jump testing can be used to detect fluctuations in power output, JH and dynamic RFD capabilities throughout training cycles and leading up to COMP. Vertical jumps are the preferred monitoring tool for assessing weightlifting COMP preparedness due to their limited interference with training.

Vertical jump tests are useful for predicting weightlifting ability due to biomechanical similarities between the activities (J. Garhammer, 1980, 1993; John Garhammer \& Gregor, 
1992). Jump height, specifically SJ height (SJH), has been shown to be strongly related to absolute and scaled weightlifting performance and can be used as a secondary performance measure to monitor weightlifting performance in male and female weightlifters (Travis, Goodin, Suarez, \& Bazyler, 2017).

\section{$\underline{\text { Secondary Measure of Performance }}$}

It is impractical for weightlifters to regularly perform maximal attempts on COMP lifts, and would potentially result in overtraining or injury. Therefore, a secondary measure to monitor weightlifting performance is needed. Laboratory testing including SJ, CMJ, deep squat jump, and IMTP have all been shown to have positive relationships with snatch, $\mathrm{C} \& \mathrm{~J}$, absolute total, and Sinclair Total for male and female weightlifters (Bazyler et al., 2018; Beckham et al., 2013; Carlock et al., 2004; Haff et al., 2005; Stone et al., 2005; Travis et al., 2017; Vizcaya et al., 2009). Physiological measures of serum blood markers, vastus lateralis muscle CSA, and MT has also been shown be indicative of monitoring test and COMP performance (Brechue \& Abe, 2002; Gentles et al., 2017; Haff et al., 2008; Loenneke, Wilson, \& Wilson, 2010; Secomb et al., 2015). Although performance and physiological tests can be indicative of performance, using specific laboratory tests often such as SJ, CMJ, and IMTP seem to be better predictors of weightlifting performance.

Metrics from IMTP such as IPF and IRFD are strong indicators of maximal strength and neuromuscular fatigue in male and female weightlifters, respectively (Beckham et al., 2013; Haff et al., 1997; Stone et al., 2005). Acute and chronic neuromuscular fatigue along with power production capabilities can also be monitored with unloaded and loaded SJ and CMJ testing in 
weightlifters (Hornsby et al., 2017). Findings from Carlock et al. (2004) and Travis et al. (2017) both suggest that unloaded SJH and peak power (PP) have direct correlations with weightlifting performance. However, Travis et al. (2017) specifically found that unloaded SJH in male and female weightlifters is a strong correlate to scaled weightlifting performance comparable to CMJ and IMTP. Further, loaded SJH (20kg) has been shown to be sensitive to fatigue from alterations in training VL in high-level weightlifters preparing for a COMP (Bazyler et al., 2018; Hornsby et al., 2017). Therefore, sport scientist and coaches can use the SJ as a monitoring tool to determine peak performance, especially during a taper for weightlifters to ensure fatigue is decaying at the appropriate rate to express performance later on.

\section{Tapering Effects on Competition Performance}

Throughout the literature, the results are confounding when deciphering post-taper performance improvements relative to psychological and physiological changes (Raglin, Koceja, Stager, \& Harms, 1996). Raglin and associates (1996) point out that neurological adaptation indeed plays the most important role in performance improvements resulting from the taper, which is vital for weightlifting performance. This study along with others (Freitas, Nakamura, Miloski, Samulski, \& Bara-Filho, 2014; Trinity et al., 2006) suggest that if no improvements in performance are detected then this result could be due to the pre-taper overreach stimulus. If the overreach is not sufficient enough to induce a proper dose of psychological and physiological stress and disturbance for the athlete, then supercompensation before or at the COMP may not occur. In individual sports at the national and international level, performance changes of $1.5 \pm 0.5 \%$ are considered significant and are needed in order to see a positive performance change (Mujika, 2009). Mujika et al. (2002) followed 99 Olympic swimmers from different 
countries who competed in the Melbourne Grand Prix Series and 21-28 days later in the Sydney Olympics. Ninety-one out of the 99 swimmers improved swimming performance following a 3week taper with an overall performance improvement of $2.18 \%$. This performance change showed that male athletes responded better to the tapering model rather than the females athletes (Mujika et al., 2002).

In strength-power athletes, a 4-week overreach and tapering period increased throwing performance and training characteristics (1RM PC, IMTP PF, dynamic MTP RFD) in track and field male and female throwers (Zaras et al., 2014, 2016). Yet, Mujika and colleagues observed no changes in performance during the Olympic Games compared to an event 3 weeks prior in swimmers (Mujika et al., 2002; Mujika, 2009). It was suggested that the unchanged performance was attributed to a lack of proper planning and strategy during the taper. Therefore, it is crucial for a sport scientist and a coach to understand how an athlete responds to training in order to give the athlete a window of opportunity to peak when necessary.

Psychological and physiological impairments from the training process may result in performance decrements. Therefore, the key objectives for an effective taper that improves performance are to 1) maximally reduce accumulated physiological and psychological stress and fatigue of daily training and 2) to restore training tolerance so that training-induced adaptations can be expressed on COMP day (Brechue \& Abe, 2002; Mujika, 2009). Generally, a performance improvement of 2-3\% should be expected in normal athletes whereas changes from 0-6\% can be observed in high-level well-trained athletes (Mujika \& Padilla, 2003; Pyne et al., 2009). An athlete who is competing at the elite level (i.e., international competitor consistently 
placing within the top 8) acquiring a performance change within this range could realize a difference as much as first to fourth place. For example, Mujika and associates showed that the actual difference between the gold medalist and the fourth place finisher at the international level was $1.6 \%$ while the difference between $3^{\text {rd }}$ and $8^{\text {th }}$ place was $2.0 \%$ (Mujika \& Padilla, 2003; Pyne et al., 2009). Therefore, an effective taper that elicits as much as $1 \%$ gain in high-level athletes can make a substantial difference in the COMP outcome (Mujika, 2009). Specifically, for weightlifters competing at the highest level, the difference between $1^{\text {st }}$ and $4^{\text {th }}$ place at the World Championships in 2017 for all weight classes was 5.2 $\pm 2.2 \%$. However, although this sample is considering the elite of the elite, understanding the variability and fluctuations in each weightlifter's performance ability is vital in order to determine a meaningful positive or negative change.

\section{Smallest Worthwhile Change}

Winning a gold medal in a major international championship requires not only outstanding athletic ability and long-term training progression, but it also involves the athlete achieving peak performance at the right time (Tønnessen et al., 2014). One study that observed variability of elite male and female weightlifters leading up to the 2000 Olympic Games found that the variation for snatch was $2.5 \%$ and 3.75 , for $\mathrm{C} \& \mathrm{~J} 2.5 \%$ and $3.5 \%$, and absolute weightlifting total was $2.5 \%$ and 3.2\%, respectively (McGuigan \& Kane, 2004) under the assumption that these athletes were in a peaked state for COMP. Therefore, determining an individual athlete's smallest worthwhile change (SWC) in a sport such as weightlifting could be the difference between reaching peak performance and being on the podium or completely missing the window of opportunity. McGuigan and Kane (2004) pointed out that SWC performance improvements 
are about half the variation for of the within-athlete variation in swimmers and track and field throwers.

Hopkins (2000) suggest using the SWC as a performance tool to determine a high-level athlete's progress from COMP to COMP and throughout the training process. This information can allow the sport scientist and the coach to see how well the athlete is responding not only to the normal training protocols but also how the athlete is responding to prescribed tapering methods. Findings by Mujika et al. (2002) indicated that mean performance and variability of female athletes were greater during a first COMP than the mean average of males during the second COMP over a training year. This agrees with the slightly higher degree of variation in elite female weightlifters versus male weightlifters observed by McGuigan \& Kane (2004). Thus, possible implications may be made regarding weightlifters and high-level females may peak before high-level males. Finding the SWC of each athlete throughout the training process prior to the peaking phase is important for understanding how the athlete copes with training and recovery.

An idiographic approach to all athletes assumes that no two elite athletes are the same (i.e. male-to-male, female-to-female, or male-to-female) and should always be studied as separate entities. Forecasting performance by using dynamic linear models can assist in predicting performance based on trends found during the normal training phases leading up to a major COMP (Barnett, Cerin, Reaburn, \& Hooper, 2010). This may also allow sport scientist, coaches, and the athlete to find positive and negative subjective and objective trends associated with performance. Using performance measures indicative of actual performance such as IMTP, CMJ, 
and most importantly SJ can be used in monitoring performance changes because high-level weightlifters generally do not compete often over a full training year. In regard to international weightlifting COMP, there is typically only $2.5 \mathrm{~kg}$ separating lifters in the top 5 places, thus the smallest variation in performance can mean the difference between securing a medal or failing to place in the top 5 (McGuigan \& Kane, 2004). The data from McGuigan and Kane (2004) shows that an enhancement in performance has a substantial effect on the medal prospects of a top athlete only if the enhancement is at least half of the coefficient of variation in performance between two successive COMP. The authors also propose that the variation in performances is much greater for athletes who only compete below the national level compared to the international level weightlifters.

Despite sex differences at the elite level (McGuigan \& Kane, 2004), the training model that showed the most meaningful performance changes was training with a peak load during the first week followed by a slow decay afterward in experimental, observational, and mathematical modeled studies (Mujika, 2009; Pritchard et al., 2016). However, it was also suggested that a moderate training load during the overreach can be sustained during the taper which will be beneficial during the earlier part of an athlete's career and larger increases in training load during the overreach with more progressive reductions (i.e. fast decay) may be more beneficial with training age. This consideration is important for athletes competing at the national and intentional level (i.e., high-level). Throughout the tapering literature it is suggested that for strength-power athletes exponential tapers with a fast or slow decay improves performance to the greatest magnitude in high-level strength-power athletes whereas step tapers tend to be better suited for sub-elite and less successful strength-power athletes (Grgic \& Mikulic, 2017; H. J. 
Pritchard et al., 2016). Therefore, experimenting with different tapering models for less important COMP for weightlifters may be beneficial to determine when and how the athlete is responding to the training stimulus.

\section{Tapering Practices for Weightlifting: A Brief Review}

Tapering allows a window of opportunity for the athlete to express peak performance and show adaptation acquired from the training program leading up to a COMP. The majority of the current tapering literature examines individual endurance sport athletes and endurance events (Mujika, 2009; Murach \& Bagley, 2015). There are two possible reasons for this: first, it is easy

to quantify physiological, psychological, and performance characteristics of a single athlete such as a swimmer, cyclist, or marathon runner. Second, these variables can be easily separated, isolated, and quantified without the multifaceted nature of sports team dynamics for physiological demands, training and performance changes (Mujika, 2009; Murach \& Bagley, 2015; Pyne et al., 2009). Team sports consist of a multitude of different positions, demands, and performance characteristics of each player that make up the entire team. Therefore, from a practical perspective, single subject designs and case studies on the top athletes in the world should be carried out in order to better understand how athletes at the highest level are able to perform, recover, adapt, and train over short and long-term periods during their career (Kinugasa, 2013).

Up to this point, peaking and tapering have been defined and characterized to a great extent provided by the current literature that exists on the topic. An understanding of how, when, and why to induce a taper after an overreach is vital in order to maximize performance for athletes, 
particularly in individual strength-power sports such as weightlifting. Until recently, tapering for individual barbell strength and power sports have received little attention. Thus, the primary focus of this section of the review is to summarize the known tapering practices for weightlifters within the confines of the current literature.

\section{$\underline{\text { Characteristics of Athletes }}$}

A brief description of the athletes used in these observations will be useful in order to make these findings relevant and practical for weightlifting athletes. In these weightlifting studies (Bazyler et al., 2018; Hornsby et al., 2017; Stone et al., 1996) a total of 18 athletes were observed with 5 females $(22.3 \pm 1.7 \mathrm{y}, 67.8 \pm 1.5 \mathrm{~kg} ; 163.6 \pm 2.3 \mathrm{~cm})$ and 13 males $(22.7 \pm 3.3 \mathrm{y}$, $92.2 \pm 10.5 \mathrm{~kg} ; 175 \pm 3.7 \mathrm{~cm})$.

\section{Tapering Patterns}

These studies (Bazyler et al., 2018; Hornsby et al., 2017; Stone et al., 1996) exclusively used exponential tapers with slow decay and step tapers. For weightlifters, exponential tapers seem to be used most frequently for higher level athletes preparing for important COMP (Bazyler et al., 2018; Hornsby et al., 2017; Stone et al., 1996). However, the use of step tapers was noted but this method is typically used when an athlete is "training through" a less important COMP. Sinclair score and gender did not seem to dictate tapering prescription in these studies (Bazyler et al., 2018; Hornsby et al., 2017; Stone et al., 1996). 


\section{$\underline{\text { Pre-Taper Practice and Taper Duration }}$}

Typically, high-level athletes use an overreach that is implemented into the peaking phase before the actual reduced training period begins in order to promote a positive performance change at the end of the phase. If the overreach is applied too early or too late supercompensation may not occur at the appropriate time, subsequently affecting the athlete's COMP performance.

With an appropriately timed overreach and taper, a positive performance outcome will, theoretically, be expressed. Regardless of the tapering model used weightlifters tend to implement the overreach approximately $3.7 \pm 0.8$ weeks prior to the COMP (Bazyler et al., 2018; Hornsby et al., 2017; Stone et al., 1996). The highest intensities ( $\geq 90 \%)$ observed were 1 week out from the COMP despite the level of importance (Bazyler et al., 2018; Hornsby et al., 2017; Stone et al., 1996).

\section{$\underline{\text { Training Volume-Load Manipulations }}$}

Training load manipulations that induced positive performance changes ranged from reduction between $40-59 \%$ with an average reduction in VL of $47 \%$. It should be noted that a reduction of $71 \%$ was observed in one case which resulted in the weightlifter not meeting COMP goals with a lower total than previous less important COMP (Bazyler et al., 2018). However, it should also be noted that males tend to reduce VL to a smaller degree than females $40-43 \%$ and $47-76 \%$, respectively. Stone et al., (1996) indicated that COMP total improved by $17.5 \mathrm{~kg}$ in a long taper group (i.e., exponential taper) and $8 \mathrm{~kg}$ in the short taper group (i.e., step taper). However, the long taper group was initially stronger and had higher COMP totals and Sinclair 
scores than the short taper group. Training VL was not specifically noted in this study by Stone et al. (1996) but it was stated that in the long taper group training volume decreased and significantly higher relative intensity was used during the last 4-weeks of training for both groups. Interestingly, a study observing national level powerlifters reported that stronger powerlifters (i.e., Wilks score >350) used longer tapers than weaker powerlifters (i.e., Wilks score <350) (Grgic \& Mikulic, 2017; Pritchard et al., 2016). Although it has not been observed, perhaps allometrically scaled performance using Sinclair Total for weightlifters follow a similar trend and could be used accordingly to determine the effectiveness of different taper types.

Once the overreach and taper are completed athletes typically undergo 1-3 days of training cessation prior to the COMP. Only one study reported training cessation that lasted $2.5 \pm 1.5$ days before a COMP. This time frame is shorter than what has been observed in the current literature dealing with other strength-power barbell athletes. Powerlifters typically take $3.5 \pm 0.7$ days off

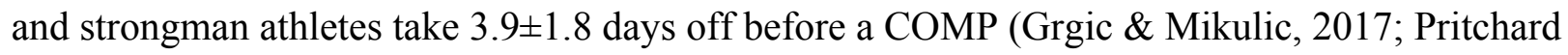
et al., 2016; Winwood et al., 2018). However, a recent study has suggested that training cessation of 3.5 and 5.5 days does not have significant outcomes on strength and power performance (Pritchard et al., 2018). Therefore, weightlifters may need shorter periods of training cessation because COMP movements require a high degree of motor control and neuromuscular skill (Raeder et al., 2017; Stone et al., 1993).

\section{Performance Change Expectations}

All of the aforementioned characteristics of the taper seem to influence the performance outcome, particularly the length of the taper and the reduction in VL. Typical taper lengths for 
weightlifters lasted approximately $19.8 \pm 6.5$ days. Exponential tapers are typically used for highlevel weightlifters when preparing for important COMP, but step tapers are also used for lesssignificant COMP where the athlete needs to achieve a relatively easily achieved qualifying total for a more important event. Total training VL reduced by an average of $47.3 \pm 8.3 \%$ which is still within the general tapering guidelines found within the current literature for strength-power athletes (30 to 70\%) (Pritchard et al., 2015). Also, the highest training volume loads or overreach

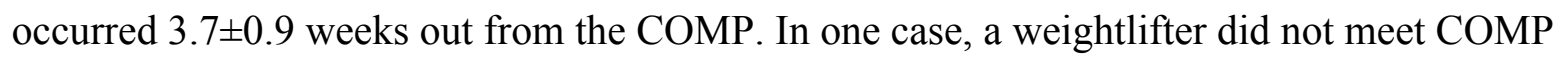
goals which could be due to weight cuts along with an excessive reduction in VL (Bazyler et al., 2018). The reduction in VL (i.e., 71\%) for the most important COMP fell outside the normal reduction parameters (i.e., $59 \%$ and $47 \%$, respectively) where the athlete was successful in obtaining competition goals. However, from the performance perspective, absolute and relative weightlifting performance increased for all weightlifting subjects within each study, respectively. Although descriptive in nature, these studies provide insight into how weightlifters prepare for COMP.

\section{Conclusion}

The intent of this review was to define and characterize tapering, examine psychological and physiological changes that may contribute to performance outcomes by implementing a taper and outline the magnitude of performance changes utilizing a taper after an overreach preparing for a COMP. Sport scientist and coaches should recognize that performance outcomes are not only influenced by COMP specific task but also a summation of psychological and physiological stress and adaptations that occur throughout the training process. It is vital to remember that the taper during a COMP phase is not aimed at achieving further adaptations but instead to reduce 
accumulated fatigue from previous training blocks. Mastering the principles of tapering as outlined throughout this review should not be the end goal. Instead, understanding and knowing how, when, and why to apply these principles to achieve peak performance may aid in accomplishing higher performance outcomes. The body of literature that has been reviewed may provide assistance for planning peaking-tapering protocols appropriately for weightlifters preparing for a major COMP. This study contributes to filling the gap between weightlifters and the paucity of peaking-tapering literature for strength-power athletes. Furthermore, monitoring psychological, physiological, and surrogate performance changes related to weightlifting ability should be used throughout the training process so that the athlete can recover, adapt, and unmask preparedness at its peak on the day of COMP. 


\title{
CHAPTER 3
}

PREPARING FOR A NATIONAL WEIGHTLIFTING CHAMPIONSHIP: A CASE SERIES

${ }^{1}$ S. Kyle Travis, ${ }^{1}$ Satoshi Mizuguchi, ${ }^{1}$ Michael H. Stone, ${ }^{2}$ William A. Sands, ${ }^{1}$ Caleb D. Bazyler

${ }^{1}$ Exercise and Sport Sciences, East Tennessee State University, Johnson City, TN, USA

${ }^{2}$ United States Ski and Snowboard Association, Park City, UT, USA

\author{
S. Kyle Travis, BS, CSCS - Corresponding Author \\ Department of Exercise and Sport Science \\ East Tennessee State University \\ PO BOX 70671 \\ Johnson City, Tennessee, 37614-1701 \\ Email: travissk@etsu.edu \\ Phone: 601-569-9588
}

Word Count 6,564 


\begin{abstract}
This study was an investigation of the time course of psychological, physiological and performance changes of two high-level weightlifters preparing for a major national weightlifting championship. A national level female $(24.5 \mathrm{y}, 53.8 \pm 0.3 \mathrm{~kg}, 155.4 \mathrm{~cm})$ and male weightlifter $(25.8 \mathrm{y}, 92.7 \pm 1.2 \mathrm{~kg}, 189 \mathrm{~cm})$ were recruited for the study. Body mass, hydration, psychological inventories, serum biomarkers, vastus lateralis muscle size, and jumping performance were assessed weekly beginning at 11-weeks from the competition date. In the final 30 days, each athlete used the same tapering protocol mode where training volume load was reduced by $48 \%$ for each athlete. Weightlifting performance goals were met for the female athlete (actual total $=159 \mathrm{~kg}$ ) but not for the male athlete (actual total $=292 \mathrm{~kg}$ ). For both athletes, it is almost certain that extremely large (likelihood $=99 \%$, effect size $[\mathrm{ES}]=4.7)$ and possible $(69 \%, \mathrm{ES}=0.4)$ body mass reductions took place the day of competition, respectively. Vastus lateralis muscle thickness $(53 \%, \mathrm{ES}=0.3)$ and cross-sectional area $(\mathrm{CSA})(65 \%, \mathrm{ES}=0.4)$ possibly decreased the week of competition for the female athlete, whereas CSA possibly decreased $(55 \%, \mathrm{ES}=0.4)$ the week of competition for the male athlete. The female and male athlete reported small positive changes for subjective recovery $(\mathrm{ES}=0.7 ; \mathrm{ES}=0.4$, respectively) along with a small and large positive change $(\mathrm{ES}=0.5 ; \mathrm{ES}=2.0)$ in stress levels. For the female and male athlete, respectively, serum hormones total testosterone $(81 \%, \mathrm{ES}=0.9 ; 81 \%, \mathrm{ES}=0.6)$, cortisol $(67 \%, \mathrm{ES}=0.8 ; 100 \%$, $\mathrm{ES}=35.5)$, testosterone-to-cortisol ratio $(63 \%, \mathrm{ES}=0.7,97 \%, \mathrm{ES}=0.6)$, sex-hormone-bindingglobulin $(78 \%, \mathrm{ES}=1.7 ; 49 \%, \mathrm{ES}=0.3)$ were inconsistent. Further, high sensitivity C-reactive protein $(99 \% \mathrm{ES}=3.0 ; 99 \%, \mathrm{ES}=7.6)$ and creatine kinase $(99 \%, \mathrm{ES}=0.2 ; 100 \%, \mathrm{ES}=3.6)$ was not reduced leading into the competition as expected for the female or male athlete, respectively. Unloaded squat jump height was peaked on competition day $(99 \%, \mathrm{ES}=2.7 ; 82 \%, \mathrm{ES}=2.1)$ for
\end{abstract}


both athletes. The taper may explain improved recovery and stress scores concurrent with jump height improvements. Psychological recovery and stress inventories paired with squat jumps may be used to assess high-level weightlifter's preparedness leading up to a major competition. Based on these observations and how each athlete responded to the taper, high-level male and female weightlifters may expect to achieve and maintain peak performance 3-4 days prior to a competition following a 1-week overreach and 3-week taper.

KEYWORDS: taper; peaking; jump height; weightlifting; snatch; clean and jerk 


\section{INTRODUCTION}

A properly structured training program for a high-level athlete aims at expressing preparedness and attaining the highest performance level possible at a major competition (COMP) within training and COMP macrocycle. Currently, in strength-power sports such as weightlifting, an optimal model for tapering has not been established and most sports scientists and coaches use a trial and error approach (Mujika \& Padilla, 2003; Thomas \& Busso, 2005). Thus, research investigating the time course of performance changes in response to tapering strategies used by weightlifters is needed. The aim of tapering is to reduce negative physiological and psychological residual effects from daily training (i.e., accumulated fatigue) rather than achieving further improvements regarding positive adaptions from training (i.e., fitness gain) (Mujika, 2010). Therefore, the final overreach leading into a COMP should be applied appropriately to ensure that training adaptations are not lost followed immediately by a taper to promote adequate recovery.

Accumulated fatigue from an increased training stimulus prior to tapering (i.e., overreach period) can remain elevated until the training volume-load (VL) is reduced. Ensuring that the training VL is reduced appropriately can be accomplished by monitoring changes in endogenous steroid hormone concentrations relative to baseline concentrations. In the current literature, totaltestosterone (T), cortisol (C), testosterone-to-cortisol (T:C) ratio, and sex-hormone binding globulin (SHBG) has been observed in high-level weightlifters (Storey \& Smith, 2012; Viru \& Viru, 2001). For instance, the equilibrium amid anabolic and catabolic states in weightlifters can be represented by the $\mathrm{T}: \mathrm{C}$ ratio and fluctuations can be used to indicate the current level of training strain relative to training VL (Haff et al., 2008; Wu et al., 2008). For instance, an 
increase in $\mathrm{T}: \mathrm{C}$ ratio can be indicative of low training strain (i.e., lower VL) whereas a decrease in $\mathrm{T}: \mathrm{C}$ ratio shows high levels of training strain (i.e., increased VL). However, while some studies have demonstrated this association between T:C ratio and VL (Busso et al., 1992; Fry et al., 1993; Fry et al., 2000; Haff et al., 2008; Häkkinen et al., 1987; Wu et al., 2008) other studies have reported no substantial biochemical changes (Crewther, Heke, \& Keogh, 2011; Hartman et al., 2007) and attenuated biochemical changes in high-level weightlifters acquiring more training experience over a two year period (Fry et al., 1994; Kraemer et al., 1992). Further, blood-borne markers such as high-sensitivity C-reactive protein (hsCRP) and creatine kinase (CK) have been shown to reflect training-induced physiological strain in male and female weightlifters as well as strength trained athletes (Gentles et al., 2017; Hecksteden et al., 2016). The variability of physiological measurements from athlete to athlete pose a concern if all high-level weightlifters respond similarly or differently to training and more important to tapering.

Psychological changes can take place in stressful COMP environments coinciding with physiological and performance alterations. While elevated acute phase proteins and cytokines can be accurately measured after intense training, the athlete's subjective recovery and stress state may give additional insight into the athlete's psychological preparedness prior to competing (Hecksteden et al., 2016; Kellmann et al., 2018). A recent study showed that outside and COMP stressors highly influenced endocrine responses (i.e., T concentrations, C concentrations) and were associated with performance outcomes in high-level strength-power athletes (Siart et al., 2017). Similarly, psychological inventories of high-level female and male weightlifters have been previously observed suggesting that while some elite level athletes respond similarly, others have opposing responses (Mahoney, 1989). Thus, stressors at a major COMP for a high-level 
weightlifter could negate physiological or biochemical phenomenon taking place indicative of positive preparedness or those same stressors could promote success depending on how the athlete is able to cope with the COMP environment.

In addition to biochemical and psychological measures, relationships between muscular adaptations and weightlifting competition performance have also been examined. Studies have reported a relationship between muscle fiber properties and weightlifting performance (Fry et al., 2003; Scanlon et al., 2014), but for practical reasons, it is unrealistic to monitor these changes in weightlifters preparing for a national championship. Thus, ultrasonography can be used as a lessinvasive method to provide information to the sports scientist and coach about changes in muscle size during important periods prior to competing. Increases in vastus lateralis muscle crosssectional area (CSA) and muscle thickness (MT) have been observed following strength training (e.g., high intensity $\geq 85 \%$ 1-repetition maximum) in high-level strength-power athletes which has been attributed to the ability performance ability (Castro et al., 1995; Häkkinen et al., 1988). While high level weightlifters competing at national and international levels are generally muscular and lean, these athletes also have mature neural patterns that have been developed over years of training and furthering adaptations can be difficult to achieve (Brechue \& Abe, 2002; Häkkinen et al., 1988; Sale, 1988). Therefore, while monitoring changes in muscular size can be easily acquired, assessing an athletes neuromuscular function in accordance with training strain can be a difficult observation.

Considering the physiological and psychological changes in accordance with training strain, performance decrements can be noticed through secondary performance test that allows the 
athlete to express neuromuscular function. If the athlete is experiencing residual neuromuscular fatigue, decrements in performance will likely be expressed. Therefore, for an athlete to express neuromuscular performance characteristics (i.e., rate of force development [RFD]) to the greatest capacity, diminishing accumulated fatigue through a reduced training period is essential.

However, being tapering for strength-power athletes prior to a COMP is not clearly understood, reduced training may be advantageous for some athletes whereas reductions in VL too small or too large may lead to performance decrements in others.

Vertical jump performance can be a useful tool for predicting weightlifting talent due to biomechanical similarities between the activities (Garhammer, 1980, 1993; Garhammer \& Gregor, 1992). Vertical jump testing can be used to detect fluctuations in power output, jump height $(\mathrm{JH})$ and dynamic RFD capabilities throughout training cycles leading up to a COMP. Jump height, specifically squat-jump JH ( $\mathrm{SJH})$, is considered to be strongly related to absolute and scaled weightlifting performance despite sex and weightlifting ability and can be used as a correlate to monitor weightlifting performance in male and female weightlifters (Carlock et al., 2004; Travis et al., 2017; Vizcaya et al., 2009). Vertical jumps are a preferred monitoring tool for assessing weightlifting COMP preparedness due to limited interference with training and practical use outside of the laboratory.

While overreaching and tapering in weightlifting have been studied, it is not clear whether high-level weightlifters preparing for an important COMP respond in the same way as what has been observed in the previous studies. Knowledge of differences and similarities in high-level weightlifters from and to the normal expectations derived from the previous studies can provide 
an example of what might be expected in high-level weightlifters. Thus, the purpose of this study was to characterize the psychological, physiological and performance changes of two high-level weightlifters preparing for a major national COMP.

\section{METHODS}

\section{Experimental Approach to the Problem}

This study used a case-series approach to monitor high-level weightlifters peaking for a national COMP. The athletes were part of an on-going athlete monitoring program and were familiar with all tests performed. Athletes were instructed to refrain from training and stimulants during the preceding $\geq 24 \mathrm{~h}$ and to arrive at the laboratory in a fully rested, hydrated state prior to testing. The final training session prior to a testing session consisted of various pushing and pulling movements, as well as practicing the full COMP lifts (i.e., snatch, clean and jerk). Each athlete was given a dietary log to record food consumption 24 hours prior to the testing sessions that involved blood draws. The athletes visited the laboratory on 28 occasions at the same time of day (0600-0800): twenty-seven testing sessions prior to leaving for the COMP and one testing session after the COMP once the athletes returned. Additionally, testing was administered on 2 separate occasions outside of the laboratory: once the day after arriving at the COMP site and once on COMP day prior to competing for a total of 30 testing sessions. The first five testing sessions were used for baseline measurements that occurred during a reduced training week at the end of consecutive training blocks to establish the within-athlete variability for all dependent variables. While all five baseline testing sessions included full battery testing procedures, the first initial baseline testing session included anthropometric measurements and somatotyping of each athlete followed by the full battery testing protocol. Beginning 11 weeks prior to COMP, a 
full battery testing session was administered on the first day and a partial battery testing session was administered on the fourth day of every training week (Refer to Table 3.1). After arriving at the COMP site, testing occurred during the same times as laboratory testing. The female weightlifter competed 2 days and the male weightlifter competed 4 days after arriving at the COMP site. On each athlete's COMP day, testing was conducted within 1 hour after the weighin prior to the competition start time.

Table 3.1 Testing Session Protocols

\begin{tabular}{ccc}
\hline Baseline Testing Session & Full Battery Testing Session & Partial Battery Testing Session \\
\hline Anthropometric Measurements & Hydration & Hydration \\
Somatotyping & Body Mass & Body Mass \\
Full Battery Testing Session & SRSS & SRSS \\
& Blood Draw & Standardized Warm-Up \\
& Ultrasonography & Squat Jump \\
& Standardized Warm-Up & \\
& Squat Jump & \\
\hline
\end{tabular}

SRSS: Short Recovery Stress Scale; Standardized Warm-Up: forward walking lunges, backward walking lunges, alternating lateral lunges, straight leg march, and walking quad stretch over a distance of $10 \mathrm{~m}$ per movement followed by 10 repetitions of bodyweight squats. Anthropometric measurements included standing and seated height, body mass, sum of 7 skinfolds, and Heath-Carter somatotyping.

\section{Athletes}

Two USA national level weightlifters were recruited to participate in this study. During the recruitment phase, each subject was given verbal explanations identifying key aspects of the study and an informed consent document outlining the purpose of the study, methodology, and possible risk and benefits that may result from participation were explained. All of the athletes 
voluntarily agreed to participate in the study and signed an informed consent form approved by the university's Institutional Review Board.

Case 1 was an elite level female weightlifter $(23.5 \mathrm{y} ; 53.8 \pm 0.3 \mathrm{~kg} ; 155.4 \mathrm{~cm}$; accolades: $77 \mathrm{~kg}$ snatch, $98 \mathrm{~kg}$ clean and jerk [C\&J], $171 \mathrm{~kg}$ total, and 238 Sinclair score) who has represented Team USA placing in the top 10 at an international COMP as well as medaling at more than 10 national COMP. Case 2 is a national level male weightlifter $(25.8 \mathrm{y} ; 92.7 \pm 1.2 \mathrm{~kg} ; 189.5 \mathrm{~cm}$; accolades: $135 \mathrm{~kg}$ snatch, $169 \mathrm{~kg}$ C\&J, 304kg total and 347 Sinclair score) who has medaled at 3 national COMP and primarily competes in USA national level COMP consistently placing within the top 30 (Refer to Table 3.2). 
Table 3.2 Descriptive Characteristics

\begin{tabular}{|c|c|c|}
\hline \multirow[b]{2}{*}{ Variables } & \multirow{2}{*}{$\begin{array}{l}\text { Case 1: Female } \\
\text { Baseline }\end{array}$} & \multirow{2}{*}{$\begin{array}{l}\text { Case 2: Male } \\
\text { Baseline }\end{array}$} \\
\hline & & \\
\hline \multicolumn{3}{|l|}{ Anthropometrics } \\
\hline Standing Height $(\mathrm{cm})$ & 155.4 & 189.0 \\
\hline Mass (kg) & $53.8 \pm 0.3$ & $92.7 \pm 1.2$ \\
\hline Somatotype & $2.2-4.8-1.7$ & $2.3-6.5-2.0$ \\
\hline \multicolumn{3}{|l|}{ Performance } \\
\hline Snatch (kg) & 77 & 135 \\
\hline Clean and Jerk (kg) & 98 & 169 \\
\hline Total (kg) & 171 & 304 \\
\hline Sinclair (A.U.) & 238 & 347 \\
\hline $\mathrm{SJH}(\mathrm{cm})$ & $30.3 \pm 1.3$ & $47.0 \pm 0.4$ \\
\hline RFD (N/s) & $2967.3 \pm 238.5$ & $6279.3 \pm 924.9$ \\
\hline \multicolumn{3}{|l|}{ SRSS } \\
\hline Recovery Score & $5.4 \pm 0.8$ & $4.7 \pm 0.3$ \\
\hline Stress Score & $1.4 \pm 1.3$ & $1.4 \pm 0.5$ \\
\hline \multicolumn{3}{|l|}{ Biomarkers } \\
\hline $\mathrm{T}(\mathrm{ng} / \mathrm{dL})$ & $171.0 \pm 13.0$ & $366.0 \pm 24.3$ \\
\hline C (ug/dL) & $18.9 \pm 1.5$ & $15.7 \pm 2.8$ \\
\hline T:C Ratio (A.U.) & $9.1 \pm 0.5$ & $2.4 \pm 0.5$ \\
\hline SHBG (nmol/L) & $77.0 \pm 5.5$ & $20.0 \pm 4.9$ \\
\hline hsCRP (mg/L) & $0.5 \pm 0.2$ & $0.2 \pm 0.0$ \\
\hline CK (U/L) & $74.2 \pm 18.4$ & $142.2 \pm 60.8$ \\
\hline \multicolumn{3}{|l|}{ Muscle Architecture } \\
\hline MT (cm) & $2.36 \pm 0.03$ & $2.74 \pm 0.02$ \\
\hline $\operatorname{CSA}\left(\mathrm{cm}^{2}\right)$ & $28.85 \pm 0.99$ & $40.69 \pm 0.71$ \\
\hline $\begin{array}{l}\text { Values are means } \pm \text { SD. } \\
\text { Somatotype score repres } \\
\text { SJH: squat jump jump-h } \\
\text { during SJ testing; T: tota } \\
\text { SHBG: sex-hormone-bin } \\
\text { creatine kinase; MT: mu }\end{array}$ & $\begin{array}{l}\text { mance metrics are tl } \\
\text { y Endo-Meso-Ecto } \\
\text { ecording from flight } \\
\text { sterone; C: cortisol; } \\
\text { lobulin; hsCRP: hig } \\
\text { ckness; CSA: cross }\end{array}$ & $\begin{array}{l}\text { accolades in competition. } \\
\text { e Heath-Carter method. } \\
\text { te of force development } \\
\text { osterone-to-cortisol ratio; } \\
\text {-reactive protein; CK: }\end{array}$ \\
\hline
\end{tabular}

\section{Training}

The athletes trained for 28-weeks using a block periodization (i.e., phase potentiation) model consisting of sequenced phases: strength, power, overreach and taper. The contest preparation phase of training began 74 and 76 days (i.e., approximately 11 weeks) out from the COMP for the female and male, respectively, which included 6-7 training sessions per week with increased 
intensity for traditional movements (e.g., back squat, overhead press, stiff-legged deadlift, etc.) and a marked increase for the COMP lifts (i.e., snatch and C\&J) (Refer to Table 3.3 and Table 3.4). Training goals for the major COMP for the female athlete and male athlete were to total $159 \mathrm{~kg}$ and $300 \mathrm{~kg}$, respectively. For the female weightlifter and the male weightlifter, each athlete used a 4-week peaking block consisting of an overreach during week 1 and training taper from week 2 through week 4 leading up to the COMP (Refer to Figure 3.1). Further, to quantify training for each athlete, training VL was determined by the sum of all training movements calculated by the following formula: training volume load $=$ load $(\mathrm{kg}) \cdot \operatorname{reps} \cdot$ sets.

Table 3.3 Final Training Block Leading into Competition for Case 1: Female Weightlifter

\begin{tabular}{cccc}
\hline Week 1 (Overreach) & Week 2 (Taper 1) & Week 3 (Taper 2) & Week 4 (Taper 3) \\
\hline Monday/Thursday & Monday/Thursday & Monday/Thursday & Monday/Thursday \\
\hline AM & AM & AM & AM \\
Squats & Squats & Squats & Jerk \\
PM & PM & PM & DB Press \\
Jerk & Jerk & Jerk & \\
Front Squat & Front Squat & Snatch Balance & \\
Snatch Balance & Snatch Balance & DB Press & \\
DB Press & DB Press & & \\
\hline Wednesday & Wednesday & Wednesday & Wednesday \\
\hline AM & AM & AM & AM \\
CGSS & CGSS & CGSS & CGSS \\
CG Pulls-PP & CG Pulls-PP & CG Pulls-PP & CG Pulls-PP \\
PM & PM & PM & PM \\
SGSS & SGSS & SGSS & SGSS \\
SG Pulls-Floor & SG Pulls-Floor & SG Pulls-Floor & SG Pulls-Floor \\
CG SLDL & CG SLDL & CG SLDL & CG SLDL \\
DB BOR & DB BOR & DB BOR & DB BOR \\
\hline Saturday & Saturday & Saturday & Saturday \\
\hline SGSS & SGSS & SGSS & COMP \\
Snatch & Snatch & Snatch & \\
C\&J & C\&J & C\&J & \\
SG SLDL & SG SLDL & SG SLDL & \\
DB BOR & DB BOR & DB BOR & \\
\hline
\end{tabular}

Note: $\mathrm{DB}=$ dumbbell, $\mathrm{CGSS}=$ clean grip shoulder shrugs, $\mathrm{CG}=$ clean grip, $\mathrm{PP}=$ power position, $\mathrm{SGSS}=$ snatch grip shoulder shrugs, $\mathrm{SG}=$ snatch grip, $\mathrm{SLDL}=$ straight legged deadlifts, BOR=bent over row. 
Table 3.4 Final Training Block Leading into Competition for Case 2: Male Weightlifter

\begin{tabular}{cccc}
\hline Week 1 (Overreach) & Week 2 (Taper 1) & Week 3 (Taper 2) & Week 4 (Taper 3) \\
\hline Monday/Thursday & Monday/Thursday & Monday/Thursday & Monday/Thursday \\
\hline AM & AM & AM & AM \\
Squats & Squats & Squats & Jerk \\
PM & PM & PM & BN Press \\
Front Squat & Jerk & Jerk & \\
Jerk & BN Press & BN Press & \\
BN Press & DB Press & DB Press & \\
DB Press & & & Wednesday \\
\hline Wednesday & Wednesday & Wednesday & AM \\
\hline AM & AM & AM & CGSS \\
CGSS & CGSS & CGSS & CG Pulls-Floor \\
CG Pulls-Floor & CG Pulls-Floor & CG Pulls-Floor & PM \\
PM & PM & PM & SGSS \\
SGSS & SGSS & SGSS & SG Pulls-PP \\
SG Pulls-PP & SG Pulls-PP & SG Pulls-PP & CG SLDL \\
CG SLDL & CG SLDL & CG SLDL & \\
Inverted Row & Inverted Row & Inverted Row & Saturday \\
\hline Saturday & Saturday & Saturday & COMP \\
SGSS & SGSS & SGSS & \\
Snatch & Snatch & Snatch & \\
C\&J & C\&J & C\&J & \\
SG SLDL & SG SLDL & SG SLDL & \\
Inverted Row & Inverted Row & Inverted Row & \\
\hline Note: DB $=$ dumbbell, CGSS=clean grip shoulder shrugs, CG=clean grip, PP=power position, \\
SGSS=snatch grip shoulder shrugs, SG=snatch grip, SLDL=straight legged deadlifts, \\
BOR=bent over row. & & &
\end{tabular}

\section{Testing Procedures}

\section{Anthropometrics and Hydration}

During the initial baseline assessment, standing and seated height were measured to the nearest 0.01 meters using a stadiometer (Cardinal Scale Manufacturing Co., Webb City, MO), body mass was measured using a digital scale (Tanita B.F. 350, Tanita Corp. of America, Inc., Arlington Heights, IL), and the sum of 7 skinfolds (chest, axilla, triceps, subscapular, abdominal, suprailium, thigh) was measured by an International Standards for Anthropometric Assessment 
(ISAK) certified anthropometrist. All skinfold measurements were collected from the right-side of the body in duplicate, or triplicate if the total error of measurements of the first and second measurement was $>5 \%$ of the mean value calculated (International Society for the Advancement of Kinathropometry, 2001). Accordingly, body fat percentage was estimated using a large number of regression equations and transformation data calculated from bone, muscle, adipose and residual masses using fractionation of body mass techniques calculated in an ISAK (2001) online published sheet. Other anthropometric variables were assessed to determine the somatotype of each athlete using the Heath-Carter method (1967): bicep and medial calf skinfolds, bicep girth (flexed $90^{\circ}$ and tensed), standing calf girth, abdominal and hip girth, biepicondylar femur and humerus breadth (Orvanová, 1990). For every testing session, each athlete's hydration state was estimated using a portable refractometer that calculates urine specific gravity (USG) on a scale ranging from 1.000 to 1.060 (Atago 4410 PAL-10S, Tokyo, Japan). If the refractometer $\mathrm{USG} \geq 1.020$, the athlete was instructed to drink water and hydration status was retested after 20 minutes. If the athlete failed $\mathrm{USG}<1.020$, further testing would not resume until the athlete demonstrated adequate hydration.

\section{Short Recovery and Stress Scale}

The short recovery and stress scale (SRSS) consists of 8 items related to recovery and stress (Kölling et al., 2015; Nässi, Ferrauti, Meyer, Pfeiffer, \& Kellmann, 2017; Pelka, Schneider, \& Kellmann, 2017). The adjectives can be grouped into four sub-scales relating to recovery and four sub-scales relating to stress. The recovery-related scales displayed one item for each subcategory: Physical Performance Capability, Mental Performance Capability, Emotional Balance, and Overall Recovery. The stress-related scales displayed one item for each sub- 
category: Muscular Stress, Lack of Activation, Negative Emotional State, and Overall Stress. Furthermore, each item is described by 4 adjectives, (e.g., Mental Performance Capability is described by the words attentive, receptive, concentrated, and mentally alert). The athletes were instructed to rate how much each expression applied to them prior to training and at the COMP, with responses on a Likert-type scale ranging from 0 (does not apply at all) to 6 (fully applies). The SRSS was completed a total of 30 times for each athlete. Previous studies have reported internal consistency of the recovery-related items of $a=0.70$ and $a=0.76$ for the stress-related items (Nässi et al., 2017; Pelka et al., 2017). Reliability and sensitivity to change relative to training have been shown to be adequate in various studies (Kölling et al., 2015; Nässi et al., 2017; Pelka et al., 2017).

\section{Biochemical Assessment}

All blood collection occurred between 0600-0800 following an overnight fast before the first training session of the week at least 48 hours after a training session or COMP. Blood was collected from the antecubital vein into a serum clot tube and was allowed to clot for 20 minutes at room temperature. The samples were centrifuged at $3400 \mathrm{rpm}$ for 15 minutes at room temperature. Serum was aliquoted into smaller centrifuge tubes and stored in a $-80^{\circ} \mathrm{C}$ freezer. Total-testosterone, C, SHBG, hsCRP, and CK were measured using an IMMULITE 1000 automated immunoassay analyzer (Siemens Healthcare, Erlangen, Germany) for each athlete. However, due to female testosterone levels typically falling below the lowest concentration threshold, $\mathrm{T}$ for the female athlete was measured using a solid-phase sandwich enzyme-linked immunosorbent assay (ELISA) (DSX ${ }^{\mathrm{TM}}$ Automated ELISA System 6.26, Dynex Technologies, Inc., Chantilly, VA). The $\mathrm{T}$ : $\mathrm{C}$ ratio was calculated by dividing the $\mathrm{T}$ concentration by the $\mathrm{C}$ 
concentration. Sample concentrations were determined by interpolating their respective absorbance values obtained from standard concentrations (Molecular Devices, Sunnyvale, CA). The CV calculated to determine intra-assay variability ranged from $0.49 \%$ to $12.3 \%$.

\section{Ultrasonography}

A 7.5 MHz ultrasound probe was used to measure MT and muscle CSA of the right vastus lateralis (LOGIQ, P6, General Electric Healthcare, Wauwatosa, WI). Each athlete laid in a left side recovery position with hips perpendicular to the examination table in the axial plane. Sampling location for the vastus lateralis was at $50 \%$ of the distance between the greater trochanter and the lateral epicondyle of the femur (Abe, Kumagai, \& Brechue, 2000). The location was marked with a permanent marker and the ultrasonography probe was covered with water-soluble transmission gel to aid acoustic coupling and avoid depression of the skin. Vastus lateralis MT was measured by placing the probe over the marked site and imaging was recorded. Vastus lateralis muscle CSA was measured by placing the probe perpendicular to the muscle and moving it in the transverse plane to collect the cross-sectional images. The mean of three images was used for analysis. Intra-session reliability of this method has been determined previously (Howe \& Oldham, 1996). For analysis, vastus lateralis MT was measured by drawing two lateral and one medial trace lines on the image between the superficial and deep aponeuroses. Muscle CSA was measured by tracing the inter-muscular interface around each muscle cross-sectional image. Intra-session reliability has been previously established for this measurement in our laboratory (intra-class correlation coefficient [ICC]: 0.99) (Bazyler et al., 2016). 


\section{Jumping Performance}

Following the standardized dynamic warm-up, unloaded squat jumps were performed on two dual axis force plates (PS-2142; PASCO Scientific, Roseville, CA, USA) affixed side by side connected to an interface (Airlink 2 PS-2010; PASCO Scientific) sampling at 1KHz using a personal laptop computer (MacBook Pro; macOS High Sierra v10.13.3; Apple, Cupertino, CA, USA). The squat jumps were performed with a near weightless plastic pipe placed across the shoulders similar to each athlete's back squat position. The tester instructed the athlete to perform a squat to a $90^{\circ}$ knee angle (i.e., ready position), which was measured using a handheld goniometer where the athlete held the position until the force-time trace was stable ( $\sim 2$ seconds). Once the force-time trace was stable with the lifter standing erect the tester instructed the athlete to get into the ready position. Once the force-time trace was stable in this position the tester shouted " $3,2,1 \ldots$ jump!" and the athlete performed a maximal effort jump. All jump trials were recorded with a customized template to record real-time jump height (PASCO Capstone software version 1.9.1; PASCO Scientific, Roseville, CA, USA). All jump trials were then exported to a custom jump analysis program (Lab View 2010, National Instruments Co., Austin, TX, USA). Squat jump (SJ) jump-height (JH) (SJH) was estimated from flight time which has been previously described in the literature (Linthorne, 2001). The mean of the best two trials within a $2 \mathrm{~cm}$ difference in $\mathrm{SJH}$ was used for analysis. Additional trials were performed if the difference between the two trials was greater than $2 \mathrm{~cm}$. Average RFD was determined from the beginning of the jump to the maximum force of the propulsion-acceleration phase. Intra-session reliability of this method has been previously established in our laboratory (ICC: 0.96-0.99) (Kraska et al., 2009) and average RFD reliability was established during this investigation (ICC: 0.96). 


\section{Statistical Analysis}

To evaluate meaningful changes throughout the training program the authors implemented the general guidelines for analysis of single-subject case study design as recommended by Kinugasa, (2013). The precision (i.e., probability, likelihood) of weekly changes relative to baseline values leading up to the COMP were calculated using a published online spreadsheet (Hopkins, 2000). Qualitative terms corresponding to the probability values associated with weekly changes relative to baseline values were classified as almost certainly not $(<1 \%)$, very unlikely $(<5 \%)$, unlikely $(<25 \%)$, possibly $(25-75 \%)$, likely $(>75 \%)$, very likely $(>95 \%$, and almost certain (>99\%). Effect sizes (ES) for each time point relative to baseline values were calculated for the mean difference between baseline and the subsequent time point value and then divided by the pooled standard deviation across the 5 baseline testing values (Hopkins, Marshall, Batterham, \& Hanin, 2009). Effect sizes were classified as trivial $(<0.3)$, small (0.3$0.9)$, moderate (0.9-1.6), large (1.6-2.5), very large (2.5-4.0), or extremely large (>4.0) (Hopkins, Marshall, Batterham, \& Hanin, 2009). The trivial region or smallest worthwhile change (SWC, ES $<0.3$ ) was used to determine whether changes were meaningful relative to baseline values (Hopkins, Hawley, \& Burke, 1999). All statistical analyses were calculated using Microsoft Excel 2016 (Microsoft Corporation, Redmond, WA, USA version 15.27).

\section{RESULTS}

\section{Volume-Load}

From the overreach week to the final taper week leading into the COMP, average training VL for the female athlete was reduced by $48 \%$, average training intensity (i.e., average load lifted) for traditional movements increased 5\%, and training intensity for the COMP lifts increased by 
$22 \%$ (Refer to Figure 3.1). For the male athlete, training VL was reduced by an average of $48 \%$, average training intensity for traditional movements remained the same $(<1 \%$ change), and intensity for COMP lifts increased by $4 \%$ (Refer to Figure 3.2).

Figure 3.1 Training volume load during the Competition Preparation Phase 11 weeks out from COMP date for the female weightlifter.

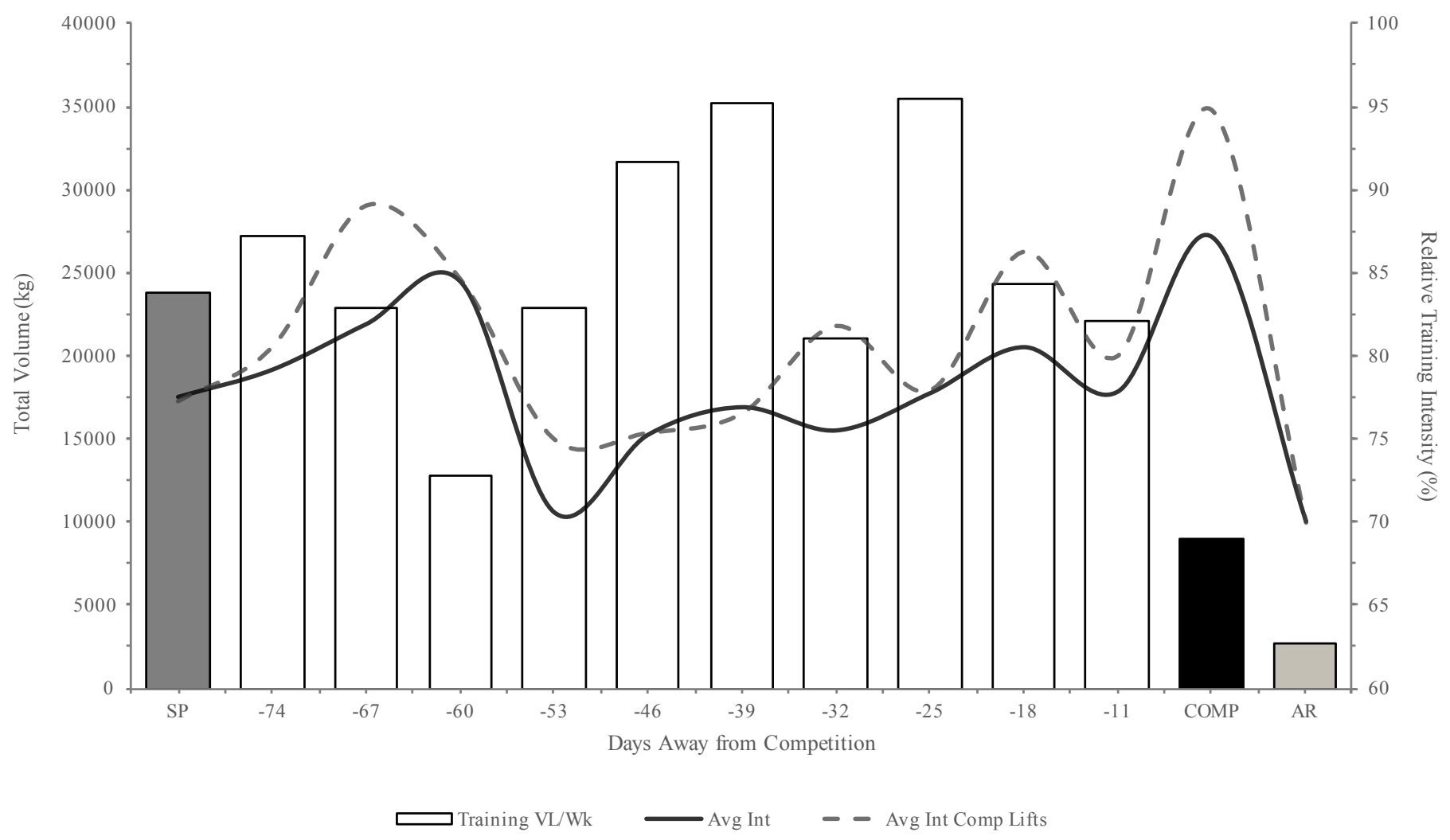

Note: (SP) Specific Preparation average training VL prior to competition phase. Competition Week (COMP). Active Recovery (AR) training after the COMP. Average intensity (Avg Int) consist of traditional training movements outside of the snatch and C\&J. Average intensity for the competitions lifts (Avg Int Comp Lifts) consist of snatch and C\&J only. 
Figure 3.2 Training volume load during the Competition Preparation Phase 11 weeks out from COMP date for the male weightlifter.

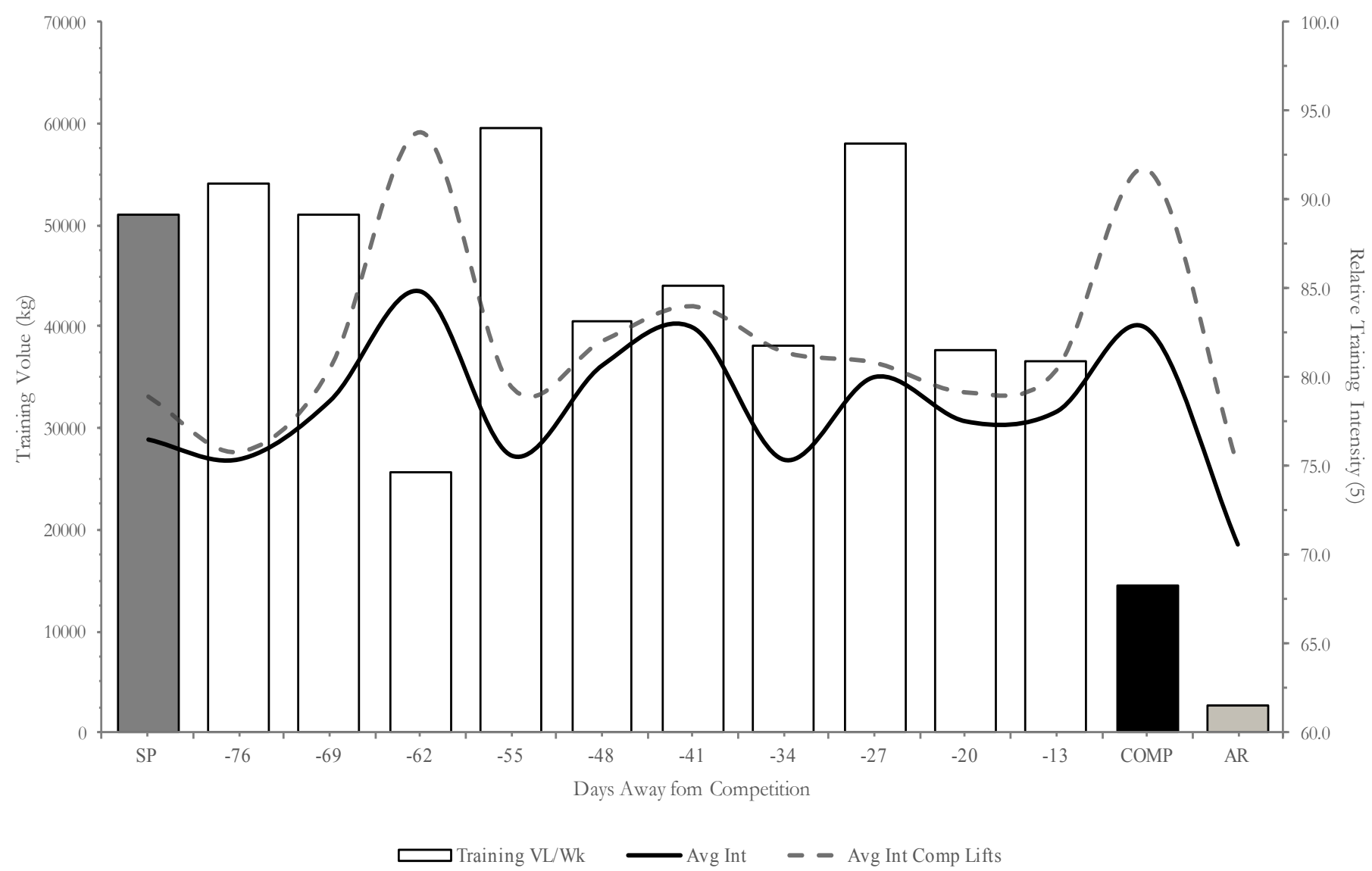

Note: (SP) Specific Preparation average training VL prior to competition phase. Competition Week (COMP). Active Recovery (AR) training after the COMP. Average intensity (Avg Int) consist of traditional training movements outside of the snatch and C\&J. Average intensity for the competitions lifts (Avg Int Comp Lifts) consist of snatch and C\&J only.

Weightlifting Performance, Anthropometrics, and Muscle Architecture

At the COMP, the female athlete met COMP goals (goal total $=159 \mathrm{~kg}$ vs actual total $=159 \mathrm{~kg}$ ). The male athlete did not meet COMP goals (goal total $=300 \mathrm{~kg}$ vs actual total $=292 \mathrm{~kg}$ ).

Nevertheless, the female athlete placed in the top 10 and the male athlete placed in the top 30 of each athlete's respective weight categories. 


\section{Case 1 Female}

In the first case, the elite-level female weightlifter was considered to be a mesomorphicendomorph (Endomorph, Mesomorph, Ectomorph Heath Carter scores: 2.2-4.8-1.7). During the tapering period, it was almost certain that extremely large body mass reductions (precision $=99 \%$; $99 \%$, ES=4.3; 4.7) took place beginning two days before and the day of COMP (Figure 3.3). Vastus lateralis MT and CSA possibly decreased $(53 \%, \mathrm{ES}=0.3 ; 65 \%, \mathrm{ES}=0.4)$ the week of COMP. However, further decreases in MT were almost certain (99\%, ES=1.7) following the COMP (Figure 3.4).

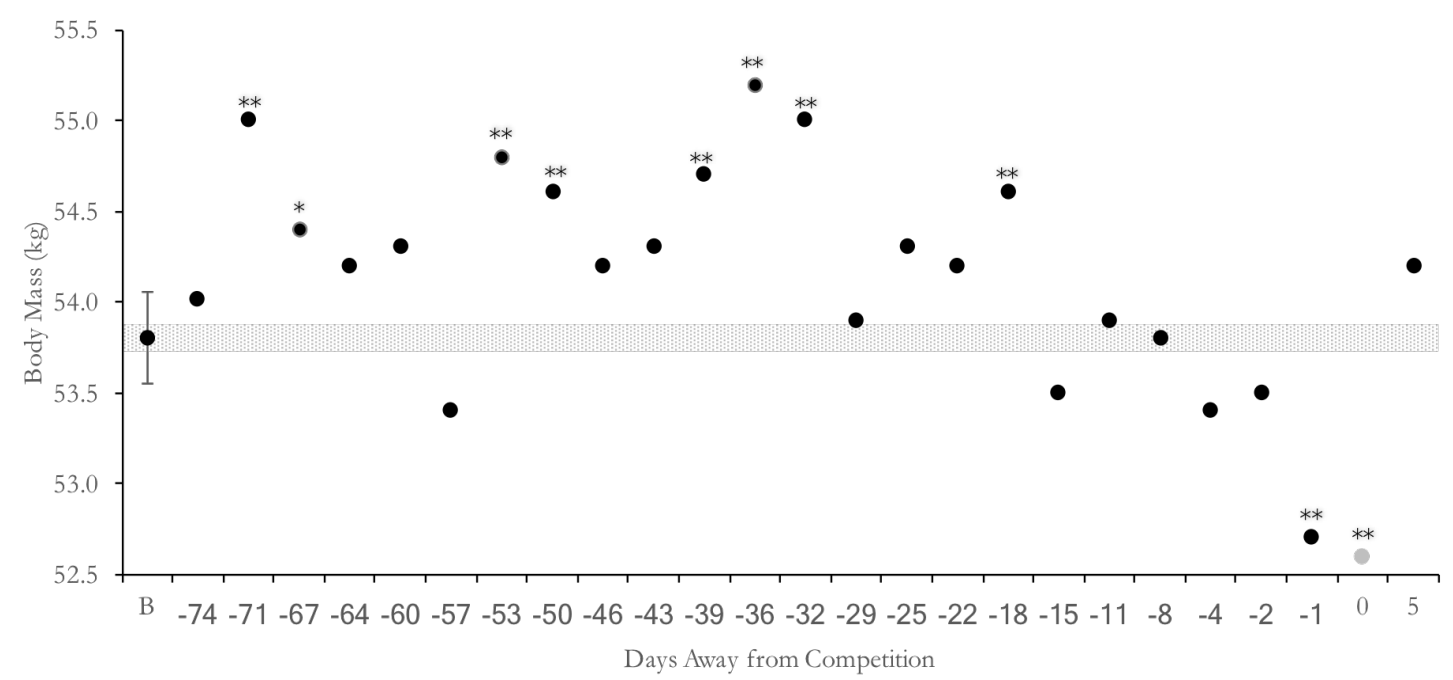

Figure 3.3 Body Mass changes during Competition Preparation Phase (female). Note: *indicates $>95 \%$ (very likely), ${ }^{* *}$ indicates $>99 \%$ (almost certainly). B: bassline testing sessions - average value during baseline measurements. Error bar is the standard deviation across baseline values. Gray region is the trivial or SWC. Gray marker above time point 0 is the day of competition. 


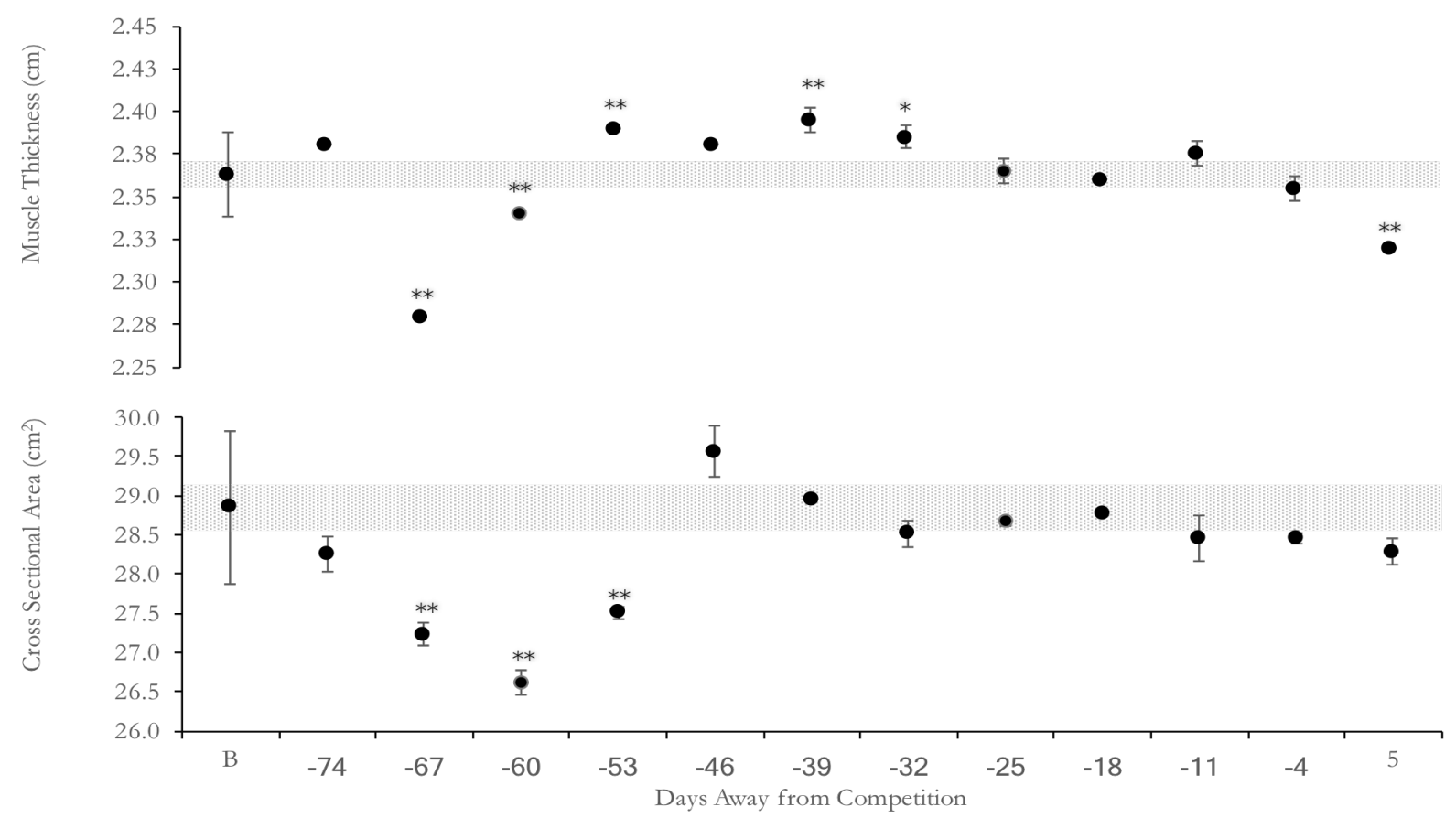

Figure 3.4 Muscle Thickness and Cross-Sectional Area changes during Competition Preparation Phase (female). Note: $*$ indicates $>95 \%$ (very likely), $* *$ indicates $>99 \%$ (almost certainly). B: bassline testing sessions - average value during baseline measurements. Error bar is the standard deviation across baseline values. Gray region is the trivial or SWC. Time point -4 is the week leading into the COMP.

\section{Case 2 Male}

In the second case, the national-level male was considered to be a mesomorphic-endomorph (2.3-6.5-2.0). During the overreach period, it was almost certain that large increases $(99 \%$, $\mathrm{ES}=2.1)$ in his body mass were noticed. However, possible small $(64 \%, 0.4)$ and trivial $(50 \%$, $\mathrm{ES}=0.3$ ) reductions were observed during the week of and after the COMP (Figure 3.5). It was very likely that moderate positive changes during the week of COMP (98\%, ES=1.1) and very large negative changes $(99 \%, \mathrm{ES}=4.0)$ in MT following the COMP took place. Muscle CSA possibly decreased (55\%, ES $=0.4)$ the week of COMP and further moderate reductions took place after the COMP (98\%, ES=1.3) (Figure 3.6). 


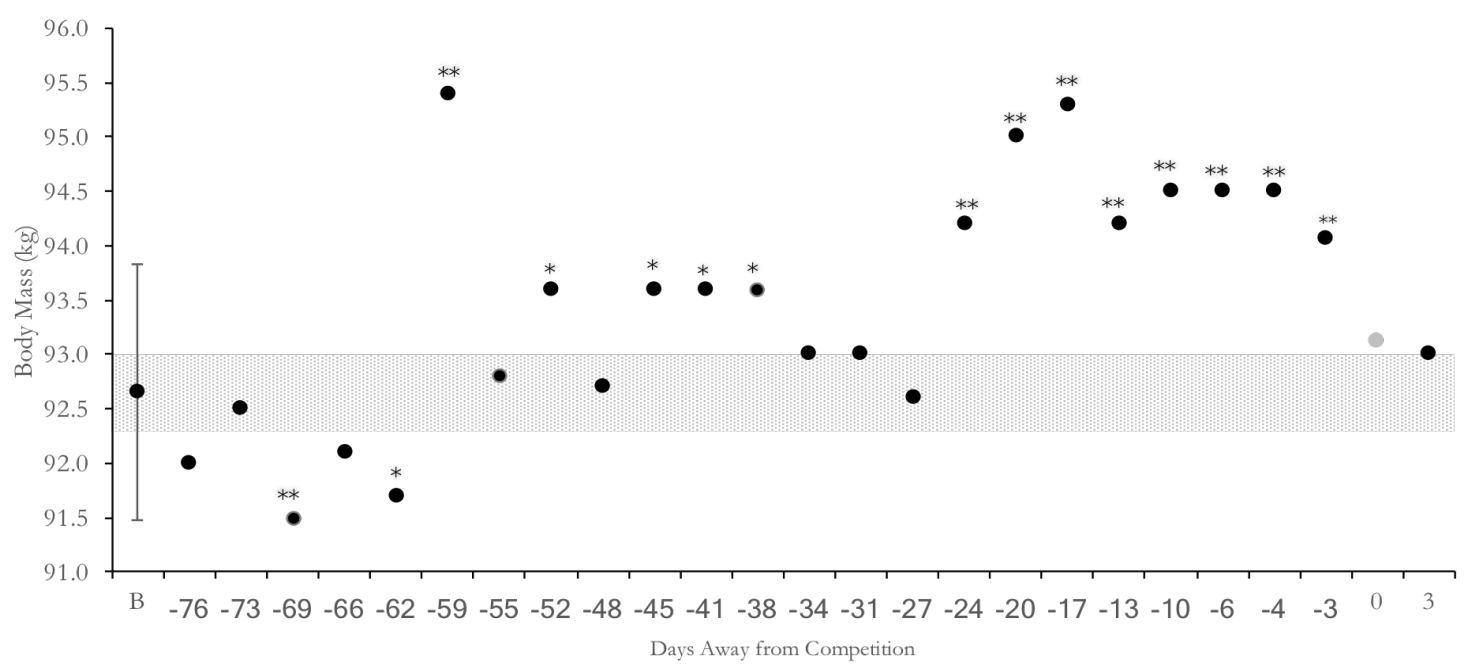

Figure 3.5 Body Mass changes during Competition Preparation Phase (male). Note: *indicates $>95 \%$ (very likely), ** indicates $>99 \%$ (almost certainly). B: bassline testing sessions - average value during baseline measurements. Error bar is the standard deviation across baseline values. Gray region is the trivial or SWC. Gray marker above time point 0 is the day of competition.

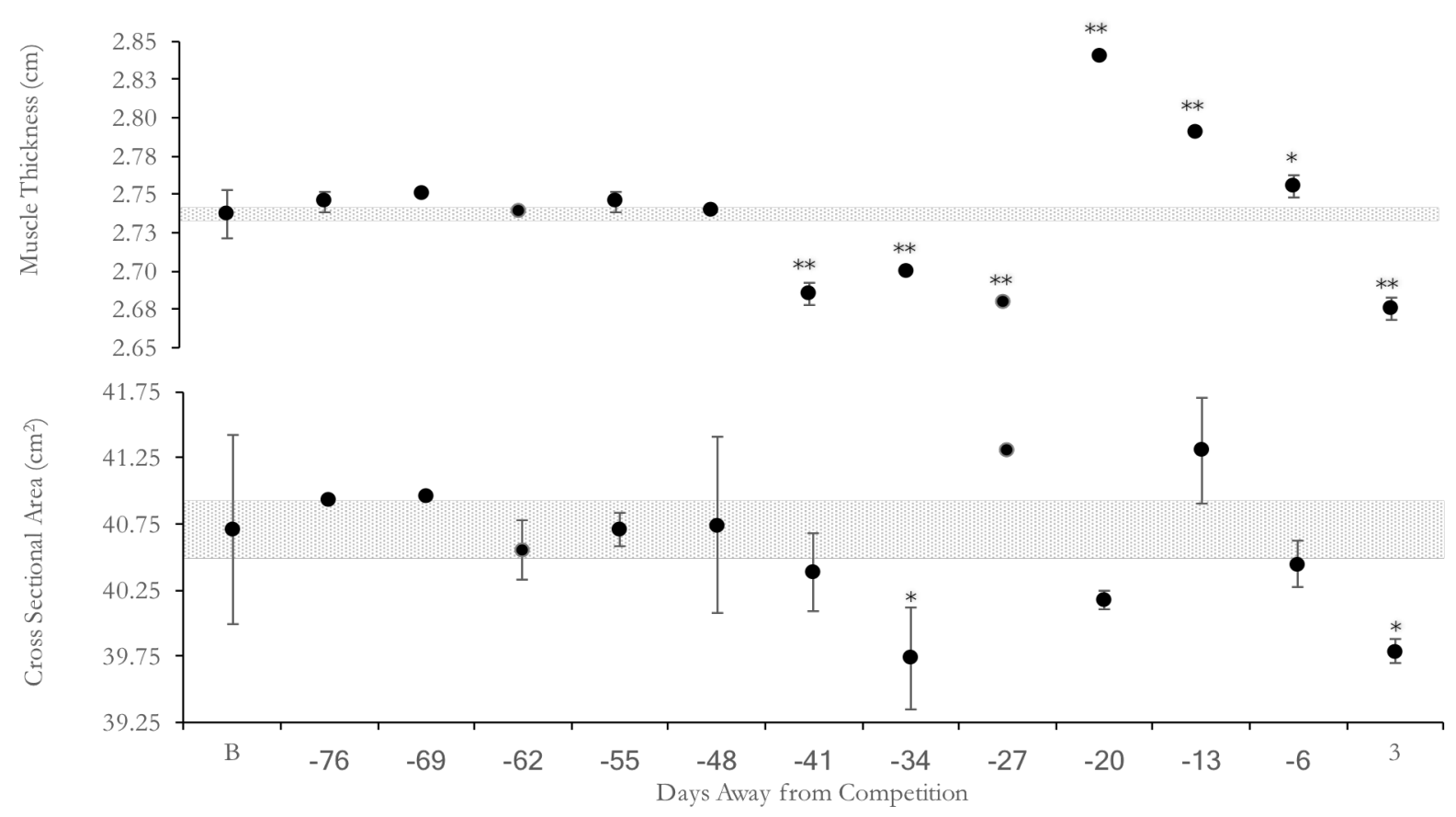

Figure 3.6 Muscle Thickness and Cross-Sectional Area changes during Competition Preparation Phase (male). Note: $*$ indicates $>95 \%$ (very likely), $* *$ indicates $>99 \%$ (almost certainly). B: bassline testing sessions - average value during baseline measurements. Error bar is the standard deviation across baseline values. Gray region is the trivial or SWC. Time point -6 is the week leading into the COMP. 


\section{Psychological changes}

Overall both athletes reported higher mean recovery scores relative to baseline with lower mean stress levels on the day of COMP (Figure 3.7 and Figure 3.8)

\section{Case 1 Female}

Throughout the study, the SRSS revealed the female athlete felt slightly less recovered (5.1 \pm 0.9$)$ with slightly increased stress $(1.7 \pm 1.3)$ relative to baseline values for recovery and stress (5.4 \pm 0.8 and $1.4 \pm 1.3$, respectively). The female athlete reported improved recovery (daily score $=6.0$ ) and lower stress (daily score $=0.8$ ) on the day of competition relative to baseline.

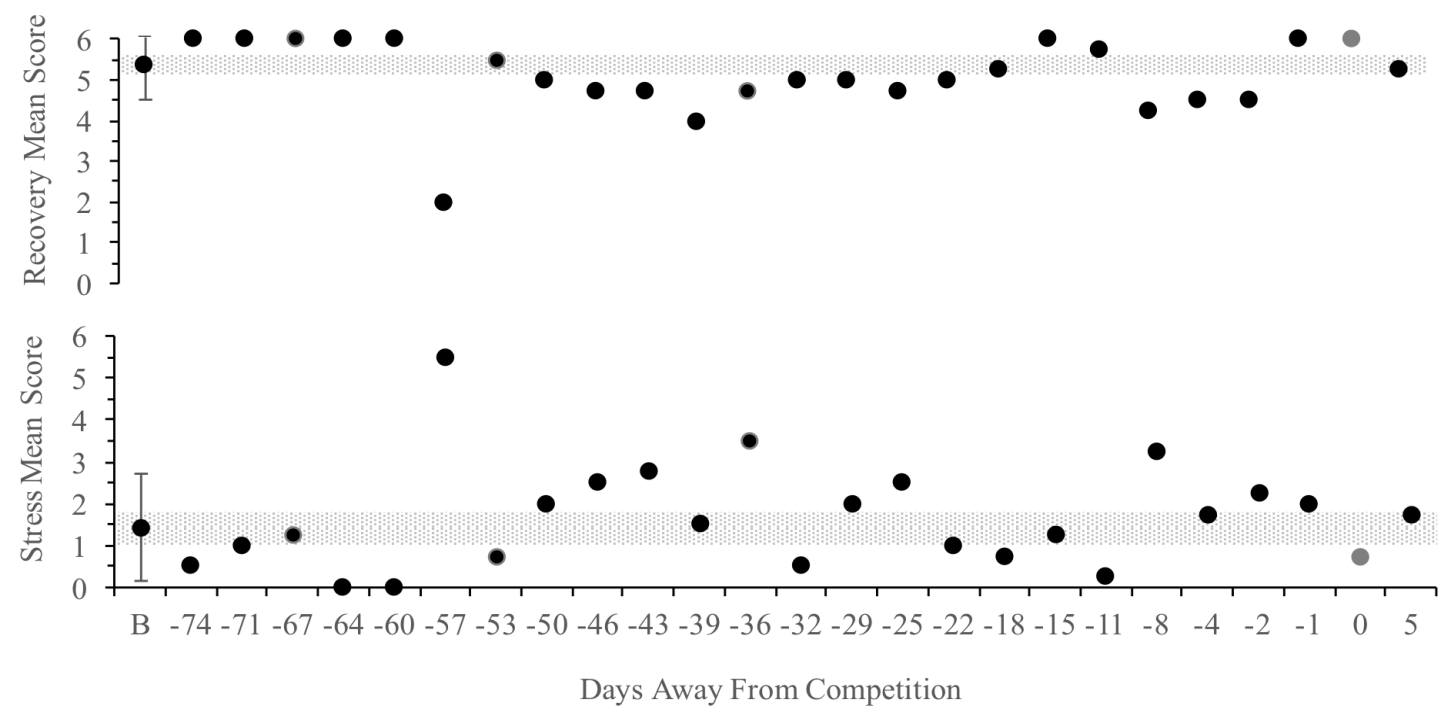

Figure 3.7. Short Recovery Stress Scale scores during Competition Preparation Phase (female). Note: B: bassline testing sessions - average value during baseline measurements. Error bar is the standard deviation across baseline values. Gray region is the trivial or SWC. Gray marker above time point 0 is the day of competition.

\section{Case 2 Male}

The male athlete showed signs of under-recovery (3.0 \pm 0.8$)$ with high-stress levels $(2.5 \pm 0.9)$ relative to baseline $(4.7 \pm 0.3$ and $1.4 \pm 0.5)$ for the duration of the study. However, on COMP day, 
the male athlete reported improved recovery (daily score=4.8) and lower stress levels (daily score $=0.5$ ) relative to baseline values.

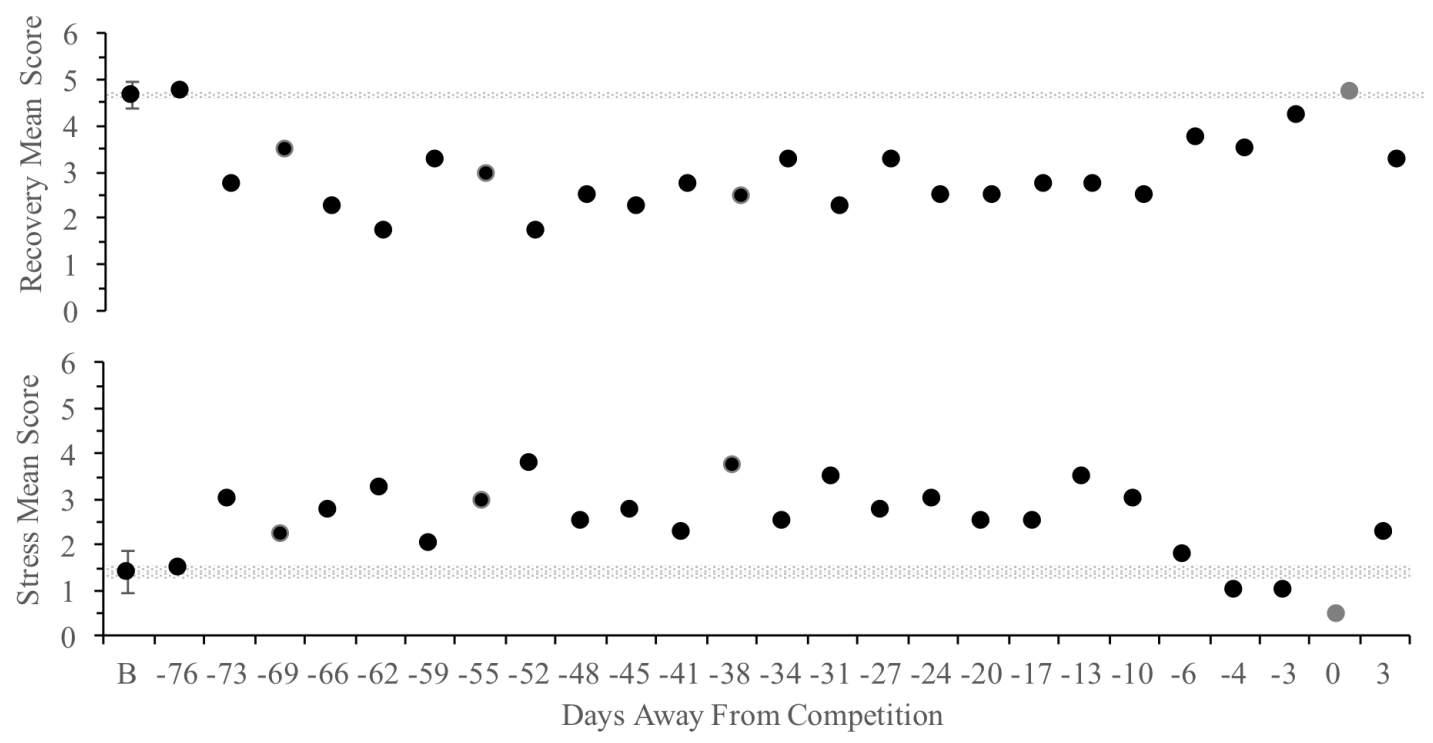

Figure 3.8 Short Recovery Stress Scale scores during Competition Preparation Phase (male). Note: B: bassline testing sessions - average value during baseline measurements. Error bar is the standard deviation across baseline values. Gray region is the trivial or SWC. Gray marker above time point 0 is the day of competition.

\section{Biochemical Changes}

\section{Case 1 Female}

Total testosterone almost certainly increased $(99 \%, E S=2.6)$ after the overreach and was very likely elevated moderately $(81 \%, \mathrm{ES}=0.9)$ the week of COMP and possibly reduced $(67 \%$, $\mathrm{ES}=0.6)$ below baseline following the COMP. Cortisol almost certainly increased $(99 \%, \mathrm{ES}=3.2)$ during week 1 of the taper and was possibly elevated (67\%, ES=0.8) during COMP week returning to baseline thereafter. Small reductions $(63 \%, \mathrm{ES}=0.7)$ in $\mathrm{T}: \mathrm{C}$ ratio were possibly observed after the COMP but remained within baseline values throughout the tapering period. Starting with the overreach, SHBG showed a positive trend up to the week of COMP where it is 
likely that large increases took place $(78 \%, \mathrm{ES}=1.7)$ with possible reductions following the COMP (73\%, ES=1.4) (Figure 3.9). Systemic inflammatory marker hsCRP almost certainly decreased $(99 \%, 99 \%, \mathrm{ES}=0.5$ and 0.6 , respectively) the week of and after the COMP. However, CK almost certainly showed no increases until after COMP where it was almost certainly elevated $(99 \%, \mathrm{ES}=0.4)$ (Figure 3.10).

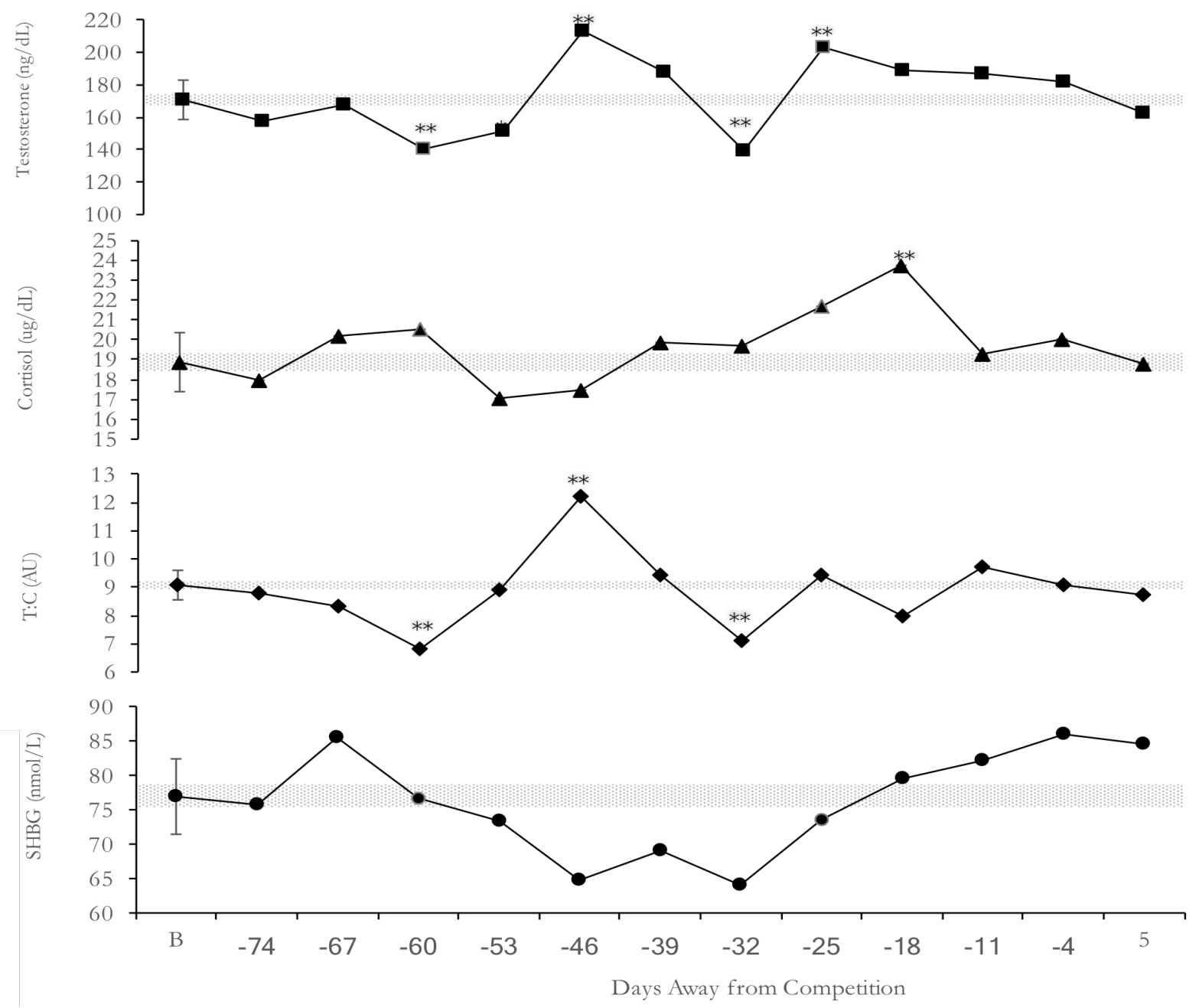

Figure 3.9 Endogenous Steroid Hormone changes during Competition Preparation Phase (female). Note: *indicates $>95 \%$ (very likely), $* *$ indicates $>99 \%$ (almost certainly). B: bassline testing sessions - average value during baseline measurements. Error bar is the standard deviation across baseline values. Gray region is the trivial or SWC. Time point -4 is the week leading into the COMP. 

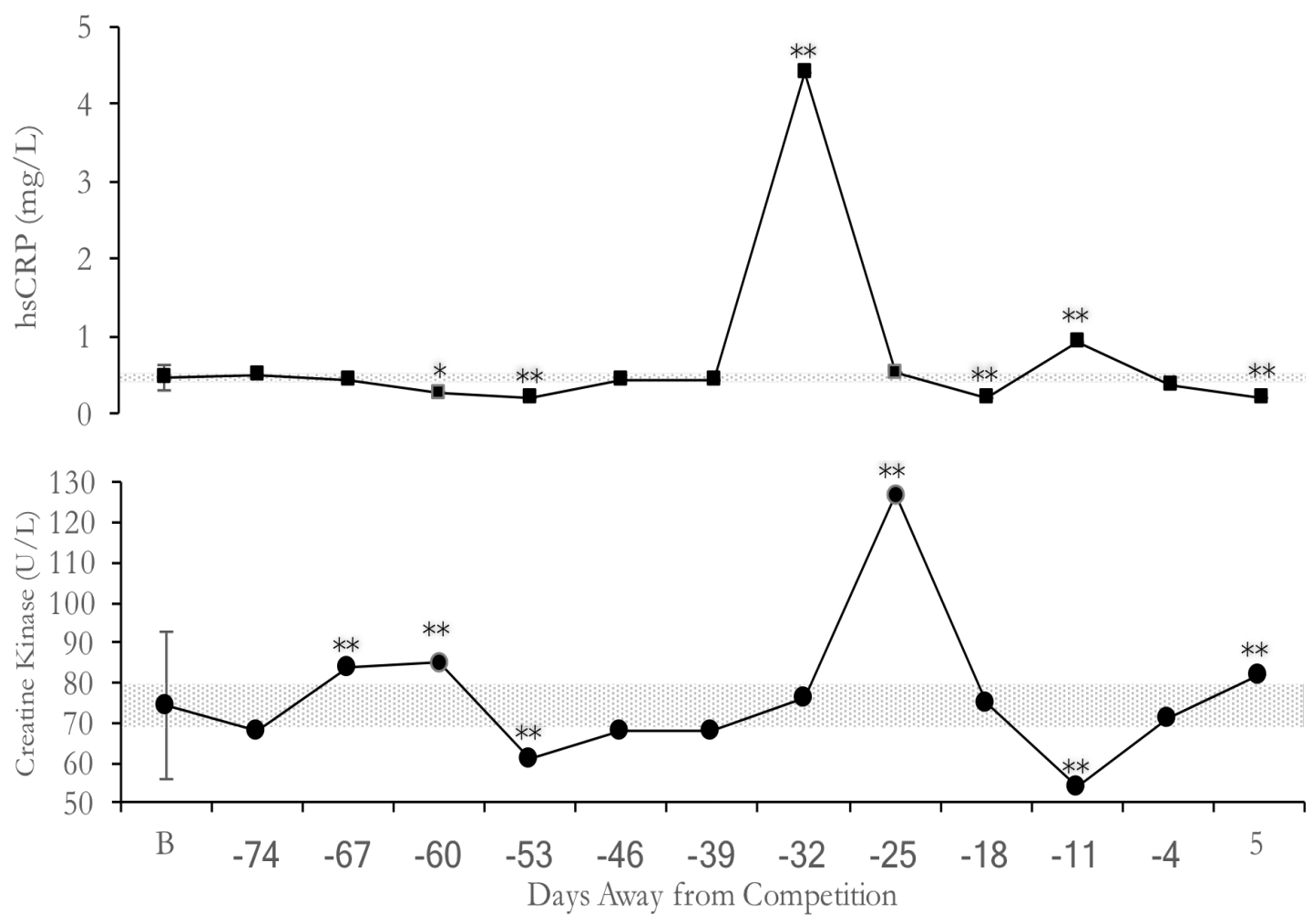

Figure 3.10 Inflammatory response during Competition Preparation Phase (female). Note: *indicates $>95 \%$ (very likely), ** indicates $>99 \%$ (almost certainly). B: bassline testing sessions - average value during baseline measurements. Error bar is the standard deviation across baseline values. Gray region is the trivial or SWC Time point -4 is the week leading into the COMP.

\section{Case 2 Male}

During the first week of the taper after the overreach, an extremely large reduction took place in $\mathrm{T}(100 \%$, ES=6.7). However, while $\mathrm{T}$ almost certainly increased $(99 \%, \mathrm{ES}=1.2)$ during the second week of the taper, a small reduction in $\mathrm{T}$ was likely observed the week of $(81 \%, \mathrm{ES}=0.6)$ and after $(94 \%, E S=0.9)$ COMP. Cortisol also showed a very large decrease during week 1 of the taper $(>99 \%, \mathrm{ES}=2.8)$ and an extremely large increase $(100 \%, \mathrm{ES}=35.3)$ week 3 of tapering. However, cortisol returned to baseline levels from the week of COMP and likely decreased $(80.7 \%, \mathrm{ES}=0.8)$ further after COMP. Testosterone-to-cortisol ratio almost certainly increased 
( $99 \%, \mathrm{ES}=3.6)$ week 2 of the taper, very likely decreased the week of COMP $(97 \%, \mathrm{ES}=0.6)$ and possibly may have increased following the $\mathrm{COMP}(67 \%, \mathrm{ES}=0.4)$. No changes were observed in SHBG relative to baseline with only possible trivial and small increases shown the week of (49\%, ES=0.3) and after COMP (51\%, ES=0.4) (Figure 3.11). An extremely large increase in hsCRP was observed the week of COMP (99\%, ES=7.6). For the week of and following COMP, a very large increase was also observed in CK levels $(100 \%, \mathrm{ES}=3.6 ; 100 \%, \mathrm{ES}=3.8 \%$, respectively) (Figure 3.12).

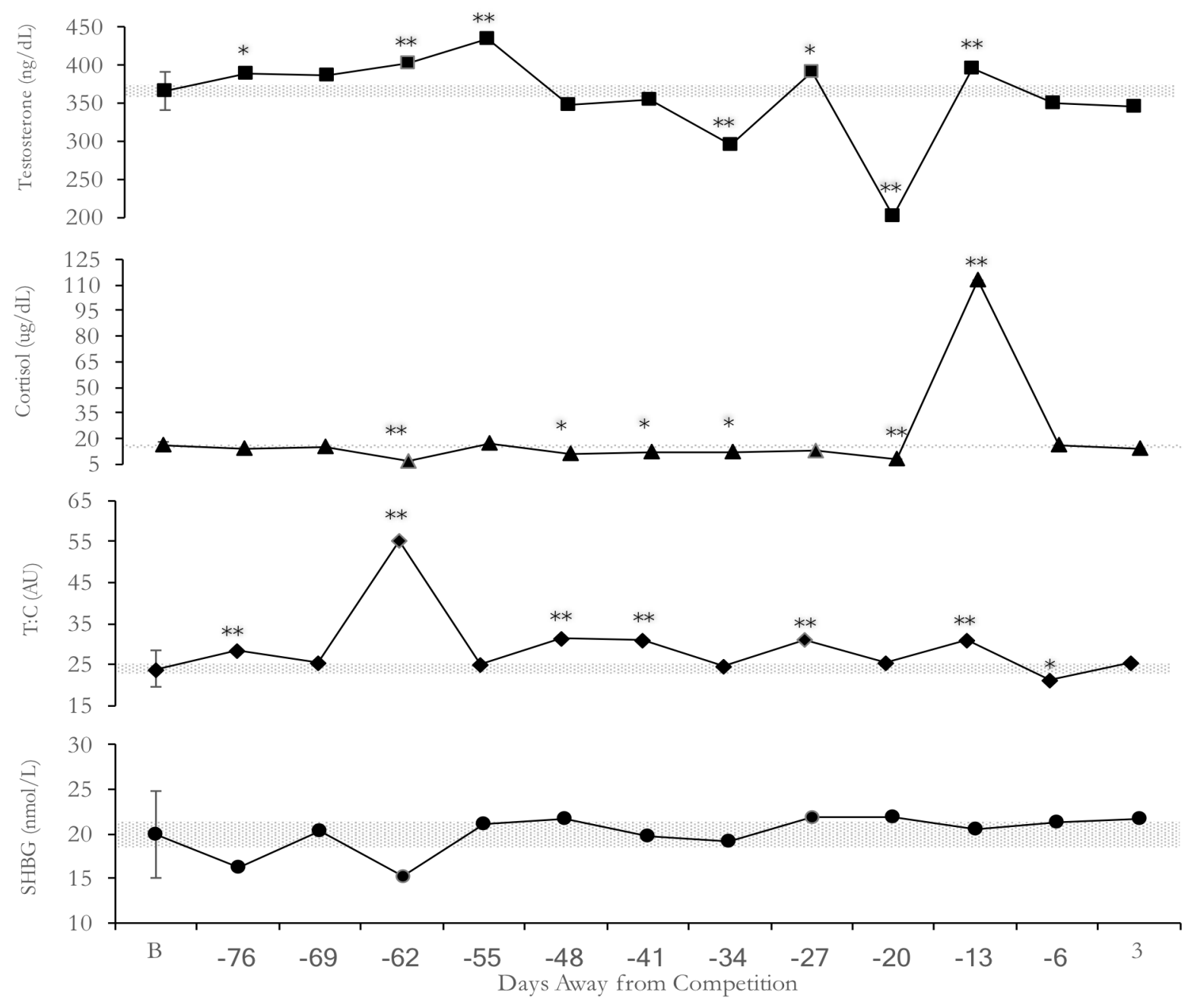

Figure 3.11 Endogenous Steroid Hormone changes during Competition Preparation Phase (male). Note: *indicates $>95 \%$ (very likely), ${ }^{* *}$ indicates $>99 \%$ (almost certainly). B: bassline testing sessions - average value during 
baseline measurements. Error bar is the standard deviation across baseline values. Time point -6 is the week leading into the COMP.
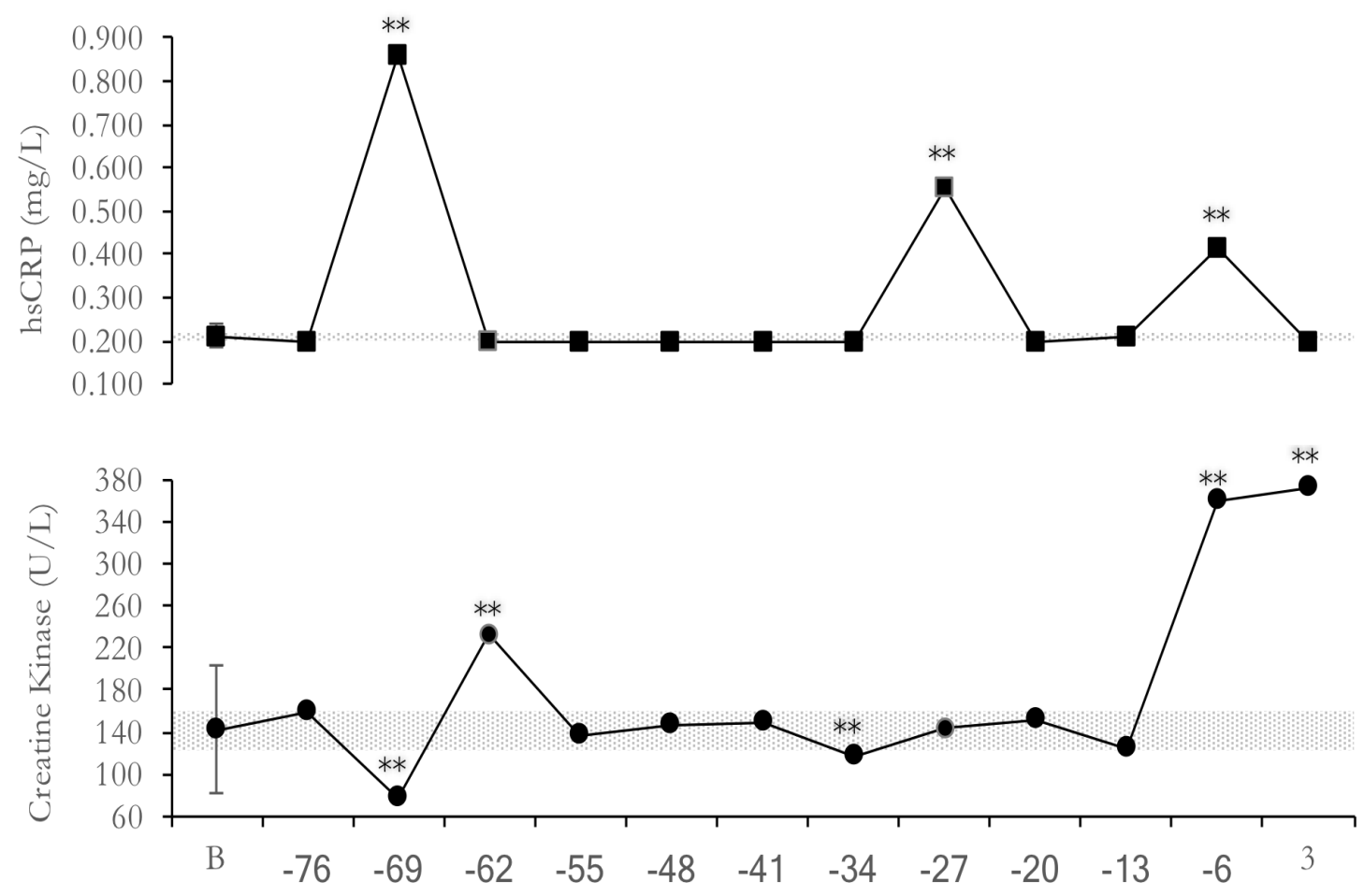

Figure 3.12 Inflammatory response during Competition Preparation Phase (male). Note: *indicates $>95 \%$ (very likely), ** indicates $>99 \%$ (almost certainly). B: bassline testing sessions - average value during baseline measurements. Error bar is the standard deviation across baseline values. Gray region is the trivial or SWC. Time point -6 is the week leading into the COMP.

\section{Jump Performance Changes}

Both athletes showed increases in jump height beginning 3 and 4 days prior to the COMP, on COMP day, and 3 and 5 days after the COMP, respectively.

\section{Case 1 Female}

For the female athlete, the day after arrival a moderate increase in SJH was likely (92\%, $\mathrm{ES}=1.1)$ and almost certainly peaked on the COMP day $(>99 \%, \mathrm{ES}=2.7)$. A likely large increase 
in RFD the day after arrival $(91 \%, E S=2.4)$ and very large increase likely occurred the day of COMP (96\%, ES=3.1) (Figure 3.13).
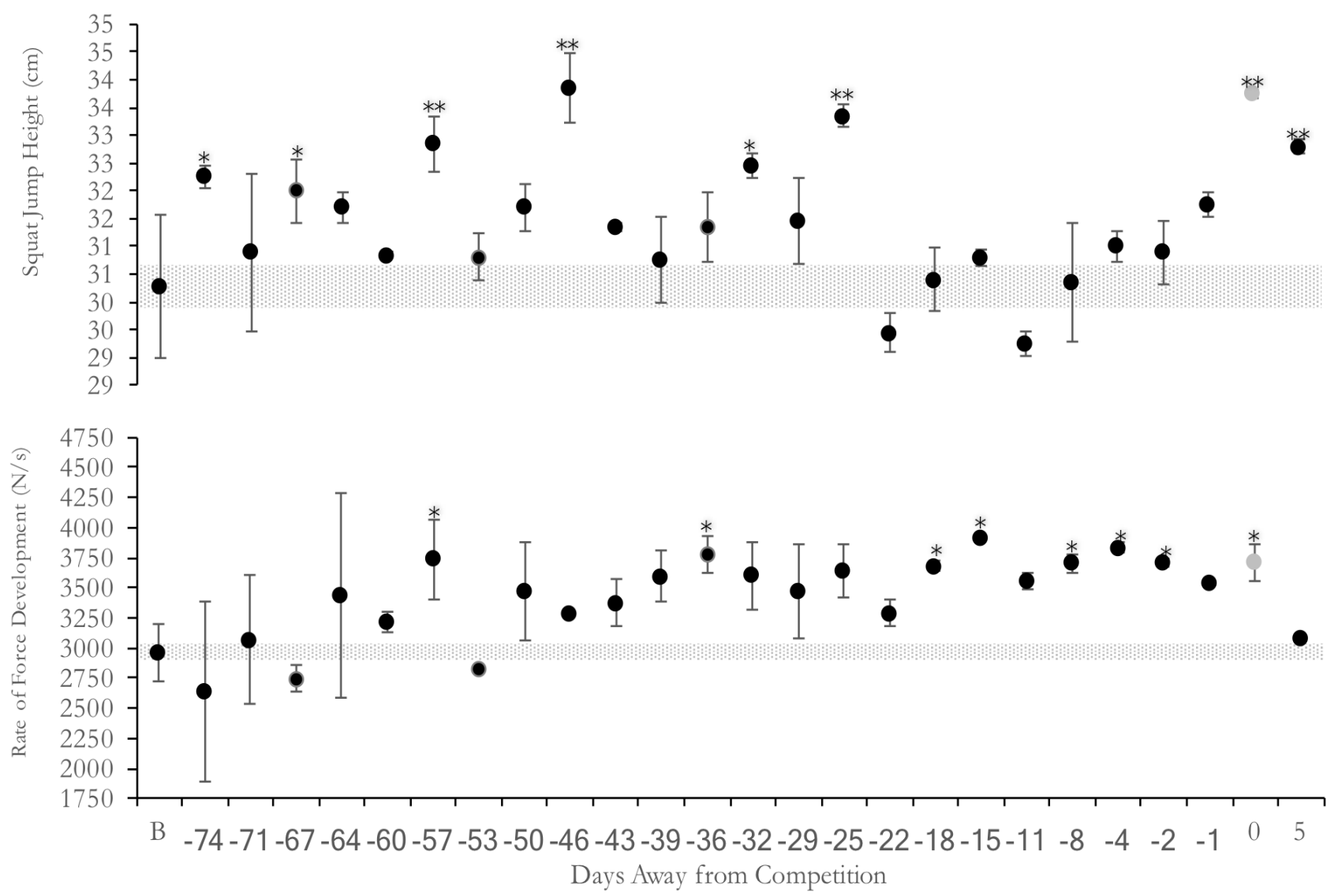

Figure 3.13 Jumping performance during Competition Preparation Phase (female). Note: *indicates $>95 \%$ (very likely), ** indicates $>99 \%$ (almost certainly). B: bassline testing sessions - average value during baseline measurements. Error bar is the standard deviation across baseline values. Gray region is the trivial or SWC. Gray marker above time point 0 is the day of competition.

\section{Case 2 Male}

Observations for $\mathrm{SJH}$ for the male athlete showed a very likely large increase the day after arrival $(91 \%, \mathrm{ES}=3.0)$, a likely large increase on COMP day $(82 \%, \mathrm{ES}=2.1)$, and very large probable increase after COMP took place $(94 \%, \mathrm{ES}=3.5)$. Rate of force development was almost certainly increased the day after arrival (99\%, ES=3.3) and on COMP day (99\%, ES=2.1) (Figure 3.14). 

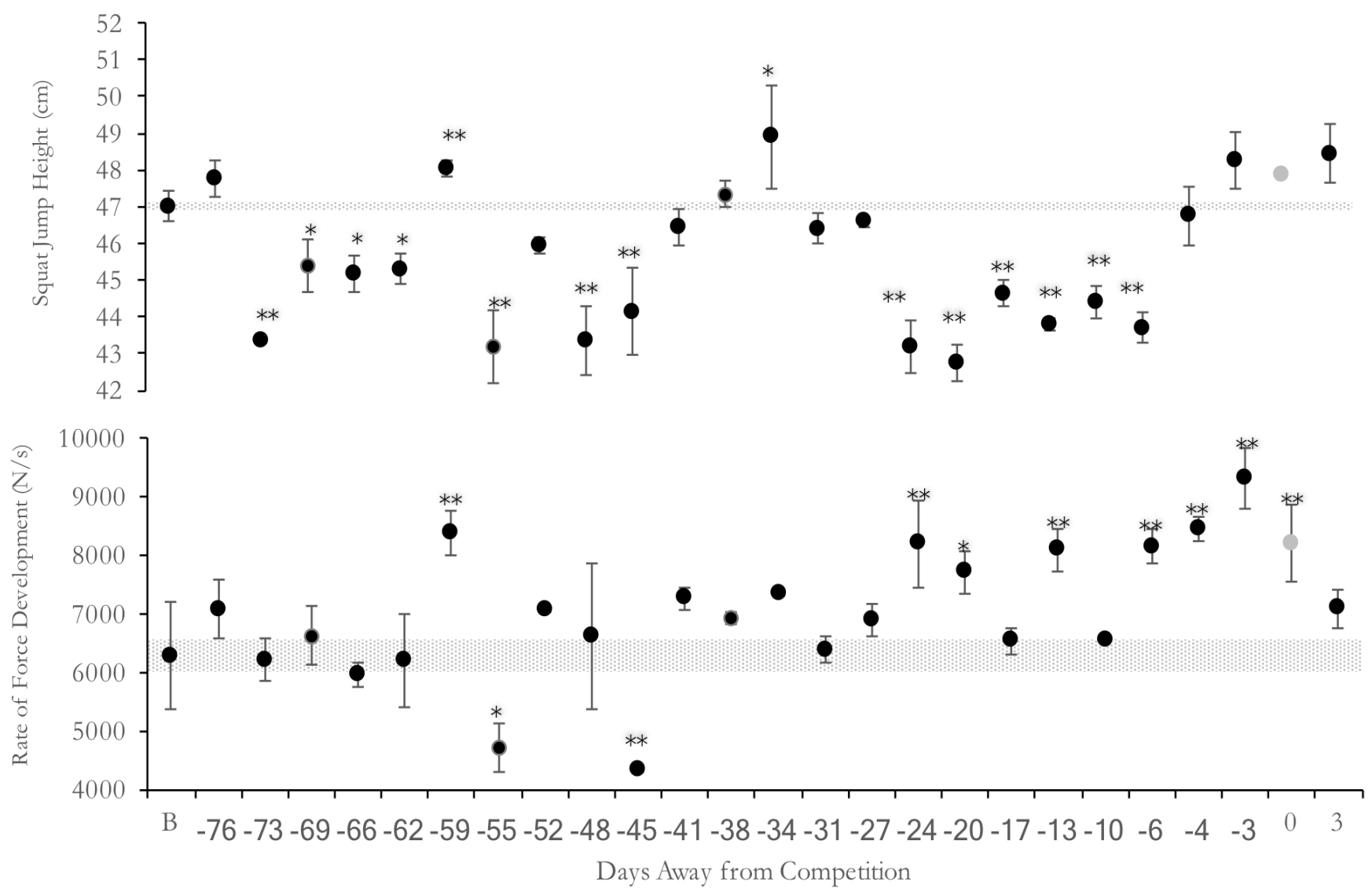

Figure 3.14 Jumping performance during Competition Preparation Phase (male). Note: *indicates $>95 \%$ (very likely), ** indicates $>99 \%$ (almost certainly). B: bassline testing sessions - average value during baseline measurements. Error bar is the standard deviation across baseline values. Gray region is the trivial or SWC. Gray marker above time point 0 is the day of competition.

\section{DISCUSSION}

To our knowledge, this was the first study to frequently monitor high-level weightlifters peaking and traveling to a national championship. The purpose of this study was to characterize when jump height was peaked for two high-level weightlifters relative to a major COMP and if any psychological or physiological changes could explain changes in jumping performance. The primary results of this investigation include: 1) COMP goals were met for the female athlete, but not for the male athlete, 2) muscle size was possibly not preserved following the taper for either athlete, 3) markers of training stress and inflammation exhibited inconsistent changes between 
athletes, 4) subjective recovery and stress scores improved leading to the COMP, and 5) SJH was peaked on COMP day with improvements above baseline beginning 3-4 days prior.

During the taper, the reduction in VL for both athletes was not only within the suggested reduction range for maximal strength (i.e., 30-70\%) (Pritchard et al., 2015) but the same amount for both athletes (48\%) and comparable to other tapering studies using weightlifters (Bazyler et al., 2018; Hornsby et al., 2017; Stone et al., 1996). The reductions observed for body mass and VL for each athlete may explain the downward trend observed in muscle size during the taper block. A similar relationship between body mass reduction ( $>3 \mathrm{kgs})$ and muscle size has been recently observed in a national level female weightlifter (Bazyler et al., 2018). However, further reductions below baseline in muscle size after the COMP was not expected despite the female athlete increasing body mass and the male athlete decreasing body mass leading to COMP day.

Our biochemical findings are not consistent with results from previous research on peaking and tapering for weightlifters relative to training VL (Bazyler et al., 2018; Busso et al., 1992; Fry et al., 2000; Häkkinen et al., 1987). Average training VL during the 17-week specific preparation phase prior to the competition phase (i.e., 11 weeks) for the male athlete was approximately $51,000 \mathrm{~kg}$ per block whereas the female averaged 27,000 kg per block. It has been suggested that female athletes are able to recover from training faster than male athletes possibly due to estrogen exerting a protective mechanism on muscle by limiting damage and accelerating the repair process (Enns \& Tiidus, 2010). Due to the overall higher training VL for the male athlete and a possible lower back injury, reductions greater than $48 \%$ may have been needed to negate $\mathrm{C}$ from increasing which possibly altered his performance. Negative residual training effects (i.e., 
fatigue) from the large surges of volume during overreach weeks could help explain why COMP performance was not met for the male athlete. For instance, accumulated fatigue and negative residual physiological effects have been shown to directly affect highly skilled motor tasks such as weightlifting technique and cognitive function which, in turn, may explain his performance (Blumert et al., 2007; Stone et al., 1993). It is also important to note that the male athlete had a slight back injury during training leading up to the major COMP that could have caused stress levels and serum concentrations to change in a negative manner. Häkkinen et al., (1987) reported that $\mathrm{T}: \mathrm{C}$ ratio increased following a taper in 13 elite level male weightlifters. Yet this was not consistent with our results. For example, the only trends for the female and male athletes observed was an increase in $\mathrm{T}$ the week of the final overreach ( 25 and 27 days, respectively, prior to COMP), increased $\mathrm{T}: \mathrm{C}$ ratio during week 2 (12 days and 14 days prior to COMP) of the taper, and increased CK after the COMP (5 days and 3 days, respectively). Although C fluctuated erratically prior to the COMP, steroid hormones were unpredictable throughout the study contrary to the current literature. It is possible that more frequent hormone monitoring would have produced responses consistent with previous literature, although this was not feasible in the present study.

Throughout the competition preparation period, for each athlete, levels of hsCRP and CK stayed below the clinical cutoff. Hecksteden et al. (2016) stated that hsCRP and CK are fatiguedependent markers that reflect muscle fiber damage from previous training sessions in elite athletes who strength train. Intensity was maintained during the taper to preserve neuromuscular adaptations and improve performance. Increases in intensity and maximal muscle tension required for performance tasks could partly explain why these markers did not return to baseline. 
It has also been suggested that $\mathrm{CK}$ is a more promising blood-borne indicator of fatigue for strength trained athletes because hsCRP returns to baseline relatively quickly (Hecksteden et al., 2016). Thus, confounding factors such as a residual training effects from the overreach and or COMP, a delayed physiological effect, traveling across multiple zones, adjustments in sleep patterns, food consumption, delayed onset of muscle soreness, etc. may explain the rise in inflammatory markers the week of and after COMP. As previously mentioned, the results from other studies observing biomarkers and cytokines in weightlifters have been inconsistent and seem to vary on a case-by-case basis (Bazyler et al., 2018). For instance, during week 3 of the taper, the male showed marked increases in $\mathrm{C}$ which may have accentuated the elevations in hsCRP and CK thereafter which are indicative of increased inflammation and persistent muscle soreness (Smith, 2000). Nevertheless, throughout the study, none of the steroid hormone markers were indicative of COMP results for the female athlete. However, it is plausible that the rise in the stress hormone $\mathrm{C}$ caused inflammatory responses to increase thereafter bringing insight into the COMP outcomes even though psychological and performance metrics showed that each athlete was in a peaked state of preparedness.

Although the biochemical and physiological measures of training strain were not consistent with previous results for either case, the psychological inventories for each athlete improved relative to the day of COMP. Every athlete possesses unique characteristics that are indicative of training-induced stress that may directly affect performance ability. For the female athlete, improved recovery scores were noticed 1 day prior to COMP whereas stress scores were only improved the day of COMP. The male athlete only showed improved recovery scores on COMP day whereas his stress scores were improving from 4 days prior up until the COMP day. These 
findings are comparable to studies that have shown the psychological inventories of high-level weightlifters can change dramatically at the COMP once the athlete perceives the importance of the COMP (Biddle, 1983; Mahoney, 1989). Perhaps, the SRSS scores reported on COMP day were most indicative of positive performance outcomes. Observing a female athlete's recovery subjectivity compared to a male athlete's recovery levels was expected regarding the differences in average VL discrepancies. For example, the average amount of work completed in a training block for the female compared to the male athlete was 1:2 (Refer to Figure 1 and Figure 2). Furthermore, elevated stress levels for the female athlete leading into the COMP should be expected considering previous findings that observed elite female weightlifters. This observation corresponds with previous findings by Mahoney (1989) indicating that in elite level female weightlifters stress can be a positive correlate to performance which is comparable to what was found in the present investigation.

Squat jump performance was considered to be peaked for both athletes the day after arrival and on each athlete's COMP day. Each athlete showed increased SJH and elevated RFD values the day after arrival, on COMP day, and after the COMP. For the male athlete, training VL seemed to directly influence fluctuations in SJH. During overreach weeks, SJH was suppressed and as the VL reduced SJH gradually increased. However, for the female athlete, the best jumping performances occurred during overreach and higher VL weeks throughout the study. These findings agree with literature suggesting that female athletes tend to show signs of being less acutely fatigued compared to male athletes after acute bouts of rigorous high-intensity training (Hunter, 2014). Thus, this may be indicative of an acute positive response female athletes acquire after an overreach where negative responses will be noticed once the residual 
fatigue effect accumulates days later. However, male athletes show negative effects almost immediately after an overreach period (Raeder et al., 2017) from accumulated fatigue which, in turn, requires a recovery period so positive performance can be expressed later on. These findings may give insight into how long high-level weightlifters are able to hold a peak relative to a major COMP. Considering these findings, $\mathrm{SJH}$ and the athlete's perceived stress and recovery state might provide a better indication of COMP preparedness relative to changes in training VL than serum blood markers in high-level weightlifters.

Conversely, although serum blood markers for stress, inflammation, and recovery have been previously related to manipulations in training VL these markers should be used and interpreted on a case-by-case basis (Bazyler et al., 2018). Even though there were small decreases in muscle size during the taper, this study was unable to observe single fiber adaptations or molecular signaling pathways which could explain and give insight into performance outcomes. Coupled with body mass reductions, the loss of muscle size could have been influenced by the amount of phosphagens, glycogen, water, etc. within the muscle tissue. Unattained COMP goals may have also been influenced by time differences from coast to coast resulting in inadequate sleep which has also been shown to influence complex motor task such as weightlifting movements, (Blumert et al., 2007) contrary to observed reductions in muscle size. Although COMP results were only met for the female athlete in this study, that does not mean that the peaking and tapering protocol was unsuccessful for the male athlete. Based on the jumping performance and SRSS results for each athlete, the tapering program was successful; however, there are other unmeasured factors that could confound competition performance (e.g., sleep quality and quantity, diet at the COMP site, weather, etc.). These findings provide insight into peaking for high-level weightlifters. 
Further investigations should observe molecular muscle changes during peaking phases as well as observe serum blood markers traveling to and on COMP day to better explain performance changes. Overall, these findings suggest that $\mathrm{SJH}$ coupled with recovery-stress scores might be used to assess a high-level weightlifter's preparedness leading up to a major COMP.

\section{CONCLUSION}

High-level athletes are unique because each athlete possesses characteristics that may be indicative of positive or negative performance changes leading up to an important COMP that may not have been previously observed. While the findings throughout the current literature are confounding, the high-level weightlifters in this investigation are distinct considering that both athletes showed psychological and physiological characteristics that were different and in some cases comparable leading up to the COMP. Yet, both athletes were considered to be in a peaked state on the day of COMP according to subjective recovery and stress, as well as jumping ability. Furthermore, this case series provides evidence for recommending the use of the SRSS and SJ as monitoring tools throughout a training year that uses successive overreaching and tapering periods to determine when weightlifters will express peak preparedness relative to the day of COMP. According to these findings and in these particular cases, high-level male and female weightlifters may expect to achieve and maintain peak preparedness 3-4 days prior to COMP following a 1-week overreach and 3-week taper.

\section{PRACTICAL APPLICATIONS}

This study provides insight into how high-level weightlifters prepared for a major national COMP and the underlying psychological and physiological processes explaining the 
performance outcomes. Considering possible reductions in muscle size within each athlete and the elevated inflammatory markers, sports scientists and coaches can use the SRSS and SJ testing to monitor changes in high-level weightlifter's preparedness leading up to an important COMP. It is also recommended that if traveling to an important COMP is involved, arriving several days prior to the event is important so that the window of peak preparedness can remain elevated relative to the day of COMP.

\section{ACKNOWLEDGMENT}

The authors would like to acknowledge the two graduate students who helped collect the data (Goodin and Perkins), the cooperation and involvement from the head weightlifting coach, and the athletes who participated in this study. Thank you all for your time and efforts.

\section{DISCLOSURE STATEMENT}

No potential conflict of interest reported by the authors.

\section{REFERENCES}

Abe, T., DeHoyos, D. V., Pollock, M. L., \& Garzarella, L. (2000). Time course for strength and muscle thickness changes following upper and lower body resistance training in men and women. European Journal of Applied Physiology, 81(3), 174-180.

https://doi.org/10.1007/s004210050027

Bazyler, C. D., Mizuguchi, S., Harrison, A. P., Sato, K., Kavanaugh, A. A., DeWeese, B. H., \& Stone, M. H. (2016). Changes in Muscle Architecture, Explosive Ability, and Track and 
Field Throwing Performance Throughout a Competitive Season and Following a Taper. Journal of Strength and Conditioning Research, Epub ahead of print.

Bazyler, C. D., Mizuguchi, S., Zourdos, M. C., Sato, K., Kavanaugh, A. A., DeWeese, B. H., ... Stone, M. H. (2018). Characteristics of a National Level Female Weightlifter Peaking for Competition: A Case Study. The Journal of Strength \& Conditioning Research, Publish Ahead of Print. https://doi.org/10.1519/JSC.0000000000002379

Biddle, S. J. H. (1983). Anxiety and Weightlifting: A Preliminary Analysis Of Anxiety Patterns and Coach Perceptions. Strength \& Conditioning Journal, 5(1), 40. Retrieved from https://journals.lww.com/nscascj/Citation/1983/02000/Anxiety and Weightlifting_A_Preliminary_Analysis.5.aspx

Blumert, P. A., Crum, A. J., Ernsting, M., Volek, J. S., Hollander, D. B., Haff, E. E., \& Haff, G. G. (2007). The acute effects of twenty-four hours of sleep loss on the performance of national-caliber male collegiate weightlifters. Journal of Strength and Conditioning Research, 21(4), 1146-1154. https://doi.org/10.1519/R-21606.1

Brechue, W. F., \& Abe, T. (2002). The role of FFM accumulation and skeletal muscle architecture in powerlifting performance. European Journal of Applied Physiology, 86(4), $327-336$.

Busso, T., Häkkinen, K., Pakarinen, A., Kauhanen, H., Komi, P. V., \& Lacour, J. R. (1992). Hormonal adaptations and modelled responses in elite weightlifters during 6 weeks of training. European Journal of Applied Physiology and Occupational Physiology, 64(4), 381386.

Carlock, J. M., Smith, S. L., Hartman, M. J., Morris, R. T., Ciroslan, D. A., Pierce, K. C., ... Stone, M. H. (2004). The relationship between vertical jump power estimates and 
weightlifting ability: a field-test approach. Journal of Strength and Conditioning Research, 18(3), 534-539. https://doi.org/10.1519/R-13213.1

Castro, M. J., McCann, D. J., Shaffrath, J. D., \& Adams, W. C. (1995). Peak torque per unit cross-sectional area differs between strength-trained and untrained young adults. Medicine and Science in Sports and Exercise, 27(3), 397-403.

Crewther, B. T., Heke, T., \& Keogh, J. W. L. (2011). The effects of training volume and competition on the salivary cortisol concentrations of Olympic weightlifters. Journal of Strength and Conditioning Research, 25(1), 10-15. https://doi.org/10.1519/JSC.0b013e3181fb47f5

Enns, D. L., \& Tiidus, P. M. (2010). The influence of estrogen on skeletal muscle: sex matters. Sports Medicine (Auckland, N.Z.), 40(1), 41-58. https://doi.org/10.2165/11319760$\underline{000000000-00000}$

Fry, A. C., Kraemer, W. J., Stone, M. H., Warren, B. J., Fleck, S. J., Kearney, J. T., \& Gordon, S. E. (1994). Endocrine responses to overreaching before and after 1 year of weightlifting. Canadian Journal of Applied Physiology = Revue Canadienne De Physiologie Appliquee, 19(4), 400-410.

Fry, A. C., Kraemer, W. J., Stone, M. H., Warren, B. J., Kearney, J. T., Maresh, C. M., ... Fleck, S. J. (1993). Endocrine and performance responses to high volume training and amino acid supplementation in elite junior weightlifters. International Journal of Sport Nutrition, 3(3), $306-322$.

Fry, Andrew C., Kraemer, W. J., Stone, M. H., Koziris, L. P., Thrush, J. T., \& Fleck, S. J. (2000). Relationships Between Serum Testosterone, Cortisol, and Weightlifting Performance. Journal of Strength and Conditioning Research, 14(3), 338-343. 
Fry, Andrew C., Schilling, B. K., Staron, R. S., Hagerman, F. C., Hikida, R. S., \& Thrush, J. T. (2003). Muscle fiber characteristics and performance correlates of male Olympic-style weightlifters. Journal of Strength and Conditioning Research, 17(4), 746-754.

Garhammer, J. (1980). Power production by Olympic weightlifters. Medicine and Science in Sports and Exercise, 12(1), 54-60.

Garhammer, J. (1993). A review of power output studies of olympic and powerlifting: methodology, performance prediction, and evaluation tests. Journal of Strength and Conditioning Research, 7(2), 76-89.

Garhammer, John, \& Gregor, R. (1992). Propulsion forces as a function of intensity for weightlifting and vertical jumping. Journal of Applied Sport Science Research, 6(3), 129134.

Gentles, J. A., Hornsby, W. G., Coniglio, C. L., Dotterweich, A. R., Miller, J. A., Stuart, C. A., \& Stone, M. H. (2017). Cell free DNA as a marker of training status in weightlifters. Biology of Sport, 34(3), 287-294. https://doi.org/10.5114/biolsport.2017.67855

Haff, G. G., Jackson, J. R., Kawamori, N., Carlock, J. M., Hartman, M. J., Kilgore, J. L., ... Stone, M. H. (2008). Force-time curve characteristics and hormonal alterations during an eleven-week training period in elite women weightlifters. Journal of Strength and Conditioning Research, 22(2), 433-446. https://doi.org/10.1519/JSC.0b013e31816191be

Häkkinen, K., Pakarinen, A., Alén, M., Kauhanen, H., \& Komi, P. V. (1987). Relationships between training volume, physical performance capacity, and serum hormone concentrations during prolonged training in elite weight lifters. International Journal of Sports Medicine, 8 Suppl 1, 61-65. 
Häkkinen, K., Pakarinen, A., Alen, M., Kauhanen, H., \& Komi, P. V. (1988). Neuromuscular and hormonal adaptations in athletes to strength training in two years. Journal of Applied Physiology (Bethesda, Md.: 1985), 65(6), 2406-2412.

https://doi.org/10.1152/jappl.1988.65.6.2406

Hartman, M. J., Clark, B., Bembens, D. A., Kilgore, J. L., \& Bemben, M. G. (2007).

Comparisons between twice-daily and once-daily training sessions in male weight lifters. International Journal of Sports Physiology and Performance, 2(2), 159-169.

Heath, B. H., \& Carter, J. E. (1967). A modified somatotype method. American Journal of Physical Anthropology, 27(1), 57-74.

Hecksteden, A., Skorski, S., Schwindling, S., Hammes, D., Pfeiffer, M., Kellmann, M., ... Meyer, T. (2016). Blood-Borne Markers of Fatigue in Competitive Athletes - Results from Simulated Training Camps. PLoS ONE, 11(2). https://doi.org/10.1371/journal.pone.0148810

Hopkins, W. G. (2000). Measures of reliability in sports medicine and science. Sports Medicine (Auckland, N.Z.), 30(1), 1-15.

Hopkins, W. G. (n.d.). A scaled of magnitudes for effect statistics [Internet]. Retrieved from http://www.sportsci.org/resource/stats/effectmag.html

Hopkins, W. G., Hawley, J. A., \& Burke, L. M. (1999). Design and analysis of research on sport performance enhancement. Medicine and Science in Sports and Exercise, 31(3), 472-485.

Hopkins, William G., Marshall, S. W., Batterham, A. M., \& Hanin, J. (2009). Progressive statistics for studies in sports medicine and exercise science. Medicine and Science in Sports and Exercise, 41(1), 3-13. https://doi.org/10.1249/MSS.0b013e31818cb278 
Hornsby, W. G., Gentles, J. A., MacDonald, C. J., Mizuguchi, S., Ramsey, M. W., \& Stone, M. H. (2017). Maximum strength, rate of force development, jump height, and peak power alterations in weightlifters across five months of training. Sports, 5(4), 78.

Howe, T. E., \& Oldham, J. A. (1996). The reliability of measuring quadriceps cross-sectional area with compound B ultrasound scanning. Physiotherapy Research International, 1(2), 112-126.

Hunter, S. K. (2014). Sex differences in human fatigability: mechanisms and insight to physiological responses. Acta Physiologica (Oxford, England), 210(4), 768-789. https://doi.org/10.1111/apha.12234

International Society for the Advancement of Kinathropometry. (2001). International Standards for Anthropometric Assessment. International Society for the Advancement of Kinathropometry.

Kellmann, M., Bertollo, M., Bosquet, L., Brink, M., Coutts, A. J., Duffield, R., .. Beckmann, J. (2018). Recovery and Performance in Sport: Consensus Statement. International Journal of Sports Physiology and Performance, 13(2), 240-245. https://doi.org/10.1123/ijspp.2017$\underline{0759}$

Kinugasa, T. (2013). The Application of Single-Case Research Designs to Study Elite Athletes' Conditioning: An Update. Journal of Applied Sport Psychology, 25(1), 157-166. https://doi.org/10.1080/10413200.2012.709578

Kölling, S., Hitzschke, B., Holst, T., Ferrauti, A., Meyer, T., Pfeiffer, M., \& Kellmann, M. (2015). Validity of the Acute Recovery and Stress Scale: Training Monitoring of the German Junior National Field Hockey Team. International Journal of Sports Science \& Coaching, 10(2-3), 529-542. https://doi.org/10.1260/1747-9541.10.2-3.529 
Kraemer, W. J., Fry, A. C., Warren, B. J., Stone, M. H., Fleck, S. J., Kearney, J. T., ... Triplett, N. T. (1992). Acute hormonal responses in elite junior weightlifters. International Journal of Sports Medicine, 13(2), 103-109. https://doi.org/10.1055/s-2007-1021240

Kraska, J. M., Ramsey, M. W., Haff, G. G., Fethke, N., Sands, W. A., Stone, M. E., \& Stone, M. H. (2009). Relationship Between Strength Characteristics and Unweighted and Weighted Vertical Jump Height. International Journal of Sports Physiology and Performance, 4(4), $461-473$.

Linthorne, N. P. (2001). Analysis of standing vertical jumps using a force platform. American Journal of Physics, 69(11), 1198-1204.

Mahoney, M. J. (1989). Psychological predictors of elite and non-elite performance in Olympic weightlifting. International Journal of Sport Psychology, 20(1), 1-12. Retrieved from https://www.cabdirect.org/cabdirect/abstract/19901874427

Mujika, I., \& Padilla, S. (2003). Scientific bases for precompetition tapering strategies. Medicine and Science in Sports and Exercise, 35(7), 1182-1187.

https://doi.org/10.1249/01.MSS.0000074448.73931.11

Nässi, A., Ferrauti, A., Meyer, T., Pfeiffer, M., \& Kellmann, M. (2017). Development of two short measures for recovery and stress in sport. European Journal of Sport Science, 17(7), 894-903. https://doi.org/10.1080/17461391.2017.1318180

Orvanová, E. (1990). Somatotypes of weight lifters. Journal of Sports Sciences, 8(2), 119-137. https://doi.org/10.1080/02640419008732139

Pelka, M., Schneider, P., \& Kellmann, M. (2017). Development of pre- and post-match morning recovery-stress states during in-season weeks in elite youth football. Science and Medicine in Football, O(0), 1-6. https://doi.org/10.1080/24733938.2017.1384560 
Pritchard, H., Keogh, J., Barnes, M., \& McGuigan, M. (2015). Effects and Mechanisms of Tapering in Maximizing Muscular Strength. Strength \& Conditioning Journal, 37(2). Retrieved from http://journals.lww.com/nscascj/Fulltext/2015/04000/Effects_and_Mechanisms_of_Tapering in_Maximizing.3.aspx

Raeder, C., Wiewelhove, T., Schneider, C., Döwelling, A., Kellmann, M., Meyer, T., ... Ferrauti, A. (2017). Effects of active recovery on muscle function following high-intensity training sessions in elite olympic weightlifters. Advances in Skeletal Muscle Function Assessment, $1(1), 3-12$.

Sale, D. G. (1988). Neural adaptation to resistance training. Medicine and Science in Sports and Exercise, 20(5 Suppl), S135-145.

Scanlon, T. C., Fragala, M. S., Stout, J. R., Emerson, N. S., Beyer, K. S., Oliveira, L. P., \& Hoffman, J. R. (2014). Muscle architecture and strength: adaptations to short-term resistance training in older adults. Muscle \& Nerve, 49(4), 584-592. https://doi.org/10.1002/mus.23969

Siart, B., Nimmerichter, A., Vidotto, C., \& Wallner, B. (2017). Status, Stress and Performance in Track and Field Athletes during the European Games in Baku (Azerbaijan). Scientific Reports, 7(1), 6076. https://doi.org/10.1038/s41598-017-06461-z

Smith, L. L. (2000). Cytokine hypothesis of overtraining: a physiological adaptation to excessive stress? Medicine and Science in Sports and Exercise, 32(2), 317-331.

Stone, M. H., Fry, A. C., Thrush, J. T., Fleck, S. J., Kraemer, W. J., Kearney, J. T., \& Marsit, J. (1993). Overtraining in weightlifters. Presented at the Weightlifting Symposium, Ancient Olympia, Greece. 
Stone, M. H., Josey, J., Hunter, G., Kearney, J. T., Fry, A. C., Kraemer, W. J., ... Haff, G. G. (1996). Different taper lengths: effects of weightlifting performance. In Overtraining and Overreaching in Sport Conference (p. 59). University of Memphis, TN.

Storey, A., \& Smith, H. K. (2012). Unique aspects of competitive weightlifting: performance, training and physiology. Sports Medicine (Auckland, N.Z.), 42(9), 769-790. https://doi.org/10.2165/11633000-000000000-00000

Thomas, L., \& Busso, T. (2005). A theoretical study of taper characteristics to optimize performance. Medicine and Science in Sports and Exercise, 37(9), 1615-1621.

Travis, S. K., Goodin, J. G., Suarez, D. G., \& Bazyler, C. D. (2017). Identifying a surrogate measure of weightlifting performance. Presented at the 12th Annual Coaching and Sport Science College, Johnson City, TN.

Viru, A. A., \& Viru, M. (2001). Biochemical monitoring of sport training. Champaign, IL : Human Kinetics. Retrieved from http://trove.nla.gov.au/version/44897223

Vizcaya, F. J., Viana, O., del Olmo, M. F., \& Acero, R. M. (2009). Could the deep squat jump predict weightlifting performance? Journal of Strength and Conditioning Research, 23(3), 729-734. https://doi.org/10.1519/JSC.0b013e3181a04dc3

Wu, C.-L., Hung, W., Wang, S.-Y., \& Chang, C.-K. (2008). Hormonal Responses in Heavy Training and Recovery Periods in an Elite Male Weightlifter. Journal of Sports Science \& Medicine, 7(4), 560-561. Retrieved from http://www.ncbi.nlm.nih.gov/pmc/articles/PMC3761924/ 


\section{CHAPTER 4}

\section{SUMMARY AND FUTURE INVESTIGATIONS}

The overall purpose of this thesis was to observe psychological, physiological and performance changes to determine when two high-level weightlifters were peaked for a major competition. This purpose was addressed by conducting a two-part case study series with one international level female and one national level male weightlifter. The primary results of this investigation include: 1) COMP goals were met for the female athlete, but not for the male athlete, 2) muscle size was not preserved following the taper for either case, 3) markers of training stress and inflammation exhibited inconsistent changes between both athletes, 4) subjective recovery and stress scores improved leading to the COMP, and 5) SJH was peaked on COMP day with improvements above baseline beginning 4 days and 3 days, respectively, prior to competing. Thus, our hypothesis that recovery-stress scores would improve, steroid hormone biomarkers would return to baseline, inflammatory markers would be reduced, muscle size would be preserved, and $\mathrm{SJH}$ would be peaked on the day of COMP following the taper was not fully supported.

Although overreaching periods may increase performance potential, a subsequent taper is needed to dissipate fatigue and express fitness prior to a competition (Busso et al., 1990). The taper is the most sensitive period of training when the training load should be reduced before a COMP in order to see a performance improvement (Mujika, 2009). This portion of the training regimen is aimed at expressing peak performance at the appropriate time for a major COMP. Tapering for sport has been documented extensively in the literature; however, the majority of 
the literature deals with endurance sport athletes and more recently team sport athletes (Murach \& Bagley, 2015).

Tapering essentially uses both art and science, but the exact nature of tapering is not fully understood. Currently, there is not an optimal tapering model used for weightlifters, and unfortunately, coaches use experience and intuition for athletes to peak on competition day (Thomas \& Busso, 2005). Throughout the literature review, tapering effects have shown consistently positive outcomes if programmed appropriately and accordingly for weightlifters (Bazyler et al., 2018; Hornsby et al., 2017; Stone et al., 1996). Although COMP goals were not met for the male case that does not suggest that the taper did not work. It is important to consider that even with the unmet COMP goals, the male weightlifter had a top 30 finish in the weight category with the largest group of lifters (i.e., 94kg). Psychological and physiological variables can aid in dictating an athlete's potential performance outcome letting the sport scientist and coach know when and if the athlete is ready for a competition. Sport scientist and coaches have been seeking the optimal tapering protocol for athletes at all levels for decades. Therefore, examining the time course of psychological, physiological, and performance changes of individual high-level weightlifters preparing for the same competition gives insight into identifying an optimal taper protocol for weightlifters.

With inconsistent serum steroid hormone levels for each athlete, the volatility for using such observations raises important concerns for reporting individual changes (i.e., SWC) instead of group means. Also, with the observed reductions in vastus lateralis MT and muscle CSA in this study, future research could expound upon our findings by investigating hypertrophic/atrophic 
intracellular signaling pathways. This would give insight into potential changes in protein synthesis, energy production pathways, etc. following structured peaking-tapering training blocks. Further, future research should compare the efficacy of different tapering models. For example, comparing exponential tapers with fast and slow decay, and step tapers may give more insight into establishing an optimal tapering protocol for weightlifters when preparing for major COMP. Nonetheless, this study provides novel insight into COMP preparation strategies for high-level weightlifters. This study contributes to filling the gap with underlying psychological and physiological processes that provides evidence for the performance outcomes of each case. Despite the physiological indicators of potential performance decrements in both cases, the SRSS and SJ testing provided valuable insight expounding upon the athlete's true COMP preparedness. Furthermore, this case series provides evidence for using the SRSS and SJ as monitoring tools throughout a training year that uses successive overreaching and tapering periods to determine when weightlifters will express peak preparedness relative to the day of COMP. 


\section{REFERENCES}

Abe, T., DeHoyos, D. V., Pollock, M. L., \& Garzarella, L. (2000). Time course for strength and muscle thickness changes following upper and lower body resistance training in men and women. European Journal of Applied Physiology, 81(3), 174-180.

https://doi.org/10.1007/s004210050027

Andersen, J. L., \& Aagaard, P. (2000). Myosin heavy chain IIX overshoot in human skeletal muscle. Muscle \& Nerve, 23(7), 1095-1104.

Aubry, A., Hausswirth, C., Louis, J., Coutts, A. J., \& LE Meur, Y. (2014). Functional overreaching: the key to peak performance during the taper? Medicine and Science in Sports and Exercise, 46(9), 1769-1777. https://doi.org/10.1249/MSS.0000000000000301

Banister, E. W., Carter, J. B., \& Zarkadas, P. C. (1999). Training theory and taper: validation in triathlon athletes. European Journal of Applied Physiology and Occupational Physiology, 79(2), 182-191. https://doi.org/10.1007/s004210050493

Barnett, A., Cerin, E., Reaburn, P., \& Hooper, S. (2010). The effects of training on performance and performance-related states in individual elite athletes: a dynamic approach. Journal of Sports Sciences, 28(10), 1117-1126. https://doi.org/10.1080/02640414.2010.497817

Bazyler, C. D., Mizuguchi, S., Zourdos, M. C., Sato, K., Kavanaugh, A. A., DeWeese, B. H., ... Stone, M. H. (2018). Characteristics of a National Level Female Weightlifter Peaking for Competition: A Case Study. The Journal of Strength \& Conditioning Research, Publish Ahead of Print. https://doi.org/10.1519/JSC.0000000000002379

Bazyler, C. D., Mizuguchi, S., Harrison, A. P., Sato, K., Kavanaugh, A. A., DeWeese, B. H., \& Stone, M. H. (2016). Changes in Muscle Architecture, Explosive Ability, and Track and 
Field Throwing Performance Throughout a Competitive Season and Following a Taper. Journal of Strength and Conditioning Research, Epub ahead of print.

Beckham, G., Mizuguchi, S., Carter, C., Sato, K., Ramsey, M., Lamont, H., ... Stone, M. (2013). Relationships of isometric mid-thigh pull variables to weightlifting performance. The Journal of Sports Medicine and Physical Fitness, 53(5), 573-581.

Biddle, S. J. H. (1983). Anxiety and Weightlifting: A Preliminary Analysis Of Anxiety Patterns and Coach Perceptions. Strength \& Conditioning Journal, 5(1), 40. Retrieved from https://journals.lww.com/nscascj/Citation/1983/02000/Anxiety and Weightlifting A Preliminary Analysis.5.aspx

Blumert, P. A., Crum, A. J., Ernsting, M., Volek, J. S., Hollander, D. B., Haff, E. E., \& Haff, G. G. (2007). The acute effects of twenty-four hours of sleep loss on the performance of national-caliber male collegiate weightlifters. Journal of Strength and Conditioning Research, 21(4), 1146-1154. https://doi.org/10.1519/R-21606.1

Bosquet, L., Montpetit, J., Arvisais, D., \& Mujika, I. (2007). Effect of Tapering on Performance: A Meta-Analysis, 39(8), 1358-1365.

Brännström, A., Rova, A., \& Yu, J.-G. (2013). Effects and Mechanisms of Tapering in Maximizing Muscular Power. International Journal of Human Movement and Sports Sciences, 1(1), 18-23. https://doi.org/10.13189/saj.2013.010103

Brechue, W. F., \& Abe, T. (2002). The role of FFM accumulation and skeletal muscle architecture in powerlifting performance. European Journal of Applied Physiology, 86(4), $327-336$.

Busso, T., Häkkinen, K., Pakarinen, A., Carasso, C., Lacour, J. R., Komi, P. V., \& Kauhanen, H. (1990). A systems model of training responses and its relationship to hormonal responses in 
elite weight-lifters. European Journal of Applied Physiology and Occupational Physiology, 61(1-2), 48-54. https://doi.org/10.1007/BF00236693

Busso, T., Häkkinen, K., Pakarinen, A., Kauhanen, H., Komi, P. V., \& Lacour, J. R. (1992). Hormonal adaptations and modelled responses in elite weightlifters during 6 weeks of training. European Journal of Applied Physiology and Occupational Physiology, 64(4), 381386.

Busso, Thierry, Candau, R., \& Lacour, J.-R. (1994). Fatigue and fitness modelled from the effects of training on performance. European Journal of Applied Physiology and Occupational Physiology, 69(1), 50-54. https://doi.org/10.1007/BF00867927

Caporale, A. (2017, January). Influence of the Menstrual Cycle and its Phases on the Performance of Olympic Weightlifting Athletes. The Official Journal of the European Weightlifting Federation, 3(6), 30-43.

Cardinale, M., \& Stone, M. H. (2006). Is testosterone influencing explosive performance? Journal of Strength and Conditioning Research, 20(1), 103-107. https://doi.org/10.1519/R$\underline{16864.1}$

Carlock, J. M., Smith, S. L., Hartman, M. J., Morris, R. T., Ciroslan, D. A., Pierce, K. C., ... Stone, M. H. (2004). The relationship between vertical jump power estimates and weightlifting ability: a field-test approach. Journal of Strength and Conditioning Research, 18(3), 534-539. https://doi.org/10.1519/R-13213.1

Castro, M. J., McCann, D. J., Shaffrath, J. D., \& Adams, W. C. (1995). Peak torque per unit cross-sectional area differs between strength-trained and untrained young adults. Medicine and Science in Sports and Exercise, 27(3), 397-403. 
Chiu, L. Z. F., \& Barnes, J. L. (2003). The Fitness-Fatigue Model Revisited: Implications for Planning Short- and Long-Term Training. Strength \& Conditioning Journal, 25(6), 42. Retrieved from https://journals.lww.com/nscascj/Citation/2003/12000/The Fitness_Fatigue Model_Revisited_Implications.7.aspx

Clarkson, P. M., Kroll, W., \& McBride, T. C. (1980). Maximal isometric strength and fiber type composition in power and endurance athletes. European Journal of Applied Physiology and Occupational Physiology, 44(1), 35-42. https://doi.org/10.1007/BF00421761

Cook, C. J., Crewther, B. T., \& Smith, A. A. (2012). Comparison of baseline free testosterone and cortisol concentrations between elite and non-elite female athletes. American Journal of Human Biology: The Official Journal of the Human Biology Council, 24(6), 856-858. https://doi.org/10.1002/ajhb.22302

Coutts, A., Reaburn, P., Piva, T. J., \& Murphy, A. (2007). Changes in selected biochemical, muscular strength, power, and endurance measures during deliberate overreaching and tapering in rugby league players. International Journal of Sports Medicine, 28(2), 116-124. https://doi.org/10.1055/s-2006-924145

Crewther, B. T., Obmiński, Z., \& Cook, C. J. (2018). Serum cortisol as a moderator of the relationship between serum testosterone and olympic weightlifting performance in real and simulated competitions. Biology of Sport, 35(3), 215-221.

DeWeese, B. H., Hornsby, G., Stone, M., \& Stone, M. H. (2015a). The training process: Planning for strength-power training in track and field. Part 1: Theoretical aspects. Journal of Sport and Health Science, 4(4), 308-317. https://doi.org/10.1016/j.jshs.2015.07.003

DeWeese, B. H., Hornsby, G., Stone, M., \& Stone, M. H. (2015b). The training process: Planning for strength-power training in track and field. Part 2: Practical and applied aspects. 
Journal of Sport and Health Science, 4(4), 318-324.

https://doi.org/10.1016/j.jshs.2015.07.002

Di Naso, J. J., Pritschet, B. L., Emmett, J. D., Owen, J. D., Willardson, J. M., Beck, T. W., ,.. Fontana, F. E. (2012). Comparing thigh muscle cross-sectional area and squat strength among national class Olympic weightlifters, power lifters, and bodybuilders. International SportMed Journal, 13(2), 48-57.

Drechsler, A. J. (1998). The weightlifting encyclopedia: a guide to world class performance. Presented at the A is A Communications, Whitestone (NY).

Durguerian, A., Bougard, C., Drogou, C., Sauvet, F., Chennaoui, M., \& Filaire, E. (2016). Weight Loss, Performance and Psychological Related States in High-level Weightlifters. International Journal of Sports Medicine, 37(3), 230-238. https://doi.org/10.1055/s-0035$\underline{1555852}$

Fair, J. D. (1988). Olympic weightlifting and the introduction of steroids: a statistical analysis of world championship results, 1948-72. The International Journal of the History of Sport, 5(1), 96-114. https://doi.org/10.1080/09523368808713646

Franke, W. W., \& Berendonk, B. (1997). Hormonal doping and androgenization of athletes: a secret program of the German Democratic Republic government. Clinical Chemistry, 43(7), 1262-1279. Retrieved from http://clinchem.aaccjnls.org/content/43/7/1262

Fry, A. C., \& Kraemer, W. J. (1997). Resistance exercise overtraining and overreaching. Neuroendocrine responses. Sports Medicine (Auckland, N.Z.), 23(2), 106-129.

Fry, A. C., Kraemer, W. J., Stone, M. H., Warren, B. J., Fleck, S. J., Kearney, J. T., \& Gordon, S. E. (1994). Endocrine responses to overreaching before and after 1 year of weightlifting. 
Canadian Journal of Applied Physiology = Revue Canadienne De Physiologie Appliquee, 19(4), 400-410.

Fry, Andrew C. (2004). The role of resistance exercise intensity on muscle fibre adaptations. Sports Medicine (Auckland, N.Z.), 34(10), 663-679.

Fry, Andrew C., Ciroslan, D., Fry, M. D., LeRoux, C. D., Schilling, B. K., \& Chiu, L. Z. F. (2006). Anthropometric and performance variables discriminating elite American junior men weightlifters. Journal of Strength and Conditioning Research, 20(4), 861-866. https://doi.org/10.1519/R-18355.1

Fry, Andrew C., Kraemer, W. J., Stone, M. H., Koziris, L. P., Thrush, J. T., \& Fleck, S. J. (2000). Relationships Between Serum Testosterone, Cortisol, and Weightlifting Performance. Journal of Strength and Conditioning Research, 14(3), 338-343.

Fry, Andrew C., Schilling, B. K., Staron, R. S., Hagerman, F. C., Hikida, R. S., \& Thrush, J. T. (2003). Muscle fiber characteristics and performance correlates of male Olympic-style weightlifters. Journal of Strength and Conditioning Research, 17(4), 746-754.

Fullagar, H. H. K., McCunn, R., \& Murray, A. (2017). An Updated Review of the Applied Physiology of American Collegiate Football: The Physical Demands, Strength/Conditioning, Nutritional Considerations and Injury Characteristics of America's Favourite Game. International Journal of Sports Physiology and Performance, 1-27. https://doi.org/10.1123/ijspp.2016-0783

Funato, K., Kanehisa, H., \& Fukunaga, T. (2000). Differences in muscle cross-sectional area and strength between elite senior and college Olympic weight lifters. The Journal of Sports Medicine and Physical Fitness, 40(4), 312-318. 
García-Pallarés, J., Sánchez-Medina, L., Pérez, C. E., Izquierdo-Gabarren, M., \& Izquierdo, M. (2010). Physiological effects of tapering and detraining in world-class kayakers. Medicine and Science in Sports and Exercise, 42(6), 1209-1214.

https://doi.org/10.1249/MSS.0b013e3181c9228c

Garhammer, J. (1979). Longitudinal analysis of highly skilled Olympic weightlifters. Science in Weightlifting, 79-88.

Garhammer, J. (1980). Power production by Olympic weightlifters. Medicine and Science in Sports and Exercise, 12(1), 54-60.

Garhammer, J. (1985). Biomechanical profiles of Olympic weightlifters. International Journal of Sport Biomechanics, 1, 122-130.

Garhammer, J. (1993). A review of power output studies of olympic and powerlifting: methodology, performance prediction, and evaluation tests. Journal of Strength and Conditioning Research, 7(2), 76-89.

Garhammer, John, \& Gregor, R. (1992). Propulsion forces as a function of intensity for weightlifting and vertical jumping. Journal of Applied Sport Science Research, 6(3), 129134.

Garhammer, John, \& Takano, B. (2003). Training for Weightlifting. In P. V. Komi (Ed.), Strength and Power in Sport (pp. 502-515). Blackwell Science Ltd. https://doi.org/10.1002/9780470757215.ch25

Gentles, J. A., Hornsby, W. G., Coniglio, C. L., Dotterweich, A. R., Miller, J. A., Stuart, C. A., \& Stone, M. H. (2017). Cell free DNA as a marker of training status in weightlifters. Biology of Sport, 34(3), 287-294. https://doi.org/10.5114/biolsport.2017.67855 
Gleeson, M., McDonald, W. A., Pyne, D. B., Clancy, R. L., Cripps, A. W., Francis, J. L., \& Fricker, P. A. (2000). Immune status and respiratory illness for elite swimmers during a 12week training cycle. International Journal of Sports Medicine, 21(4), 302-307. https://doi.org/10.1055/s-2000-313

Gollnick, P. D., Armstrong, R. B., Saubert, C. W., Piehl, K., \& Saltin, B. (1972). Enzyme activity and fiber composition in skeletal muscle of untrained and trained men. Journal of Applied Physiology, 33(3), 312-319. https://doi.org/10.1152/jappl.1972.33.3.312

González-Badillo, J. J., Izquierdo, M., \& Gorostiaga, E. M. (2006). Moderate volume of high relative training intensity produces greater strength gains compared with low and high volumes in competitive weightlifters. Journal of Strength and Conditioning Research, 20(1), 73-81. https://doi.org/10.1519/R-16284.1

Grgic, J., \& Mikulic, P. (2017). Tapering Practices of Croatian Open-Class Powerlifting Champions. The Journal of Strength \& Conditioning Research, 31(9), 2371. https://doi.org/10.1519/JSC.0000000000001699

Haff, G. G., Carlock, J. M., Hartman, M. J., Kilgore, J. L., Kawamori, N., Jackson, J. R., ... Stone, M. H. (2005). Force-time curve characteristics of dynamic and isometric muscle actions of elite women olympic weightlifters. The Journal of Strength \& Conditioning Research, 19(4), 741. Retrieved from http://journals.lww.com/nscajscr/Abstract/2005/11000/FORCE TIME CURVE CHARACTERISTICS OF DYNAMI C AND.4.aspx

Haff, G. G., Jackson, J. R., Kawamori, N., Carlock, J. M., Hartman, M. J., Kilgore, J. L., ... Stone, M. H. (2008). Force-time curve characteristics and hormonal alterations during an 
eleven-week training period in elite women weightlifters. Journal of Strength and Conditioning Research, 22(2), 433-446. https://doi.org/10.1519/JSC.0b013e31816191be

Haff, G. G., Stone, M., O’Bryant, H. S., Harman, E., Dinan, C., Johnson, R., \& Han, K.-H. (1997). Force-Time Dependent Characteristics of Dynamic and Isometric Muscle Actions. The Journal of Strength \& Conditioning Research, 11(4), 269. Retrieved from https://journals.1ww.com/nscajscr/abstract/1997/11000/force time dependent characteristics of dynamic.14.aspx

Häkkinen, K., Alén, M., \& Komi, P. V. (1984). Neuromuscular, anaerobic, and aerobic performance characteristics of elite power athletes. European Journal of Applied Physiology and Occupational Physiology, 53(2), 97-105.

Häkkinen, K., \& Kallinen, M. (1994). Distribution of strength training volume into one or two daily sessions and neuromuscular adaptations in female athletes. Electromyography and Clinical Neurophysiology, 34(2), 117-124.

Häkkinen, K., Kallinen, M., Komi, P. V., \& Kauhanen, H. (1991). Neuromuscular adaptations during short-term "normal" and reduced training periods in strength athletes. Electromyography and Clinical Neurophysiology, 31(1), 35-42.

Häkkinen, K., \& Komi, P. V. (1983). Electromyographic changes during strength training and detraining. Medicine and Science in Sports and Exercise, 15(6), 455-460.

Häkkinen, K., Komi, P. V., \& Kauhanen, H. (1986). Electromyographic and force production characteristics of leg extensor muscles of elite weight lifters during isometric, concentric, and various stretch-shortening cycle exercises. International Journal of Sports Medicine, 7(3), 144-151. https://doi.org/10.1055/s-2008-1025752 
Häkkinen, K., Pakarinen, A., Alén, M., Kauhanen, H., \& Komi, P. V. (1987). Relationships between training volume, physical performance capacity, and serum hormone concentrations during prolonged training in elite weight lifters. International Journal of Sports Medicine, 8 Suppl 1, 61-65.

Häkkinen, K., Pakarinen, A., Alen, M., Kauhanen, H., \& Komi, P. V. (1988). Neuromuscular and hormonal adaptations in athletes to strength training in two years. Journal of Applied Physiology (Bethesda, Md.: 1985), 65(6), 2406-2412.

https://doi.org/10.1152/jappl.1988.65.6.2406

Häkkinen, K., Pakarinen, A., Alén, M., \& Komi, P. V. (1985). Serum hormones during prolonged training of neuromuscular performance. European Journal of Applied Physiology and Occupational Physiology, 53(4), 287-293.

Halson, S. L. (2014). Monitoring Training Load to Understand Fatigue in Athletes. Sports Medicine (Auckland, N.Z.), 44(Suppl 2), 139-147. https://doi.org/10.1007/s40279-014-0253$\underline{\mathrm{Z}}$

Halson, S. L., Bridge, M. W., Meeusen, R., Busschaert, B., Gleeson, M., Jones, D. A., \& Jeukendrup, A. E. (2002). Time course of performance changes and fatigue markers during intensified training in trained cyclists. Journal of Applied Physiology (Bethesda, Md.: 1985), 93(3), 947-956. https://doi.org/10.1152/japplphysiol.01164.2001

Hamilton, D. (2012). The impact of monitoring strategies on a team sport through an olympiad: physical development, taper \& recovery. Lecture presented at the UKSCA Annual Conference, Royal Holloway Univeristy, Egham, London. 
Hartman, M. J., Clark, B., Bembens, D. A., Kilgore, J. L., \& Bemben, M. G. (2007).

Comparisons between twice-daily and once-daily training sessions in male weight lifters.

International Journal of Sports Physiology and Performance, 2(2), 159-169.

Hecksteden, A., Skorski, S., Schwindling, S., Hammes, D., Pfeiffer, M., Kellmann, M., ...

Meyer, T. (2016). Blood-Borne Markers of Fatigue in Competitive Athletes - Results from

Simulated Training Camps. PLoS ONE, 11(2). https://doi.org/10.1371/journal.pone.0148810

Holloway, J. B., \& Baechle, T. R. (1990). Strength Training for Female Athletes. Sports

Medicine, 9(4), 216-228. https://doi.org/10.2165/00007256-199009040-00003

Hooper, D. R., Kraemer, W. J., Focht, B. C., Volek, J. S., DuPont, W. H., Caldwell, L. K., \&

Maresh, C. M. (2017). Endocrinological Roles for Testosterone in Resistance Exercise

Responses and Adaptations. Sports Medicine (Auckland, N.Z.), 47(9), 1709-1720.

https://doi.org/10.1007/s40279-017-0698-y

Hopkins, W. G. (2000). Measures of reliability in sports medicine and science. Sports Medicine (Auckland, N.Z.), 30(1), 1-15.

Hopkins, W. G. (n.d.). A scaled of magnitudes for effect statistics [Internet]. Retrieved from http://www.sportsci.org/resource/stats/effectmag.html

Hopkins, W. G., Hawley, J. A., \& Burke, L. M. (1999). Design and analysis of research on sport performance enhancement. Medicine and Science in Sports and Exercise, 31(3), 472-485.

Hopkins, William G., Marshall, S. W., Batterham, A. M., \& Hanin, J. (2009). Progressive statistics for studies in sports medicine and exercise science. Medicine and Science in Sports and Exercise, 41(1), 3-13. https://doi.org/10.1249/MSS.0b013e31818cb278

Hornsby, W. (2013). A Systematic Approach to the Monitoring of Competitive Weightlifters. Electronic Theses and Dissertations. Retrieved from https://dc.etsu.edu/etd/1189 
Hornsby, W. G., Gentles, J. A., MacDonald, C. J., Mizuguchi, S., Ramsey, M. W., \& Stone, M. H. (2017). Maximum strength, rate of force development, jump height, and peak power alterations in weightlifters across five months of training. Sports, 5(4), 78.

Houmard, J. A. (1991). Impact of reduced training on performance in endurance athletes. Sports Medicine (Auckland, N.Z.), 12(6), 380-393.

Howe, T. E., \& Oldham, J. A. (1996). The reliability of measuring quadriceps cross-sectional area with compound B ultrasound scanning. Physiotherapy Research International, 1(2), $112-126$.

Hunter, S. K. (2014). Sex differences in human fatigability: mechanisms and insight to physiological responses. Acta Physiologica (Oxford, England), 210(4), 768-789. https://doi.org/10.1111/apha.12234

Ikai, M., \& Fukunaga, T. (1968). Calculation of muscle strength per unit cross-sectional area of human muscle by means of ultrasonic measurement. Internationale Zeitschrift Fur Angewandte Physiologie, Einschliesslich Arbeitsphysiologie, 26(1), 26-32.

International Society for the Advancement of Kinathropometry. (2001). International Standards for Anthropometric Assessment. International Society for the Advancement of Kinathropometry.

Izquierdo, M., Ibañez, J., Calbet, J. A. L., Navarro-Amezqueta, I., González-Izal, M., Idoate, F., ... Gorostiaga, E. M. (2009). Cytokine and hormone responses to resistance training. European Journal of Applied Physiology, 107(4), 397-409. https://doi.org/10.1007/s00421$\underline{009-1139-\mathrm{X}}$

Izquierdo, M., Ibañez, J., González-Badillo, J. J., Ratamess, N. A., Kraemer, W. J., Häkkinen, K., ... Gorostiaga, E. M. (2007). Detraining and tapering effects on hormonal responses and 
strength performance. Journal of Strength and Conditioning Research, 21(3), 768-775. https://doi.org/10.1519/R-21136.1

Izquierdo, M., Ibáñez, J., Häkkinen, K., Kraemer, W. J., Ruesta, M., \& Gorostiaga, E. M. (2004). Maximal strength and power, muscle mass, endurance and serum hormones in weightlifters and road cyclists. Journal of Sports Sciences, 22(5), 465-478. https://doi.org/10.1080/02640410410001675342

Katch, V. L., Katch, F. I., Moffatt, R., \& Gittleson, M. (1980). Muscular development and lean body weight in body builders and weight lifters. Medicine and Science in Sports and Exercise, 12(5), 340-344.

Kauhanen, H., Garhammer, J., \& Häkkinen, K. (2000). Relationship between power output, body size and snatch performance in elite weightlifters. In 5th Annual Congress of the European College of Sport Sciences. Jyvaskala, Finland.

Kawakami, Y., Abe, T., \& Fukunaga, T. (1993). Muscle-fiber pennation angles are greater in hypertrophied than in normal muscles. Journal of Applied Physiology (Bethesda, Md.: 1985), 74(6), 2740-2744. https://doi.org/10.1152/jappl.1993.74.6.2740

Kellmann, M., \& Günther, K. D. (2000). Changes in stress and recovery in elite rowers during preparation for the Olympic Games. Medicine and Science in Sports and Exercise, 32(3), 676-683.

Kellmann, Michael, Bertollo, M., Bosquet, L., Brink, M., Coutts, A. J., Duffield, R., ... Beckmann, J. (2018). Recovery and Performance in Sport: Consensus Statement. International Journal of Sports Physiology and Performance, 13(2), 240-245. https://doi.org/10.1123/ijspp.2017-0759 
Kenney, W. L., Wilmore, J., \& Costill, D. L. (2015). Physiology of Sport and Exercise (6th ed.). Chicago, IL: Human Kinetics.

Kinugasa, T. (2013). The Application of Single-Case Research Designs to Study Elite Athletes' Conditioning: An Update. Journal of Applied Sport Psychology, 25(1), 157-166. https://doi.org/10.1080/10413200.2012.709578

Kishali, N. F., Imamoglu, O., Katkat, D., Atan, T., \& Akyol, P. (2006). Effects of menstrual cycle on sports performance. The International Journal of Neuroscience, 116(12), 15491563. https://doi.org/10.1080/00207450600675217

Kölling, S., Hitzschke, B., Holst, T., Ferrauti, A., Meyer, T., Pfeiffer, M., \& Kellmann, M. (2015). Validity of the Acute Recovery and Stress Scale: Training Monitoring of the German Junior National Field Hockey Team. International Journal of Sports Science \& Coaching, 10(2-3), 529-542. https://doi.org/10.1260/1747-9541.10.2-3.529

Kraemer, W. J., Fry, A. C., Warren, B. J., Stone, M. H., Fleck, S. J., Kearney, J. T., ... Triplett, N. T. (1992). Acute hormonal responses in elite junior weightlifters. International Journal of Sports Medicine, 13(2), 103-109. https://doi.org/10.1055/s-2007-1021240

Kraska, J. M., Ramsey, M. W., Haff, G. G., Fethke, N., Sands, W. A., Stone, M. E., \& Stone, M. H. (2009). Relationship Between Strength Characteristics and Unweighted and Weighted Vertical Jump Height. International Journal of Sports Physiology and Performance, 4(4), $461-473$.

Lewis, D. A., Kamon, E., \& Hodgson, J. L. (1986). Physiological differences between genders. Implications for sports conditioning. Sports Medicine (Auckland, N.Z.), 3(5), 357-369.

Linthorne, N. P. (2001). Analysis of standing vertical jumps using a force platform. American Journal of Physics, 69(11), 1198-1204. 
Loenneke, J. P., Wilson, G. J., \& Wilson, J. M. (2010). A mechanistic approach to blood flow occlusion. International Journal of Sports Medicine, 31(1), 1-4. https://doi.org/10.1055/s$\underline{0029-1239499}$

Lorenz, D. S., Reiman, M. P., Lehecka, B. J., \& Naylor, A. (2013). What Performance Characteristics Determine Elite Versus Nonelite Athletes in the Same Sport? Sports Health, 5(6), 542-547. https://doi.org/10.1177/1941738113479763

Lüthi, J. M., Howald, H., Claassen, H., Rösler, K., Vock, P., \& Hoppeler, H. (1986). Structural changes in skeletal muscle tissue with heavy-resistance exercise. International Journal of Sports Medicine, 7(3), 123-127. https://doi.org/10.1055/s-2008-1025748

Maffiuletti, N. A., Aagaard, P., Blazevich, A. J., Folland, J., Tillin, N., \& Duchateau, J. (2016). Rate of force development: physiological and methodological considerations. European Journal of Applied Physiology, 116(6), 1091-1116. https://doi.org/10.1007/s00421-0163346-6

Mahoney, M. J. (1989). Psychological predictors of elite and non-elite performance in Olympic weightlifting. International Journal of Sport Psychology, 20(1), 1-12. Retrieved from https://www.cabdirect.org/cabdirect/abstract/19901874427

Malisoux, L., Francaux, M., \& Theisen, D. (2007). What do single-fiber studies tell us about exercise training? Medicine and Science in Sports and Exercise, 39(7), 1051-1060. https://doi.org/10.1249/mss.0b13e318057aeb

Maynar, M., Timon, R., González, A., Olcina, G., Toribio, F., Maynar, J. I., \& Caballero, M. J. (2010). SHBG, plasma, and urinary androgens in weight lifters after a strength training. Journal of Physiology and Biochemistry, 66(2), 137-142. https://doi.org/10.1007/s13105$\underline{010-0018-6}$ 
Mcbride, J. M., Triplett-Mcbride, T., Davie, A., \& Newton, R. U. (1999). A Comparison of Strength and Power Characteristics Between Power Lifters, Olympic Lifters, and Sprinters. The Journal of Strength \& Conditioning Research, 13(1), 58. Retrieved from http://journals.lww.com/nscajscr/Abstract/1999/02000/A_Comparison_of_Strength_and_Power_Characteristics.11.aspx

McGuigan, M. R., \& Kane, M. K. (2004). Reliability of performance of elite Olympic weightlifters. Journal of Strength and Conditioning Research, 18(3), 650-653. https://doi.org/10.1519/12312.1

McMahon, J. J., Turner, A., \& Comfort, P. (2015). Relationships between lower body muscle structure and maximal power clean performance. Journal of Trainology, 4(2), 32-36. https://doi.org/10.17338/trainology.4.2 32

Meeusen, R., Duclos, M., Foster, C., Fry, A., Gleeson, M., Nieman, D., ... American College of Sports Medicine. (2013). Prevention, diagnosis, and treatment of the overtraining syndrome: joint consensus statement of the European College of Sport Science and the American College of Sports Medicine. Medicine and Science in Sports and Exercise, 45(1), 186-205. https://doi.org/10.1249/MSS.0b013e318279a10a

Morgan, W. P., Brown, D. R., Raglin, J. S., O’Connor, P. J., \& Ellickson, K. A. (1987). Psychological monitoring of overtraining and staleness. British Journal of Sports Medicine, 21(3), 107-114. Retrieved from http://www.ncbi.nlm.nih.gov/pmc/articles/PMC1478455/ Mujika, I. (2010). Intense training: the key to optimal performance before and during the taper. Scandinavian Journal of Medicine \& Science in Sports, 20 Suppl 2, 24-31. https://doi.org/10.1111/j.1600-0838.2010.01189.x 
Mujika, I., Chatard, J. C., \& Geyssant, A. (1996). Effects of training and taper on blood leucocyte populations in competitive swimmers: relationships with cortisol and performance. International Journal of Sports Medicine, 17(3), 213-217. https://doi.org/10.1055/s-2007$\underline{972834}$

Mujika, I., Padilla, S., \& Pyne, D. (2002). Swimming performance changes during the final 3 weeks of training leading to the Sydney 2000 Olympic Games. International Journal of Sports Medicine, 23(8), 582-587. https://doi.org/10.1055/s-2002-35526

Mujika, I. (2009). Tapering and Peaking for Optimal Performance (Illustrated). Champaign, IL: Human Kinetic.

Mujika, I, Goya, A., Padilla, S., Grijalba, A., Gorostiaga, E., \& Ibañez, J. (2000). Physiological Responses to a 6-d Taper in Middle-Distance Runners: Influence of Training Intensity and Volume. Medicine \& Science in Sports and Exercise, 32(2), 511-517.

Mujika, I, \& Padilla, S. (2000). Detraining: Loss of Training-Induced Physiological and Performance Adaptations. Part I. Sports Medicine, 30(2), 79-87. https://doi.org/10.2165/00007256-200030020-00002

Mujika, I., \& Padilla, S. (2003). Scientific bases for precompetition tapering strategies. Medicine and Science in Sports and Exercise, 35(7), 1182-1187. https://doi.org/10.1249/01.MSS.0000074448.73931.11

Mujika, Iñigo, Padilla, S., Pyne, D., \& Busso, T. (2004). Physiological changes associated with the pre-event taper in athletes. Sports Medicine (Auckland, N.Z.), 34(13), 891-927.

Murach, K. A., \& Bagley, J. R. (2015). Less Is More: The Physiological Basis for Tapering in Endurance, Strength, and Power Athletes. Sports, 3(3), 209-218. 
Murach, K., Raue, U., Wilkerson, B., Minchev, K., Jemiolo, B., Bagley, J., ... Trappe, S. (2014). Single muscle fiber gene expression with run taper. PloS One, 9(9), e108547. https://doi.org/10.1371/journal.pone.0108547

Myers, V. R., McKillop, A. L., Fraser, S. J., Abel, J. M., \& Wells, G. D. (2017). Physiological and psychological adaptations during taper in competitive swimmers. International Journal of Sports Science \& Coaching, 12(4), 481-494. https://doi.org/10.1177/1747954117717878

Nässi, A., Ferrauti, A., Meyer, T., Pfeiffer, M., \& Kellmann, M. (2017). Development of two short measures for recovery and stress in sport. European Journal of Sport Science, 17(7), 894-903. https://doi.org/10.1080/17461391.2017.1318180

Neary, J. P., Martin, T. P., \& Quinney, H. A. (2003). Effects of taper on endurance cycling capacity and single muscle fiber properties. Medicine and Science in Sports and Exercise, 35(11), 1875-1881. https://doi.org/10.1249/01.MSS.0000093617.28237.20

Nuzzo, J. L., McBride, J. M., Cormie, P., \& McCaulley, G. O. (2008). Relationship Between Countermovement Jump Performance and Multijoint Isometric and Dynamic Tests of Strength. The Journal of Strength \& Conditioning Research, 22(3), 699.

Orvanová, E. (1990). Somatotypes of weight lifters. Journal of Sports Sciences, 8(2), 119-137. https://doi.org/10.1080/02640419008732139

Painter, K. B., Haff, G. G., Ramsey, M. W., McBride, J., Triplett, T., Sands, W. A., ... Stone, M. H. (2012). Strength gains: block versus daily undulating periodization weight training among track and field athletes. International Journal of Sports Physiology and Performance, 7(2), $161-169$. 
Pelka, M., Schneider, P., \& Kellmann, M. (2017). Development of pre- and post-match morning recovery-stress states during in-season weeks in elite youth football. Science and Medicine in Football, O(0), 1-6. https://doi.org/10.1080/24733938.2017.1384560

Phillips, S. K., Sanderson, A. G., Birch, K., Bruce, S. A., \& Woledge, R. C. (1996). Changes in maximal voluntary force of human adductor pollicis muscle during the menstrual cycle. The Journal of Physiology, 496(Pt 2), 551-557. Retrieved from https://www.ncbi.nlm.nih.gov/pmc/articles/PMC1160898/

Pistilli, E. E., Kaminsky, D. E., Totten, L. M., \& Miller, D. R. (2008). Incorporating One Week of Planned Overreaching into the Training Program of Weightlifters. Strength \& Conditioning Journal, 30(6). Retrieved from http://journals.1ww.com/nscascj/Fulltext/2008/12000/Incorporating One Week of Planned Overreaching.5.aspx

Pritchard, H. J., Barnes, M. J., Stewart, R. J. C., Keogh, J. W. L., \& McGuigan, M. R. (2018). Short-Term Training Cessation as a Method of Tapering to Improve Maximal Strength. The Journal of Strength \& Conditioning Research, 32(2), 458.

https://doi.org/10.1519/JSC.0000000000001803

Pritchard, H. J., Tod, D. A., Barnes, M. J., Keogh, J. W., \& McGuigan, M. R. (2016). Tapering Practices of New Zealand's Elite Raw Powerlifters. Journal of Strength and Conditioning Research, 30(7), 1796-1804. https://doi.org/10.1519/JSC.0000000000001292

Pritchard, H., Keogh, J., Barnes, M., \& McGuigan, M. (2015). Effects and Mechanisms of Tapering in Maximizing Muscular Strength. Strength \& Conditioning Journal, 37(2). Retrieved from http://journals.lww.com/nscascj/Fulltext/2015/04000/Effects_and Mechanisms_of Tapering in Maximizing.3.aspx 
Pyne, D. B., Mujika, I., \& Reilly, T. (2009). Peaking for optimal performance: Research limitations and future directions. Journal of Sports Sciences, 27(3), 195-202. https://doi.org/10.1080/02640410802509136

Raeder, C., Wiewelhove, T., Schneider, C., Döwelling, A., Kellmann, M., Meyer, T., ... Ferrauti, A. (2017). Effects of active recovery on muscle function following high-intensity training sessions in elite olympic weightlifters. Advances in Skeletal Muscle Function Assessment, $1(1), 3-12$.

Raglin, J. S., Koceja, D. M., Stager, J. M., \& Harms, C. A. (1996). Mood, neuromuscular function, and performance during training in female swimmers. Medicine and Science in Sports and Exercise, 28(3), 372-377.

Rohleder, N., Beulen, S. E., Chen, E., Wolf, J. M., \& Kirschbaum, C. (2007). Stress on the dance floor: the cortisol stress response to social-evaluative threat in competitive ballroom dancers. Personality \& Social Psychology Bulletin, 33(1), 69-84. https://doi.org/10.1177/0146167206293986

Sale, D. G. (1988). Neural adaptation to resistance training. Medicine and Science in Sports and Exercise, 20(5 Suppl), S135-145.

Santos, E. J. A. M., \& Janeira, M. A. A. S. (2009). Effects of reduced training and detraining on upper and lower body explosive strength in adolescent male basketball players. Journal of Strength and Conditioning Research, 23(6), 1737-1744.

https://doi.org/10.1519/JSC.0b013e3181b3dc9d

Sarwar, R., Niclos, B. B., \& Rutherford, O. M. (1996). Changes in muscle strength, relaxation rate and fatiguability during the human menstrual cycle. The Journal of Physiology, 493(Pt 1), 267-272. Retrieved from https://www.ncbi.nlm.nih.gov/pmc/articles/PMC1158967/ 
Scanlon, T. C., Fragala, M. S., Stout, J. R., Emerson, N. S., Beyer, K. S., Oliveira, L. P., \& Hoffman, J. R. (2014). Muscle architecture and strength: adaptations to short-term resistance training in older adults. Muscle \& Nerve, 49(4), 584-592. https://doi.org/10.1002/mus.23969

Secomb, J. L., Nimphius, S., Farley, O. R. L., Lundgren, L. E., Tran, T. T., \& Sheppard, J. M. (2015). Relationships Between Lower-Body Muscle Structure and, Lower-Body Strength, Explosiveness and Eccentric Leg Stiffness in Adolescent Athletes. Journal of Sports Science \& Medicine, 14(4), 691-697. Retrieved from http://www.ncbi.nlm.nih.gov/pmc/articles/PMC4657409/

Smith, L. L. (2000). Cytokine hypothesis of overtraining: a physiological adaptation to excessive stress? Medicine and Science in Sports and Exercise, 32(2), 317-331.

Siart, B., Nimmerichter, A., Vidotto, C., \& Wallner, B. (2017). Status, Stress and Performance in Track and Field Athletes during the European Games in Baku (Azerbaijan). Scientific Reports, 7(1), 6076. https://doi.org/10.1038/s41598-017-06461-z

Staron, R. S., Malicky, E. S., Leonardi, M. J., Falkel, J. E., Hagerman, F. C., \& Dudley, G. A. (1990). Muscle hypertrophy and fast fiber type conversions in heavy resistance-trained women. European Journal of Applied Physiology and Occupational Physiology, 60(1), 7179.

Stebbings, G. K. (2015, January). Genetics of skeletal muscle strength and its determinants in healthy, untrained males (doctoral). Manchester Metropolitan University. Retrieved from https://e-space.mmu.ac.uk/580126/

Stewart, L. K., Flynn, M. G., Campbell, W. W., Craig, B. A., Robinson, J. P., Timmerman, K. L., ... Talbert, E. (2007). The influence of exercise training on inflammatory cytokines and 
C-reactive protein. Medicine and Science in Sports and Exercise, 39(10), 1714-1719. https://doi.org/10.1249/mss.0b013e31811ece1c

Stoessel, L., Stone, M. H., Keith, R., Marple, D., \& Johnson, R. (1991). Selected Physiological, Psychological and Performance Characteristics of National-Caliber United States Women Weightlifters. The Journal of Strength \& Conditioning Research, 5(2), 87. Retrieved from https://journals.lww.com/nscajscr/abstract/1991/05000/selected physiological, psychological and.7.aspx

Stone, M. H., Fry, A. C., Thrush, J. T., Fleck, S. J., Kraemer, W. J., Kearney, J. T., \& Marsit, J. (1993). Overtraining in weightlifters. Presented at the Weightlifting Symposium, Ancient Olympia, Greece.

Stone, M. H., O’Bryant, H., \& Garhammer, J. (1981). A hypothetical model for strength training. The Journal of Sports Medicine and Physical Fitness, 21(4), 342-351.

Stone, M., Plisk, S., \& Collins, D. (2002). Training principles: evaluation of modes and methods of resistance training--a coaching perspective. Sports Biomechanics, 1(1), 79-103. https://doi.org/10.1080/14763140208522788

Stone, M.H., Josey, J., Hunter, G., Kearney, J. T., Fry, A. C., Kraemer, W. J., ... Haff, G. G. (1996). Different taper lengths: effects of weightlifting performance. In Overtraining and Overreaching in Sport Conference (p. 59). University of Memphis, TN.

Stone, Michael H. (1993). Explosive Exercise and Training. Journal of Strength and Conditioning Research, 15(7), 7-15.

Stone, Michael H., \& Kenton B. Kirksey. (2000). Physiology of Weight Lifting. In Exercise and Sport Science (1st ed., pp. 941-954). Philadelphia, PA USA: Lippincott Williams \& Wilkins. 
Stone, Michael H., Pierce E, K. C., Sands, W. A., \& Stone, M. E. (2006). Weightlifting: Program Design. Strength and Conditioning Journal; Lawrence, 28(2), 10-17. Retrieved from https://search.proquest.com/docview/212589029/abstract/B36BD8EC3D784A77PQ/1

Stone, Michael H., Pierce, K. C., Sands, W. A., \& Stone, M. E. (2006). Weightlifting: A Brief Overview. Strength \& Conditioning Journal, 28(1), 50.

Stone, Michael H., Sands, W. A., Pierce, K. C., Carlock, J., Cardinale, M., \& Newton, R. U. (2005). Relationship of maximum strength to weightlifting performance. Medicine and Science in Sports and Exercise, 37(6), 1037-1043.

Stone, Michael H., Stone, M., \& Sands, W. A. (2007). Principles and Practice of Resistance Training. Human Kinetics.

Storey, A., \& Smith, H. K. (2012). Unique aspects of competitive weightlifting: performance, training and physiology. Sports Medicine (Auckland, N.Z.), 42(9), 769-790. https://doi.org/10.2165/11633000-000000000-00000

Storey, A., Wong, S., Smith, H. K., \& Marshall, P. (2012). Divergent muscle functional and architectural responses to two successive high intensity resistance exercise sessions in competitive weightlifters and resistance trained adults. European Journal of Applied Physiology, 112(10), 3629-3639. https://doi.org/10.1007/s00421-012-2346-4

Takano, B. (1989). Bulgarian Training Program: The 1989 NSCA Bulgaria-U.S.S.R. study tour - the organization of the Bulgarian national weightlifting program. Strength \& Conditioning Journal, 11(5), 38. Retrieved from https://journals.1ww.com/nscascj/Citation/1989/10000/BULGARIAN TRAINING PROGRAM The 1989 NSCA.4.asp $\underline{\mathrm{X}}$ 
Thomas, L., \& Busso, T. (2005). A theoretical study of taper characteristics to optimize performance. Medicine and Science in Sports and Exercise, 37(9), 1615-1621.

Tønnessen, E., Sylta, Ø., Haugen, T. A., Hem, E., Svendsen, I. S., \& Seiler, S. (2014). The road to gold: training and peaking characteristics in the year prior to a gold medal endurance performance. PloS One, 9(7), e101796. https://doi.org/10.1371/journal.pone.0101796

Trappe, S., Godard, M., Gallagher, P., Carroll, C., Rowden, G., \& Porter, D. (2001). Resistance training improves single muscle fiber contractile function in older women. American Journal of Physiology. Cell Physiology, 281(2), C398-406.

Travis, S. K., Goodin, J. G., Suarez, D. G., \& Bazyler, C. D. (2017). Identifying a surrogate measure of weightlifting performance. Presented at the 12th Annual Coaching and Sport Science College, Johnson City, TN.

Trinity, J. D., Pahnke, M. D., Sterkel, J. A., \& Coyle, E. F. (2008). Maximal power and performance during a swim taper. International Journal of Sports Medicine, 29(6), 500-506. https://doi.org/10.1055/s-2007-965784

Trinity, Joel D., Pahnke, M. D., Reese, E. C., \& Coyle, E. F. (2006). Maximal mechanical power during a taper in elite swimmers. Medicine and Science in Sports and Exercise, 38(9), 16431649. https://doi.org/10.1249/01.mss.0000229104.39145.6b

Vacher, P., Nicolas, M., Martinent, G., \& Mourot, L. (2017). Changes of Swimmers' Emotional States during the Preparation of National Championship: Do Recovery-Stress States Matter? Frontiers in Psychology, 8. https://doi.org/10.3389/fpsyg.2017.01043

Viru, A. A., \& Viru, M. (2001). Biochemical monitoring of sport training. Champaign, IL : Human Kinetics. Retrieved from http://trove.nla.gov.au/version/44897223 
Vizcaya, F. J., Viana, O., del Olmo, M. F., \& Acero, R. M. (2009). Could the deep squat jump predict weightlifting performance? Journal of Strength and Conditioning Research, 23(3), 729-734. https://doi.org/10.1519/JSC.0b013e3181a04dc3

Walker, S., Häkkinen, K., Haff, G. G., Blazevich, A. J., \& Newton, R. U. (2017). Acute elevations in serum hormones are attenuated after chronic training with traditional isoinertial but not accentuated eccentric loads in strength-trained men. Physiological Reports, 5(7). https://doi.org/10.14814/phy2.13241

Wilson, J. M., \& Wilson, G. J. (2008). A Practical Approach to the Taper. Strength and Conditioning Journal, 30(2), 10. Retrieved from https://dialnet.unirioja.es/servlet/articulo?codigo $=2780258$

Winwood, P. W., Dudson, M. K., Wilson, D., Mclaren-Harrison, J., Redjkins, V., Pritchard, H. J., \& Keogh, J. W. L. (2018). The Tapering Practices of Strongman Athletes. The Journal of Strength \& Conditioning Research, Publish Ahead of Print. https://doi.org/10.1519/JSC.0000000000002453

Wu, C.-L., Hung, W., Wang, S.-Y., \& Chang, C.-K. (2008). Hormonal Responses in Heavy Training and Recovery Periods in an Elite Male Weightlifter. Journal of Sports Science \& Medicine, 7(4), 560-561. Retrieved from http://www.ncbi.nlm.nih.gov/pmc/articles/PMC3761924/

Zaras, N. D., Stasinaki, A.-N. E., Krase, A. A., Methenitis, S. K., Karampatsos, G. P., Georgiadis, G. V., ... Terzis, G. D. (2014). Effects of tapering with light vs. heavy loads on track and field throwing performance. Journal of Strength and Conditioning Research, 28(12), 3484-3495. https://doi.org/10.1519/JSC.0000000000000566 
Zaras, N. D., Stasinaki, A.-N. E., Methenitis, S. K., Krase, A. A., Karampatsos, G. P., Georgiadis, G. V., ... Terzis, G. D. (2016). Rate of Force Development, Muscle Architecture, and Performance in Young Competitive Track and Field Throwers. Journal of Strength and Conditioning Research, 30(1), 81-92. https://doi.org/10.1519/JSC.0000000000001048

Zatsiorsky, V. M. (1992). International Perspective: Intensity of Strength Training Facts and TheoryRussian and Eastern European Approach. Strength \& Conditioning Journal, 14(5), 46. Retrieved from https://journals.1ww.com/nscascj/Citation/1992/10000/International_Perspective Intensity of Strength.11.aspx 


\section{APPENDICES \\ APPENDIX A: Potential Conflict of Interest Form}

Potential Conflict of Interest Form for Study Staff

The thresholds of ownership described below apply to the aggregate ownership of an individual study staff member, his/her spouse, domestic partner and dependent children (e.g., if a study staff member, his/her spouse, domestic partner and dependent children own together $\$ 5,000$ or $5 \%$ worth of equities in the sponsor, it should be reported below).

Please choose the applicable box(s).

$\square$ financial interest in the research with value that can not be readily determined

financial interest in the research with value that exceeds the specified monetary threshold $(\$ 5,000)$ or $5 \%$ ownership

has received or will receive compensation whose value could be affected by the study outcome.

$\square$ has a proprietary interest in the research included but not limited to, a patent, trademark, copyright or licensing agreement, or the right to receive royalties from product commercialization.

$\square$ has received payments from the sponsor that exceed the specified monetary threshold $(\$ 5,000)$ in the past year.

$\square$ is executive or director of the agency or company sponsoring the research

Equity interest of less than $\$ 5,000$ when that value could not be determined through reference to public prices or other reasonable measures of fair market value

None of the above

If your answer is NOT "none of the above", you must follow the additional reporting/management requirements in IRB Policy $17 a$.

Attestation:

I agree to submit a revised Conflict of Interest Form to the PI and IRB within ten days of any change from previous disclosures.

Spencer Kyle Travis

Typed/Printed Name

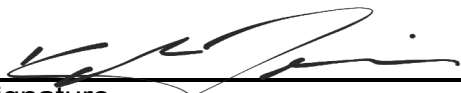

Signature

Protocol(s)

Revision date 11/23/15 


\section{APPENDIX B: Dietary Food Log}

\section{DIETARY LOG}

1. Use the Dietary Record Forms provided to record everything you eat or drink for each day of this study.

2. Indicate the name of the FOOD ITEM, the AMOUNT eaten, how it was PREPARED (fried, boiled, etc.), and the TIME the food was eaten. If the item was a brand name product, please include the name. Try to be accurate about the amounts eaten. Measuring with measuring cups and spoons is best, but if you must make estimates, use the following guidelines: Fist is about 1 cup

Tip of Thumb is about 1 teaspoon

Palm of the hand is about 3 ounces of meat (about the size of a deck of cards) Tip of

Thumb is about 1 ounce of cheese

3. Try to eat what you normally eat and record everything. The project will only be useful if you are HONEST about what you eat. The information you provide is confidential.

4. MILK: Indicate whether milk is whole, low fat (1 or $2 \%)$, or skim. Include flavoring if one is used.

5. VEGETABLES and FRUITS: One average serving of cooked or canned fruits and vegetables is about a half cup. Fresh whole fruits and vegetables should be listed as small, medium, or large. Be sure to indicate if sugar or syrup is added to fruit and list if any margarine, butter, cheese sauce, or cream sauce is added to vegetables. When recording salad, list items comprising the salad separately and be sure to include salad dressing used.

6. EGGS: Indicate method of preparation (scrambled, fried, poaches, etc.) and number eaten.

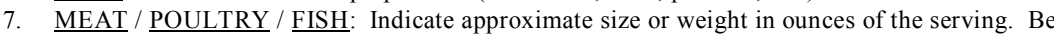
sure to include any gravy, sauce, or breading added.

8. CHEESE: Indicate kind, number of ounces or slices, and whether it is made from whole milk, part skim, or is low calorie.

9. CEREAL: Specify kind, whether cooked or dry, and measure in terms or cups or ounces. Remember that consuming $8 \mathrm{oz}$. of cereal is not the same as consuming one cup of cereal. 1 cup of cereal generally weighs about 1 ounce.

10. BREAD and ROLLS: Specify kind (whole wheat, enriched wheat, rye, etc.) and number of slices.

11. BEVERAGES: Include every item you drink excluding water. Be sure to record cream and sugar used in tea and coffee, whether juices are sweetened or unsweetened and whether soft drinks are diet or regular.

12. FATS: Remember to record all butter, margarine, oil, and other fats used in cooking or on food.

13. MIXED DISHES / CASSEROLES: List the main ingredients and approximate amount of each ingredient to the best of your ability.

14. ALCOHOL: Be honest. Record amounts in ounces. Specify with "light" or "regular" beer.

\section{DIETARY RECORD FORM}

Day of the Week:

Date: 


\begin{tabular}{|c|c|c|}
\hline FOOD ITEM & AMOUNT & TIME \\
\hline & & \\
\hline & & \\
\hline & & \\
\hline & & \\
\hline & & \\
\hline & & \\
\hline & & \\
\hline & & \\
\hline & & \\
\hline & & \\
\hline & & \\
\hline & & \\
\hline & & \\
\hline & & \\
\hline & & \\
\hline & & \\
\hline & & \\
\hline & & \\
\hline & & \\
\hline & & \\
\hline & & \\
\hline & & \\
\hline & & \\
\hline & & \\
\hline & & \\
\hline & & \\
\hline & & \\
\hline
\end{tabular}

Express approximate measures in cups $(\mathrm{C})$, tablespoons $(\mathrm{T})$, teaspoons $(\mathrm{t})$, grams $(\mathrm{g})$, ounces $(\mathrm{oz}$.$) ,$ pieces, etc. 


\section{APPENDIX C: Sample of Short Recovery-Stress Scale}

\section{Short Recovery Scale}

Below you find a list of expressions that describe different aspects of your current state of recovery. Rate how you feel right now in relation to your best ever recovery state.

\section{Physical Performance}

\section{Capability}

e.g.

strong,

physically capable,

energetic,

full of power

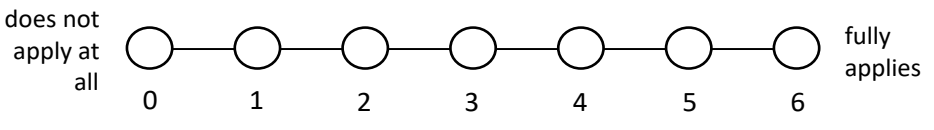

Mental Performance

\section{Capability}

e.g.

attentive,

receptive,

concentrated,

mentally alert

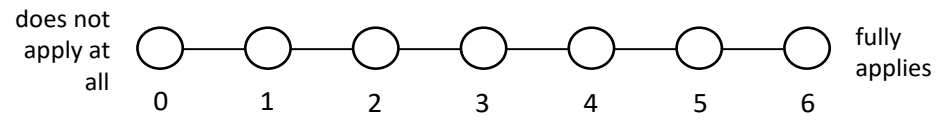

\section{Emotional Balance}

e.g.

satisfied,

balanced,

in a good mood,

having everything under control

stable,

pleased

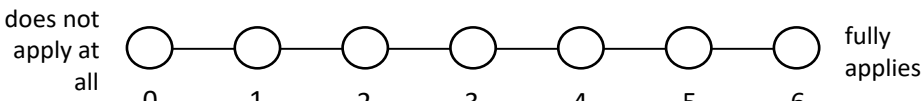

does not apply at all applies

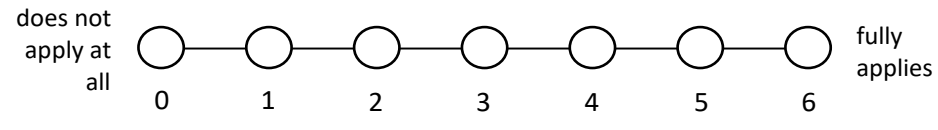

\section{Overall Recovery}

e.g.

recovered,

rested,

muscle relaxation,

physically relaxed 


\section{Short Stress Scale}

Below you find a list of expressions that describe different aspects of your current state of stress. Rate how you feel right now in relation to your highest ever stress state.

\section{Muscular Stress}

e.g.

muscle exhaustion

muscle fatigue,

muscle soreness,

muscle stiffness

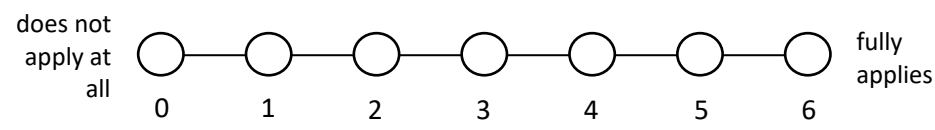

\section{Lack of Activation}

e.g

unmotivated,

sluggish,

unenthusiastic,

lacking energy

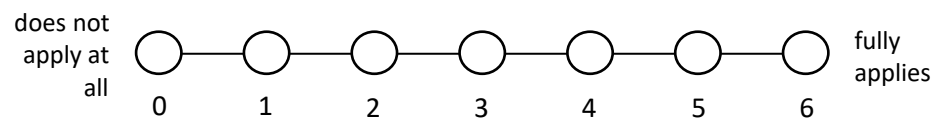

Negative Emotional State

e.g.

feeling down,

stressed,

annoyed

short-tempered

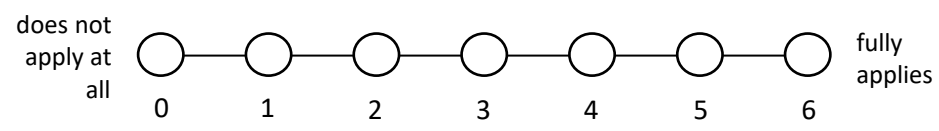

\section{Overall Stress}

e.g.

tired,

worn-out,

overloaded,

physically exhausted

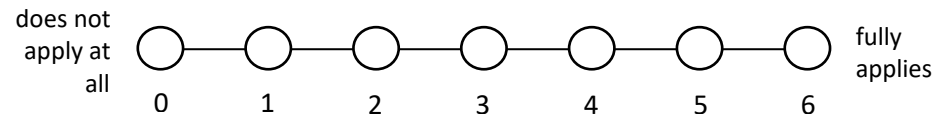


APPENDIX D: Ultrasonography Imaging for Muscle Thickness

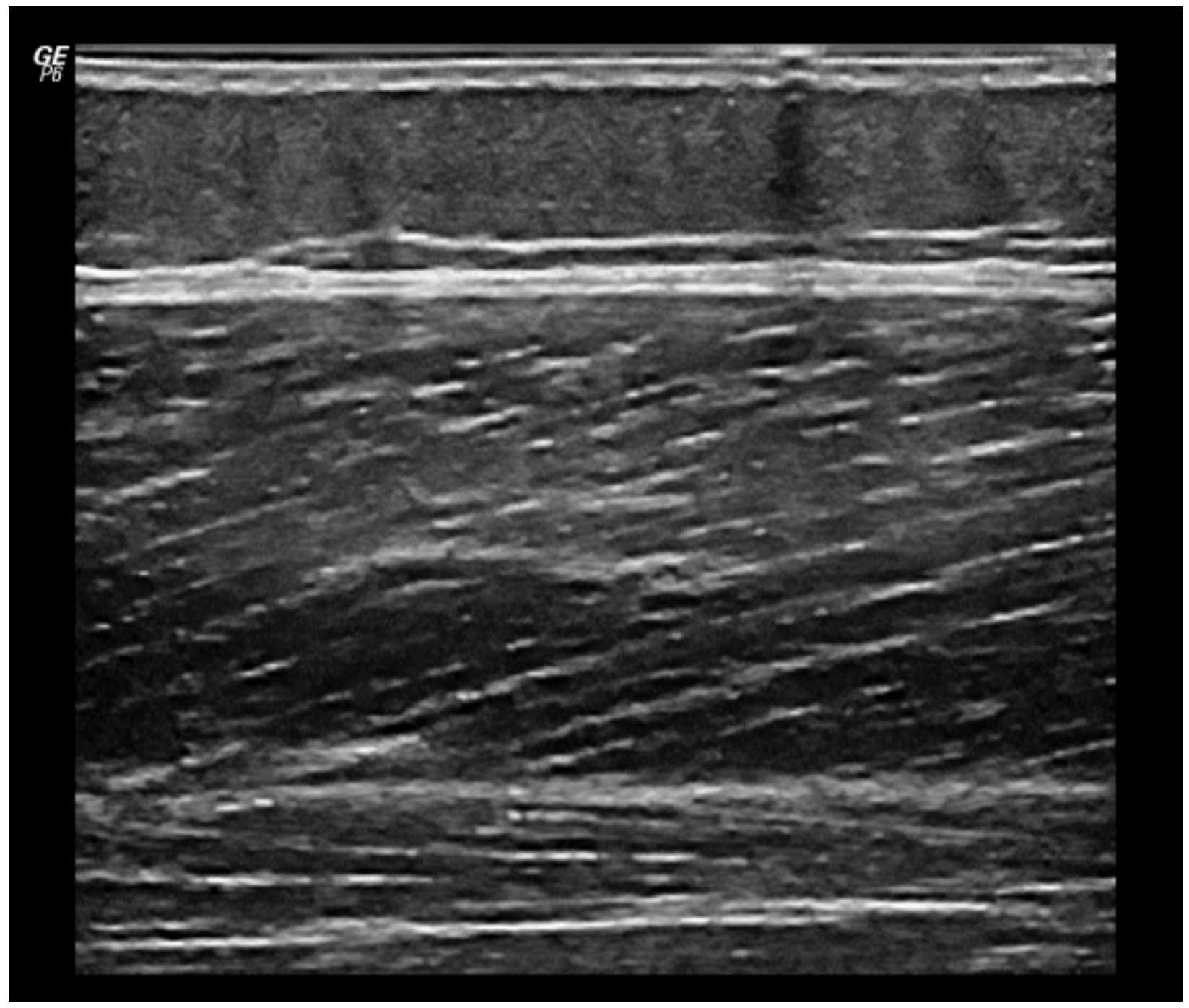


APPENDIX E: Ultrasonography Imaging for Muscle Cross-Sectional Area

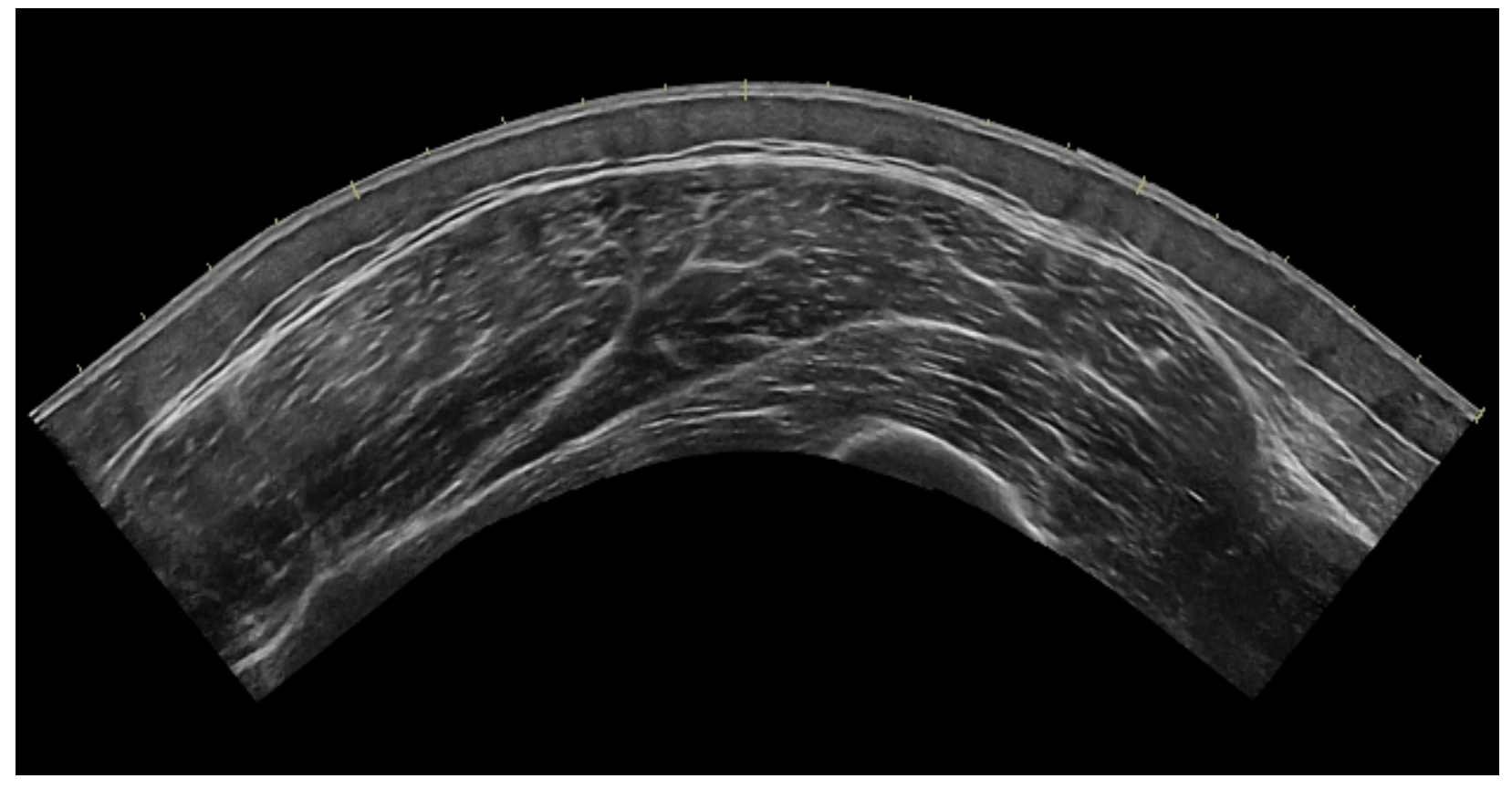


APPENDIX F: Sample Data of Squat Jump Performance Collection Program

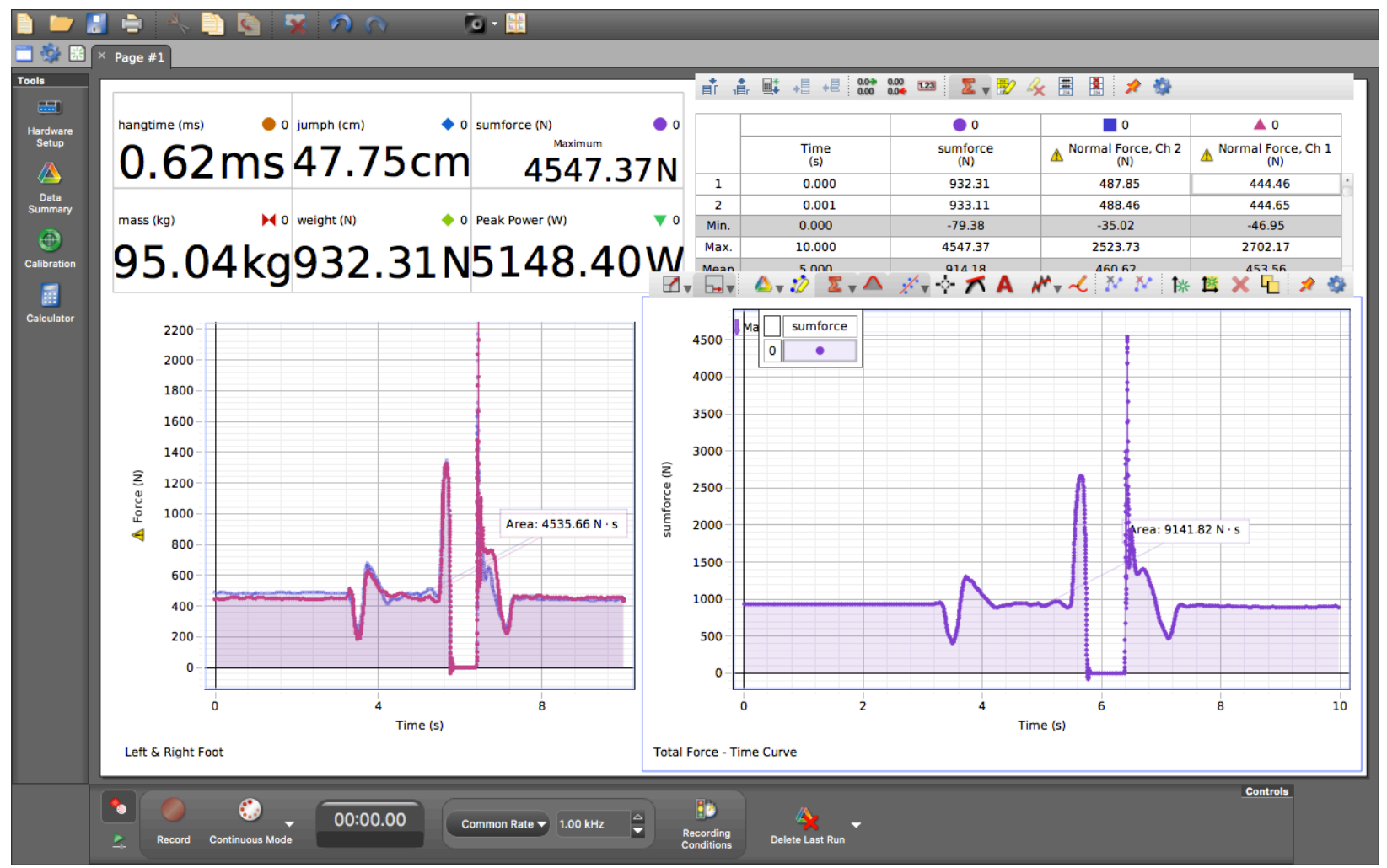




\section{APPENDIX G: Sample Data of Squat Jump Performance Analysis Program}

\begin{tabular}{|c|c|c|c|c|c|c|c|c|c|c|c|c|c|c|c|c|c|}
\hline Testing date & Jump type & Note & File Name & Jump \# & Bar (kg) & Offset & Threshold & SW (N) & $\mathrm{JH}-\mathrm{FT}(\mathrm{m})$ & $\mathrm{JH}-\mathrm{NI}(\mathrm{m})$ & $\mathrm{NI}\left(\mathrm{N}^{\bullet} \mathrm{s}\right)$ & $\mathrm{PF}(\mathrm{N})$ & $\mathrm{PV}(\mathrm{m} / \mathrm{s})$ & PP (W) & F $@ P P(N)$ & $\mathrm{V} @ \mathrm{PP}(\mathrm{m} / \mathrm{s})$ & RFD (N/s) \\
\hline $10 / 14 / 17$ & SJ & & W3.3.1 & 1 & 0 & 17.748 & 7.5 & 546.59 & 0.316 & 0.320 & 139.58 & 1394.63 & 2.59 & 3016.49 & 1315.84 & 2.29 & 3656.686 \\
\hline $10 / 14 / 17$ & $7 \mathrm{SJ}$ & & W3.3.1 & 2 & 0 & 18.938 & 7.5 & 543.40 & 0.324 & 0.323 & 139.41 & 1400.47 & 2.60 & 3002.59 & 1306.85 & 2.30 & 4228.137 \\
\hline $10 / 14 / 17$ & SJ & & W3.3.1 & 3 & 0 & 19.043 & 7.5 & 544.79 & 0.328 & 0.330 & 141.25 & 1379.04 & 2.62 & 3010.04 & 1291.87 & 2.33 & 3699.053 \\
\hline $10 / 14 / 17$ & SJ & & W3.3.1 & 4 & 0 & 20.042 & 7.5 & 545.55 & 0.320 & 0.323 & 140.01 & 1342.00 & 2.60 & 2952.06 & 1275.54 & 2.31 & 2875.118 \\
\hline $10 / 14 / 17$ & SJ & & W3.3.1 & 5 & 0 & 16.179 & 7.5 & 850.10 & 0.440 & 0.432 & 252.21 & 2354.54 & 2.99 & 5937.75 & 2215.17 & 2.68 & 5122.832 \\
\hline $10 / 14 / 17$ & $\mathrm{SJ}$ & & W3.3.1 & 6 & 0 & 17.406 & 7.5 & 841.97 & 0.467 & 0.492 & 266.57 & 2414.70 & 3.18 & 6496.92 & 2296.08 & 2.83 & 5243.571 \\
\hline $10 / 14 / 17$ & SJ & & W3.3.1 & 7 & 0 & 17.566 & 7.5 & 837.58 & 0.473 & 0.500 & 267.48 & 2384.48 & 3.23 & 6536.19 & 2269.73 & 2.88 & 4707.362 \\
\hline $10 / 14 / 17$ & SJ & & W3.3.1 & 8 & 0 & 16.318 & 7.5 & 927.23 & 0.529 & 0.539 & 294.01 & 2698.30 & 3.19 & 6970.33 & 2495.95 & 2.79 & 8556.051 \\
\hline $10 / 14 / 17$ & SJ & & W3.3.1 & 9 & 0 & 18.63 & 7.5 & 926.02 & 0.534 & 0.547 & 300.72 & 2684.14 & 3.27 & 7086.86 & 2483.46 & 2.85 & 8843.333 \\
\hline $10 / 14 / 17$ & SJ & & W3.3.1 & 10 & 0 & 17.015 & 7.5 & 930.54 & 0.524 & 0.510 & 297.13 & 2730.95 & 3.21 & 7064.65 & 2514.06 & 2.81 & 8902.562 \\
\hline
\end{tabular}




\section{VITA}

\section{SPENCER KYLE TRAVIS}

Education:

Professional Experience:
M.S. Sport Science and Coach Education, East Tennessee State University, Johnson City, TN, 2018

B.S. Kinesiology and Human Performance: Emphasis in Exercise Physiology, The University of Southern Mississippi, Hattiesburg, MS, 2016

Graduate Assistant, East Tennessee State University, Johnson City, TN, 2017-2018

Lead Sport Scientist, Head Strength \& Conditioning Coach, Men and Women's Tennis; Strength Coach, Para-Powerlifting, East Tennessee State University, Johnson City, TN, 2016-2018

Committee Member, Graduate Professional Student Organization, Graduate Representative for Applied Sport Science, Sport Physiology \& Sport Performance Programs, East Tennessee State University, Johnson City, TN, 2016-2018

Tuition Scholar, Graduate Professional Student Organization, Director of Operations, East Tennessee State University, Johnson City, TN, 2017

Intern, Olympic Training Site, East Tennessee State University, Johnson City, TN, 2016

Classes Taught:

Sport Conditioning and Training, Guest Lecturer, East Tennessee State University, Johnson City, TN, Fall 2016

Biomechanics Laboratory; Development of Strength \& Conditioning Programs; Physiological Principles of Resistance Training, The University of Southern Mississippi, Hattiesburg, MS, 2014-2016

Publications/Abstracts: $\quad$ Travis, S.K., Mizuguchi, S., Stone, M.H., Sands, W.A., Bazyler, C.D. Preparing for national weightlifting championship: a case study. Presenting at the National Strength and Conditioning Association National Conference, Indianapolis, IN 2018.

Travis, S.K., Goodin, J.R., Beckham, G.K., Bazyler, C.D. (In Press, May 2018) Identifying an indicative performance measure 
to monitor weightlifting performance in competitive male and female weightlifters. Sports.

Travis, S.K., Goodin, J.R., Bazyler, C.D. Quantifying performance characteristics of an international level male weightlifter. Presented at the Annual Meeting of the Southeast Chapter of the American College of Sports Medicine, Chattanooga, TN 2018.

Travis, S.K., Goodin, J.R., Suarez, D., Bazyler, C.D. Identifying a surrogate measure of weightlifting performance. Presented at $12^{\text {th }}$ Annual Coaching and Sport Science College Symposium, Johnson City, TN 2017.

Travis, S.K. \& Walters, J. Time-series forecasting: a theoretical model for predicting performance potential. Presented at $12^{\text {th }}$ Annual Coaching and Sport Science College Symposium, Johnson City, TN 2017.

Coniglio, C.L., Travis, S.K., Gentles, J.A. The relationship between accelerometry and total distance measured with GPS in women's college soccer players. Presented at $12^{\text {th }}$ Annual Coaching and Sport Science College Symposium, Johnson City, TN 2017.

Goodin, J.R., Bernards, J.R., Travis, S.K., Wines, M., Smith, T., Stone, M.H. Intraset variability of concentric mean velocity in the back squat. Presented at $11^{\text {th }}$ Annual Coaching and Sport Science College Symposium, Johnson City, TN 2016.

Honors and Awards: $\quad$ National Strength and Conditioning Association - Certified Strength and Conditioning Specialist, 2016 - Present

2017 SoCon Conference Championship, ETSU Men's Tennis, Sport Scientist and Strength Coach

2018 SoCon Conference Championship, ETSU Men's Tennis, Sport Scientist and Strength Coach

Nominated for Top 10 Exceptional Abstracts, National Strength and Conditioning Association National Conference, Indianapolis, IN 2018. 